\title{
Dynamics of an adaptive randomly reinforced urn
}

\author{
GIACOMO ALETTI ${ }^{1, *}$, ANDREA GHIGLIETTI ${ }^{1, * *}$ and \\ ANAND N. VIDYASHANKAR ${ }^{2}$ \\ ${ }^{1}$ ADAMSS Center, Università degli Studi di Milano, Milan, Italy. \\ E-mail:*'giacomo.aletti@unimi.it; ${ }^{* *}$ andrea.ghiglietti@unimi.it \\ ${ }^{2}$ Department of Statistics, George Mason University, Fairfax, VA, USA. E-mail: avidyash@gmu.edu
}

Adaptive randomly reinforced urn (ARRU) is a two-color urn model where the updating process is defined by a sequence of non-negative random vectors $\left\{\left(D_{1, n}, D_{2, n}\right) ; n \geq 1\right\}$ and randomly evolving thresholds which utilize accruing statistical information for the updates. Let $m_{1}=E\left[D_{1, n}\right]$ and $m_{2}=E\left[D_{2, n}\right]$. In this paper, we undertake a detailed study of the dynamics of the ARRU model. First, for the case $m_{1} \neq m_{2}$, we establish $L_{1}$ bounds on the increments of the urn proportion, that is, the proportion of ball colors in the urn, at fixed and increasing times under very weak assumptions on the random threshold sequences. As a consequence, we deduce weak consistency of the evolving urn proportions. Second, under slightly stronger conditions, we establish the strong consistency of the urn proportions for all finite values of $m_{1}$ and $m_{2}$. Specifically, we show that when $m_{1}=m_{2}$, the proportion converges to a non-degenerate random variable. Third, we establish the asymptotic distribution, after appropriate centering and scaling, for the proportion of sampled ball colors and urn proportions for the case $m_{1}=m_{2}$. In the process, we resolve the issue concerning the asymptotic distribution of the proportion of sampled ball colors for a randomly reinforced urn (RRU). To address the technical issues, we establish results on the harmonic moments of the total number of balls in the urn at different times under very weak conditions, which is of independent interest.

Keywords: central limit theorems; crossing times; generalized Pólya urn; harmonic moments; reinforced processes; strong and weak consistency

\section{Introduction}

In recent years, randomly reinforced urn (RRU) has been investigated in statistical and probability literature as a model for clinical trial design, computer experiments, and in the context of vertex reinforced random walk (see Hu and Rosenberger [18], Mahmoud [20], Pemantle and Volkov [23]). Introduction of accruing information in designing the reinforcement mechanism leads to an adaptive version of an RRU model, which we refer to as an adaptive randomly reinforced urn (ARRU). In this paper, we study the properties concerning the urn proportions and the proportion of sampled ball colors of an ARRU. We now turn to a precise description of the ARRU.

A randomly reinforced urn (RRU) model (see Muliere, Paganoni and Secchi [22]) is characterized by a pair $\left(Y_{1, n}, Y_{2, n}\right)$ of real random variables representing the number of balls of two colors, red and white. The process is described as follows: at time $n=0$, the process starts with $\left(y_{1,0}, y_{2,0}\right)$ balls. A ball is drawn at random. If the color is red, the ball is returned to the urn 
along with the random numbers $D_{1,1}$ of red balls; otherwise, the ball is returned to the urn along with the random numbers $D_{2,1}$ of white balls. Let $Y_{1,1}=y_{1,0}+D_{1,1}$ and $Y_{2,1}=y_{2,0}$ denote the urn composition when the sampled ball is red; similarly, let $Y_{1,1}=y_{1,0}$ and $Y_{2,1}=y_{2,0}+D_{2,1}$ denote the urn composition when the sampled ball is white. The process is repeated yielding the collection $\left\{\left(Y_{1, n}, Y_{2, n}\right) ; n \geq 1\right\}$. The quantities $\left\{D_{1, n} ; n \geq 1\right\}$ and $\left\{D_{2, n} ; n \geq 1\right\}$ are independent collections of independent and identically distributed (i.i.d.) non-negative random variables.

The urn model can be also described using its replacement matrix $D_{n}$, where $\left[D_{i j, n}\right]$ indicates the number of balls of color $j$ that are replaced in the urn when a balls of color $i$ is sampled. In the RRU model, since the off-diagonal elements are 0 , we simplify the notation $D_{i i, n}$ to $D_{i, n}$. Hence, the RRU model is characterized by the replacement matrix

$$
\mathbf{D}_{n}=\left[\begin{array}{cc}
D_{1, n} & 0 \\
0 & D_{2, n}
\end{array}\right]
$$

Let $m_{1}:=\boldsymbol{E}\left[D_{1, n}\right]$ and $m_{2}:=\boldsymbol{E}\left[D_{2, n}\right]$.

Since the replacement matrix in (1.1) is diagonal, the RRU model is not a particular case of class of Generalized Pólya Urns (GPU) whose replacement matrix (or an almost sure limit of certain "conditional" replacement matrices) is assumed to be irreducible. For a review on the literature on GPUs, see, for instance, Athreya and Karlin [5], Smythe [24], Bai and Hu [6,7], Zhang, Hu and Cheung [25], Laruelle and Pagès [19], Aletti and Ghiglietti [1].

The asymptotic properties of the urn proportions in an RRU model were investigated by Durham, Flournoy and Li [12] for binary reinforcements, and extended to the continuous case by Muliere, Paganoni and Secchi [22], Aletti, May and Secchi [3]. Specifically, they established that

$$
Z_{n}=\frac{Y_{1, n}}{Y_{1, n}+Y_{2, n}} \stackrel{\text { a.s. }}{\rightarrow} \begin{cases}1 & \text { if } m_{1}>m_{2}, \\ Z_{\infty} & \text { if } m_{1}=m_{2}, \\ 0 & \text { if } m_{1}<m_{2},\end{cases}
$$

where $\stackrel{\text { a.s. }}{\rightarrow}$ stands for almost sure convergence and $Z_{\infty}$ is a random variable supported on $(0,1)$. The rate of convergence and the limit distribution of $Z_{n}$ when $m_{1} \neq m_{2}$ has been established in May and Flournoy [21]. For the case $m_{1}=m_{2}$, the properties of the distribution of $Z_{\infty}$ were studied in Durham, Flournoy and Li [12], Aletti, May and Secchi [3,4]. Specifically, the distribution of $Z_{\infty}$ when $D_{1, n}$ and $D_{2, n}$ are Bernoulli random variables with the same success probability, has been established in Durham, Flournoy and Li [12]. In the more general case where $D_{1, n}$ and $D_{2, n}$ have the same expectations, it has been proved in Aletti, May and Secchi [4] that the distribution of $Z_{\infty}$ is the unique continuous solution of a functional equation satisfying certain boundary conditions. Additionally, it is shown in Aletti, May and Secchi [3] that $\boldsymbol{P}\left(Z_{\infty}=x\right)=0$ for any $x \in[0,1]$. Denoting $\left\{\left(N_{1, n}, N_{2, n}\right) ; n \geq 1\right\}$ the number of balls of red and white colors sampled from the urn, one can deduce from (1.2) that $N_{1, n} / n$ converges to the same limit as $Z_{n}$.

Notice that for an RRU, the limit in (1.2) is always 1 or 0 in the case $m_{1} \neq m_{2}$. This asymptotic behavior can be very attractive in applications such as clinical trials, where the response-adaptive designs based on an RRU model achieve the ethical goal of assigning most subjects to a better performing treatment (see Durham, Flournoy and Li [12], Muliere, Paganoni and Secchi [22]). 
However, from an inferential perspective, it is common to target a specific value $\rho \in(0,1)$ (see $\mathrm{Hu}$ and Rosenberger [18] for applications in clinical trials). To perform clinical experiments with such a goal, a variant of RRU is needed. This was achieved in Aletti, Ghiglietti and Paganoni [2], where the modified randomly reinforced urn (MRRU) model was introduced. The MRRU model is an RRU with two fixed thresholds $0<\rho_{2} \leq \rho_{1}<1$, such that: (i) if a white ball is sampled and $Z_{n}<\rho_{2}$, no balls are replaced in urn, and (ii) if a red ball is sampled but $Z_{n}>\rho_{1}$, no balls are replaced in the urn. Hence, the replacement matrix (1.1) in this case becomes

$$
\mathbf{D}_{n}=\left[\begin{array}{cc}
D_{1, n} \cdot \mathbb{1}_{\left\{Z_{n-1} \leq \rho_{1}\right\}} & 0 \\
0 & D_{2, n} \cdot \mathbb{1}_{\left\{Z_{n-1} \geq \rho_{2}\right\}}
\end{array}\right] .
$$

A more precise description of the MRRU model is provided in Section 2, Remark 2.1.

The strong consistency of $Z_{n}$ in the case $m_{1} \neq m_{2}$ was established in Aletti, Ghiglietti and Paganoni [2], that is, they showed that

$$
Z_{n} \stackrel{\text { a.s. }}{\rightarrow} \begin{cases}\rho_{1} & \text { if } m_{1}>m_{2}, \\ \rho_{2} & \text { if } m_{1}<m_{2} .\end{cases}
$$

An efficient test based on the MRRU was implemented in Ghiglietti and Paganoni [15], while a second order result for $Z_{n}$ (again when $m_{1} \neq m_{2}$ ), namely the asymptotic distribution of $Z_{n}$ after appropriate centering and scaling, was derived in Ghiglietti and Paganoni [14]. We emphasize here that the rate of convergence in this case is not the usual $\sqrt{n}$ but $n$ and the limit distribution is not Gaussian.

In applications, especially in clinical trials (see Hu and Rosenberger [18]), $\rho_{1}$ and $\rho_{2}$ are unknown and depend on the parameters of the distributions of $D_{1,1}$ and $D_{2,1}$. Let $\mathcal{F}_{n-1}$ be the $\sigma$ algebra generated by the information up to time $n-1$ and let $\hat{\rho}_{1, n-1}$ and $\hat{\rho}_{2, n-1}$ be two random variables that are $\mathcal{F}_{n-1}$-measurable. Ghiglietti, Vidyashankar and Rosenberger [16] proposed an adaptive randomly reinforced urn model that uses accruing information to construct random thresholds $\hat{\rho}_{1, n-1}$ and $\hat{\rho}_{2, n-1}$ which converge a.s. to specified targets $\rho_{1}$ and $\rho_{2}$. Thus, using the replacement matrix

$$
\mathbf{D}_{n}=\left[\begin{array}{cc}
D_{1, n} \cdot \mathbb{1}_{\left\{Z_{n-1} \leq \hat{\rho}_{1, n-1}\right\}} & 0 \\
0 & D_{2, n} \cdot \mathbb{1}_{\left\{Z_{n-1} \geq \hat{\rho}_{2, n-1}\right\}}
\end{array}\right],
$$

an MRRU becomes an Adaptive Randomly Reinforced Urn (ARRU). It is worth mentioning here that the random thresholds $\hat{\rho}_{1, n-1}$ and $\hat{\rho}_{2, n-1}$ depend on the adaptive estimators of the parameters of the distributions of $D_{1,1}$ and $D_{2,1}$.

Ghiglietti, Vidyashankar and Rosenberger [16] studied the asymptotic properties of an ARRU when $m_{1} \neq m_{2}$ under various conditions on the rate of convergence of adaptive thresholds. Specifically, they established a strong consistency of (i) the proportion of sampled balls of each color and (ii) the urn proportions, under the assumption that the thresholds converge almost surely and that the limits of the thresholds are different from 0 and 1. Furthermore, they also establish the asymptotic normality for the number of sampled ball colors, under an exponential 
rate of convergence assumption on the adaptive thresholds and an additional condition that the thresholds are updated at exponential times. Additionally, they provided heuristics as to why the proportion of balls of each color in the urn (urn proportions) may not have a limiting Gaussian distribution, without further hypotheses.

In this manuscript, the first significant contribution concerns weak consistency results for the urn proportions when $m_{1} \neq m_{2}$ under the assumption that the threshold sequence $\left\{\hat{\rho}_{i, n}, n \geq 1\right\}$ converges in probability to $\rho_{i}$, for $i=1,2$. The hypothesis that the thresholds converge only in probability (and not a.s.) brings in subtle challenges which necessitate understanding the dynamics of the ARRU model. More precisely, our proofs involve obtaining $L_{1}$ bounds on (i) the increments of the distance $\Delta_{n}=\left|Z_{n}-\rho_{1}\right|$, viz. $\Delta_{n+1}-\Delta_{n}$ and (ii) the increments at linearly increasing times $\Delta_{n+n c}-\Delta_{n}$, where $c>0$. These results are then combined with a judicious choice of $c$ and using comparison arguments with a specifically designed RRU model, weak consistency is established. The above results are presented in Section 4; specifically, Theorem 4.2, Theorem 4.5, and Theorem 4.6. The proofs of these theorems rely on the estimates concerning the harmonic moments of the total number of balls in the urn. This result, of independent interest, is established in Theorem 4.1 where even the convergence of thresholds is not required.

The second significant contribution of this manuscript concerns the strong consistency of the urn proportions for all values of $m_{1}$ and $m_{2}$ under the assumption that the thresholds converge almost surely but without any restriction on their limiting values. As a consequence, we obtain the strong consistency of the proportion of sampled ball colors thus completing Corollary 2.1 in Ghiglietti, Vidyashankar and Rosenberger [16] for the case $m_{1}=m_{2}$. It is important to notice that in the case $m_{1}=m_{2}$, the urn proportion converges to a proper random variable $Z_{\infty}$ which is different from the case $m_{1} \neq m_{2}$. As a consequence, we obtain the strong consistency of the proportion of sampled balls of both the colors, thus completing Theorem 2.1 in Ghiglietti, Vidyashankar and Rosenberger [16] for the case $m_{1}=m_{2}$.

The third significant contribution concerns second order results for the urn proportions and the proportion of sampled ball colors for the case $m_{1}=m_{2}$. Specifically, we establish that, the quantities $\left(Z_{n}-Z_{\infty}\right)$ and $\left(n^{-1} N_{1, n}-Z_{\infty}\right)$ converge stably at the rate $\sqrt{n}$ to a distribution which is a continuous mixture of a centered Gaussian distribution and the distribution of $Z_{\infty}$. The proof involves decomposing $\left(n^{-1} N_{1, n}-Z_{\infty}\right)$ into a sum of two terms, one involving comparison of $n^{-1} N_{1, n}$ with the cumulative proportion of red balls up to time $n$ and the other involving the deviation of the cumulative proportion up to time $n$ from $Z_{\infty}$. The second term is then carefully investigated by invoking the Doob's decomposition theorem and some delicate estimates. In the process, we explicitly identify the variance of the conditional Gaussian distribution. This result also resolves a long-standing open problem in the well-investigated RRU model concerning the limiting distribution of the proportion of sampled ball colors for the case $m_{1}=m_{2}$. Additionally, the results also settle the open problem concerning the urn proportions and proportion of sampled ball colors for the MRRU model when $m_{1}=m_{2}$.

The rest of the paper is structured as follows: Section 2 contains the model, assumptions, and main results; Section 3 is concerned with preliminary estimates and results on the urn process. Sections 4 and 5 are concerned with the proofs of the consistency of the urn proportion and Section 6 is concerned with the proof of the limit distribution of the proportion of sampled balls of both the colors. 


\section{Model assumptions, notations, and main results}

We begin by describing our model precisely. Let $\xi_{1}=\left\{\xi_{1, n} ; n \geq 1\right\}$ and $\xi_{2}=\left\{\xi_{2, n} ; n \geq 1\right\}$ be two sequences of i.i.d. random variables. Without loss of generality (wlog), assume that the support $S$ of $\xi_{1, n}$ and $\xi_{2, n}$ to be the same. Additionally, let $\boldsymbol{U}=\left\{U_{n} ; n \geq 1\right\}$ denote a sequence of i.i.d. uniform random variables in $(0,1)$ independent of $\boldsymbol{\xi}_{1}$ and $\boldsymbol{\xi}_{2}$.

Consider an urn containing $y_{1,0}>0$ red balls and $y_{2,0}>0$ white balls, and define $y_{0}=y_{1,0}+$ $y_{2,0}$ and $z_{0}=y_{0}^{-1} y_{1,0}$. We note here that $y_{1,0}$ and $y_{2,0}$ may not assume integer values. At time $n=1$, a ball is drawn at random from the urn and its color is observed. Let the random variable $X_{1}$ be such that

$$
X_{1}= \begin{cases}1 & \text { if the extracted ball is red, } \\ 0 & \text { if the extracted ball is white }\end{cases}
$$

Then one can express $X_{1}=\mathbb{1}_{\left\{U_{1} \leq z_{0}\right\}}$. Note that $X_{1}$ is a Bernoulli random variable with parameter $z_{0}$ and is independent of $\boldsymbol{\xi}_{1}$ and $\boldsymbol{\xi}_{2}$.

Let $\hat{\rho}_{1,0}$ and $\hat{\rho}_{2,0}$ be two constants in $[0,1]$ and $\hat{\rho}_{1,0} \geq \hat{\rho}_{2,0}$. Let $u: S \rightarrow[a, b], 0<a \leq b<\infty$. (We mention here that it is possible to allow $a=0$. The case $a>0$ makes calculations transparent and hence we make this simplifying assumption throughout the manuscript. However, we add remarks on how to get rid of this assumption in various estimates that explicitly use $a>0$.) If $X_{1}=1$ and $z_{0} \leq \hat{\rho}_{1,0}$, we return the extracted ball to the urn together with $D_{1,1}=u\left(\xi_{1,1}\right)$ new red balls. While, if $X_{1}=0$ and $z_{0} \geq \hat{\rho}_{2,0}$, we return it to the urn together with $D_{2,1}=u\left(\xi_{2,1}\right)$ new white balls. If $X_{1}=1$ and $z_{0}>\hat{\rho}_{1,0}$, or if $X_{1}=0$ and $z_{0}<\hat{\rho}_{2,0}$, the urn composition is not modified. To ease notations, we set $w_{1,0}=\mathbb{1}_{\left\{z_{0} \leq \hat{\rho}_{1,0}\right\}}$ and $w_{2,0}=\mathbb{1}_{\left\{z_{0} \geq \hat{\rho}_{2,0}\right\}}$. Formally, the extracted ball is always replaced in the urn together with

$$
X_{1} D_{1,1} w_{1,0}+\left(1-X_{1}\right) D_{2,1} w_{2,0}
$$

new balls of the same color; now, the urn composition becomes

$$
\left\{\begin{array}{l}
Y_{1,1}=y_{1,0}+X_{1} D_{1,1} w_{1,0} \\
Y_{2,1}=y_{2,0}+\left(1-X_{1}\right) D_{2,1} w_{2,0}
\end{array}\right.
$$

Set $Y_{1}=Y_{1,1}+Y_{2,1}$ and $Z_{1}=Y_{1}^{-1} Y_{1,1}$. Now, by iterating the above procedure we define $\hat{\rho}_{1,1}$ and $\hat{\rho}_{2,1}$ to be two random variables, with $\hat{\rho}_{1,1}, \hat{\rho}_{2,1} \in[0,1]$ and $\hat{\rho}_{1,1} \geq \hat{\rho}_{2,1}$ a.s., measurable with respect to the $\sigma$-algebra $\mathcal{F}_{1} \equiv \sigma\left(X_{1}, \tilde{\xi}_{1}\right)$, where $\tilde{\xi}_{1}=X_{1} \xi_{1,1}+\left(1-X_{1}\right) \xi_{2,1}$. At the end of time $n$, let $\left(Y_{1, n}, Y_{2, n}\right)$ denote the urn composition and $Z_{n}=\frac{Y_{1, n}}{Y_{1, n}+Y_{2, n}}$.

Now to define the model at time $(n+1)$, let $\hat{\rho}_{1, n}$ and $\hat{\rho}_{2, n}$ be two random variables with $\hat{\rho}_{1, n}, \hat{\rho}_{2, n} \in(0,1)$ and $\hat{\rho}_{1, n} \geq \hat{\rho}_{2, n}$ a.s., measurable with respect to a $\sigma$-algebra $\mathcal{F}_{n}$, where,

$$
\mathcal{F}_{n}=\sigma\left(\mathcal{F}_{n-1}, X_{n}, \tilde{\xi}_{n}\right)
$$

and $\tilde{\xi}_{n}=X_{n} \xi_{1, n}+\left(1-X_{n}\right) \xi_{2, n}$. We will refer to $\hat{\rho}_{j, n}, j=1,2$ as threshold parameters. 
At time $(n+1)$, a ball is extracted and let $X_{n+1}=1$ if the ball is red and $X_{n+1}=0$ otherwise. Equivalently, we can define $X_{n+1}=\mathbb{1}_{\left\{U_{n+1} \leq Z_{n}\right\}}$. Then, the ball is returned to the urn together with

$$
X_{n+1} D_{1, n+1} W_{1, n}+\left(1-X_{n+1}\right) D_{2, n+1} W_{2, n}
$$

balls of the same color, where $D_{1, n+1}=u\left(\xi_{1, n+1}\right), D_{2, n+1}=u\left(\xi_{2, n+1}\right), W_{1, n}=\mathbb{1}_{\left\{Z_{n} \leq \hat{\rho}_{1, n}\right\}}$, $W_{2, n}=\mathbb{1}_{\left\{Z_{n} \geq \hat{\rho}_{2, n}\right\}}$ and $Z_{n+1}=Y_{1, n+1} / Y_{n+1}$ for any $n \geq 1$, where

$$
\left\{\begin{array}{l}
Y_{1, n+1}=y_{1,0}+\sum_{i=1}^{n+1} X_{i} D_{1, i} W_{1, i-1} \\
Y_{2, n+1}=y_{2,0}+\sum_{i=1}^{n+1}\left(1-X_{i}\right) D_{2, i} W_{2, i-1}
\end{array}\right.
$$

and $Y_{n+1}=Y_{1, n+1}+Y_{2, n+1}$. If $X_{n+1}=1$ and $Z_{n}>\hat{\rho}_{1, n}$, that is, $W_{1, n}=0$, or if $X_{n+1}=0$ and $Z_{n}<\hat{\rho}_{2, n}$, i.e. $W_{2, n}=0$, the urn composition does not change at time $n+1$. Note that condition $\hat{\rho}_{1, n} \geq \hat{\rho}_{2, n}$ a.s., which implies $W_{1, n}+W_{2, n} \geq 1$, ensures that the urn composition can change with positive probability for any $n \geq 1$, since the replacement matrix is never a zero matrix. Since, conditionally on the $\sigma$-algebra $\mathcal{F}_{n}, X_{n+1}$ is assumed to be independent of $\xi_{1}$ and $\xi_{2}$, it follows that $X_{n+1}$ is Bernoulli distributed with parameter $Z_{n}$.

Remark 2.1. Setting $\hat{\rho}_{1, n}=1$ and $\hat{\rho}_{2, n}=0$ for any $n \geq 0$, which implies $W_{1, n}=W_{2, n}=1$, equation (2.1) expresses the dynamics of an RRU, that is,

$$
\left\{\begin{array}{l}
Y_{1, n+1}=y_{1,0}+\sum_{i=1}^{n+1} X_{i} D_{1, i} \\
Y_{2, n+1}=y_{2,0}+\sum_{i=1}^{n+1}\left(1-X_{i}\right) D_{2, i} .
\end{array}\right.
$$

Setting $\hat{\rho}_{1, n}=\rho_{1}$ and $\hat{\rho}_{2, n}=\rho_{2}$ for any $n \geq 0$, which implies $W_{1, n}=\mathbb{1}_{\left\{Z_{n} \leq \rho_{1}\right\}}$ and $W_{2, n}=$ $\mathbb{1}_{\left\{Z_{n} \geq \rho_{2}\right\}}$, equation (2.1) expresses the dynamics of a MRRU, that is,

$$
\left\{\begin{array}{l}
Y_{1, n+1}=y_{1,0}+\sum_{i=1}^{n+1} X_{i} D_{1, i} \mathbb{1}_{\left\{Z_{n} \leq \rho_{1}\right\}}, \\
Y_{2, n+1}=y_{2,0}+\sum_{i=1}^{n+1}\left(1-X_{i}\right) D_{2, i} \mathbb{1}_{\left\{Z_{n} \geq \rho_{2}\right\}} .
\end{array}\right.
$$

Notice that in the MRRU $\rho_{1}$ and $\rho_{2}$ are known, while in the ARRU they are unknown and typically estimated using the data.

Before we state our results, we recall that $m_{1}=\boldsymbol{E}\left[D_{1,1}\right]$ and $m_{2}=\boldsymbol{E}\left[D_{2,1}\right]$. 


\subsection{Weak consistency of the urn proportions}

One of the main results of this paper is concerned with the consistency of the urn proportion $Z_{n}$ when the random thresholds $\hat{\rho}_{1, n}$ and $\hat{\rho}_{2, n}$ converge in probability to some constants in $\rho_{1}, \rho_{2} \in$ $(0,1)$. To obtain this result, we need to assume that the sequences of the thresholds are bounded away from 0 and 1 with exponentially high probability, which is expressed in the following condition: there exist two constants $0<\rho_{\min } \leq \rho_{\max }<1$ and $0<c_{\rho}<\infty$ such that

$$
\boldsymbol{P}\left(\rho_{\min } \leq \hat{\rho}_{2, n} \leq \hat{\rho}_{1, n} \leq \rho_{\max }\right) \geq 1-\exp \left(-c_{\rho} n\right)
$$

for large $n$. The result is described below.

Theorem 2.2. Assume (2.2) and there exist two constants $\rho_{1}, \rho_{2} \in(0,1)$, with $\rho_{1} \geq \rho_{2}$, such that

$$
\hat{\rho}_{1, n} \stackrel{p}{\rightarrow} \rho_{1}, \quad \hat{\rho}_{2, n} \stackrel{p}{\rightarrow} \rho_{2} .
$$

Then, when $m_{1} \neq m_{2}$,

$$
Z_{n} \stackrel{p}{\rightarrow} \begin{cases}\rho_{1} & \text { if } m_{1}>m_{2}, \\ \rho_{2} & \text { if } m_{1}<m_{2} .\end{cases}
$$

We present the proof of Theorem 2.2 in Section 4.

Remark 2.3. The strong consistency of the urn proportion presented in Ghiglietti, Vidyashankar and Rosenberger [16], Theorem 2.1 , that is, $\hat{\rho}_{1, n} \stackrel{\text { a.s. }}{\rightarrow} \rho_{1}$ implies $Z_{n} \stackrel{\text { a.s. }}{\rightarrow} \rho_{1}$, may suggest to prove Theorem 2.2 by applying subsequence arguments. Specifically, $Z_{n} \stackrel{p}{\rightarrow} \rho_{1}$ in (2.4) implies that for any subsequence $\left\{n_{k} ; k \geq 1\right\}$ there exists a further subsequence $\left\{n_{k_{j}} ; j \geq 1\right\}$ such that $Z_{n_{k_{j}}} \stackrel{\text { a.s. }}{\rightarrow} \rho_{1}$. Moreover, assumption $\hat{\rho}_{1, n} \stackrel{p}{\rightarrow} \rho_{1}$ in (2.3) guarantees the existence of $\left\{n_{k_{j}} ; j \geq 1\right\}$ such that $\hat{\rho}_{1, n_{k_{j}}} \stackrel{\text { a.s. }}{\rightarrow} \rho_{1}$. Nevertheless, the strong consistency result in Ghiglietti, Vidyashankar and Rosenberger [16], Theorem 2.1, does not prove that $Z_{n_{k_{j}}} \stackrel{\text { a.s. }}{\rightarrow} \rho_{1}$ with the only assumption that $\hat{\rho}_{1, n_{k_{j}}} \stackrel{\text { a.s. }}{\rightarrow} \rho_{1}$, because this condition does not provide any information on the behavior of $\hat{\rho}_{1, i}$ at times $i \notin\left\{n_{k_{j}} ; j \geq 1\right\}$. Hence, the convergence of $\hat{\rho}_{1, n_{k_{j}}}$ would imply the convergence of $Z_{n_{k_{j}}}$ only if the urn composition was updated exclusively at times $\left\{n_{k_{j}} ; j \geq 1\right\}$.

\subsection{Strong consistency of the urn proportions}

The following theorem states the consistency of the urn proportion $Z_{n}$ for all finite values of $m_{1}$ and $m_{2}$, when the random thresholds $\hat{\rho}_{1, n}$ and $\hat{\rho}_{2, n}$ converge with probability one.

Theorem 2.4. Assume there exist two constants $\rho_{1}, \rho_{2} \in[0,1]$, with $\rho_{1} \geq \rho_{2}$, such that

$$
\hat{\rho}_{1, n} \stackrel{\text { a.s. }}{\rightarrow} \rho_{1}, \quad \hat{\rho}_{2, n} \stackrel{\text { a.s. }}{\rightarrow} \rho_{2} .
$$


Then,

$$
Z_{n} \stackrel{\text { a.s. }}{\rightarrow} \begin{cases}\rho_{1} & \text { if } m_{1}>m_{2}, \\ Z_{\infty} & \text { if } m_{1}=m_{2}, \\ \rho_{2} & \text { if } m_{1}<m_{2},\end{cases}
$$

where $Z_{\infty}$ is a random variable such that $\boldsymbol{P}\left(Z_{\infty} \in\left[\rho_{2}, \rho_{1}\right]\right)=1$.

Remark 2.5. As an immediate corollary of the above theorem, it can be seen that the proportion of sampled ball colors, $n^{-1} N_{1, n}$, converges to the same limit as in (2.6).

We present the proof of Theorem 2.4 in Section 5. When the limit of the urn proportion is different from 1 or 0 , the following convergence result holds.

Lemma 2.6. Assume (2.5) with $\rho_{1}>\rho_{2}$. Then, on the set $\left\{\lim _{n \rightarrow \infty} Z_{n} \neq\{0,1\}\right\}$,

$$
\frac{Y_{n}}{n} \stackrel{\text { a.s. }}{\rightarrow} \min \left\{m_{1}, m_{2}\right\} .
$$

The above lemma can be applied for the RRU model only when $m_{1}=m_{2}$. For the case $m_{1} \neq$ $m_{2}$ in an RRU model, May and Flournoy [21] showed that $\frac{Y_{n}}{n} \stackrel{\text { a.s. }}{\rightarrow} \max \left\{m_{1} ; m_{2}\right\}$. In the case $m_{1}=m_{2}$, we are able to establish that the limiting proportion $Z_{\infty}$ has no point mass of positive probability within the open interval $\left(\rho_{2}, \rho_{1}\right)$. This is stated in the following lemma.

Lemma 2.7. Assume (2.5) with $\rho_{1}>\rho_{2}$ and $m_{1}=m_{2}$. Then, for any $x \in\left(\rho_{2}, \rho_{1}\right)$, we have $\boldsymbol{P}\left(Z_{\infty}=x\right)=0$.

Point masses of positive probability are possible at values $\rho_{1}$ and $\rho_{2}$.

\subsection{Asymptotic distribution of the sampled ball colors}

The second order asymptotic results concerning the proportion of sampled ball colors involve the concept of stable convergence (see Hall and Heyde [17]). Formally, let $\left\{\mathcal{X}_{n} ; n \geq 1\right\}$ be a random sequence on a probability space $(\Omega, \mathcal{F}, \boldsymbol{P})$; we say that $\mathcal{X}_{n} \stackrel{d}{\rightarrow} \mathcal{X}$ (stably) if, for every point $x$ of continuity for the cumulative distribution function of $\mathcal{X}$ and for every event $E \in \mathcal{F}$,

$$
\lim _{n \rightarrow \infty} \boldsymbol{P}\left(\mathcal{X}_{n} \leq x, E\right)=\boldsymbol{P}(\mathcal{X} \leq x, E)
$$

We now present the asymptotic distribution for the proportion of sampled ball colors in an RRU model. Let us denote by $N_{1 n}:=\sum_{i=1}^{n} X_{i}$ and $N_{2 n}:=\sum_{i=1}^{n}\left(1-X_{i}\right)=n-N_{1 n}$ the number of red and white balls, respectively, sampled form the urn up to time $n$. Moreover, let $\sigma_{1}^{2}:=$ $\operatorname{Var}\left[D_{1,1}\right]$ and $\sigma_{2}^{2}:=\operatorname{Var}\left[D_{2,1}\right]$. The result is stated in the following theorem. 
Theorem 2.8. Consider an RRU model and assume $m_{1}=m_{2}=m$. Then,

$$
\sqrt{n}\left(\frac{N_{1 n}}{n}-Z_{\infty}\right) \stackrel{d}{\rightarrow} \mathcal{N}(0, \Sigma) \quad(\text { stably }),
$$

where

$$
\Sigma:=\left(1+\frac{2 \bar{\Sigma}}{m^{2}}\right) Z_{\infty}\left(1-Z_{\infty}\right), \quad \bar{\Sigma}:=\left(1-Z_{\infty}\right) \sigma_{1}^{2}+Z_{\infty} \sigma_{2}^{2}
$$

Remark 2.9. In the special case of binary reinforcements with the same mean, that is, $D_{1, n} \sim$ $B e(p)$ and $D_{2, n} \sim B e(p)$ with $p \in(0,1]$, Theorem 2.8 expresses a central limit theorem with stable convergence for the RRU model studied in Durham, Flournoy and Li [12], in which $\Sigma$ in (2.7) reduces to $\left(1+2\left(\frac{1-p}{p}\right)\right) Z_{\infty}\left(1-Z_{\infty}\right)$. Then, combining Theorem 2.8 with the exact distribution of $Z_{\infty}$ established in Durham, Flournoy and Li [12], it is possible to obtain an analytic expression of the asymptotic distribution of $\sqrt{n}\left(\frac{N_{1 n}}{n}-Z_{\infty}\right)$ in this special case. A similar calculation also holds for more general binary schemes considered in Aletti, May and Secchi [4], Section 6.2.

It is known that when $D_{1, n}=D_{2, n}=1$ for any $n \geq 1$, the random variable $Z_{\infty}$ is Betadistributed with parameters $\left(y_{1,0}, y_{2,0}\right)$ (see, e.g., Athreya and Karlin [5]). Furthermore, in this case Theorem 2.8 provides a CLT with stable convergence for the standard Pòlya's urn, in which $\Sigma=Z_{\infty}\left(1-Z_{\infty}\right)$. Now, combining Theorem 2.8 with the Beta-distribution, it is possible to obtain an analytic expression of the asymptotic distribution of $\sqrt{n}\left(\frac{N_{1 n}}{n}-Z_{\infty}\right)$ for the Pòlya urn case.

We now present the asymptotic distribution for the proportion of sampled ball colors in an ARRU model. This result can be derived using Theorem 2.8 on the set of trajectories that do not cross the thresholds $\hat{\rho}_{1, n}$ and $\hat{\rho}_{2, n}$ infinitely often, and hence $\left\{Z_{\infty} \neq\left\{\rho_{2}, \rho_{1}\right\}\right\}$. To this end, we introduce a sequence of random sets $\left\{A_{n} ; n \geq 1\right\}$ such that $A_{n} \in \mathcal{F}_{n}$ and $A_{n} \subset A_{n+1}$ for any $n \geq 1$, and $\bigcup_{n \geq 1} A_{n}=\left(\rho_{2}, \rho_{1}\right)$. In particular, we fix $0<\alpha<1 / 2$ and we define $A_{n}$ as follows:

$$
A_{n}:=\left(\rho_{2}+C Y_{n}^{-\alpha}, \rho_{1}-C Y_{n}^{-\alpha}\right),
$$

where $0<C<\infty$ is a positive constant. The choice of $\left\{A_{n} ; n \geq 1\right\}$ in (2.8) allows us to apply the estimates of Lemma 3.4 in the proof of the limit distribution, in order to obtain the equivalence: $\left\{Z_{n} \in A_{n}, e v.\right\}=\left\{Z_{\infty} \in\left(\rho_{2}, \rho_{1}\right)\right\}$ a.s., where ev. stands for eventually, which means for all but a finite number of terms. The limit distribution for the ARRU model is expressed in the following result.

Theorem 2.10. Assume (2.5) with $\rho_{1}>\rho_{2}$ and $m_{1}=m_{2}=m$. Then,

$$
\underline{\lim }_{n}\left\{Z_{n} \in A_{n}\right\}=\varlimsup_{n}\left\{Z_{n} \in A_{n}\right\}=\left\{Z_{\infty} \in\left(\rho_{2}, \rho_{1}\right)\right\},
$$

and, on the sequence of sets $\left(\left\{Z_{n} \in A_{n}\right\}, n \geq 1\right)$, we have

$$
\sqrt{n}\left(\frac{N_{1 n}}{n}-Z_{\infty}\right) \stackrel{d}{\rightarrow} \mathcal{N}(0, \Sigma) \quad(\text { stably }),
$$


where, as in (2.7),

$$
\Sigma:=\left(1+\frac{2 \bar{\Sigma}}{m^{2}}\right) Z_{\infty}\left(1-Z_{\infty}\right), \quad \bar{\Sigma}:=\left(1-Z_{\infty}\right) \sigma_{1}^{2}+Z_{\infty} \sigma_{2}^{2} .
$$

It is worth noticing that the limiting distribution obtained in Theorem 2.8 and Theorem 2.10 is not Gaussian but a mixture distribution.

As a corollary of the methods of proof of Theorem 2.8 and Theorem 2.10 one can obtain the asymptotic distribution of $\sqrt{n}\left(Z_{n}-Z_{\infty}\right)$. We state this result without proof.

Theorem 2.11. Assume (2.5) with $\rho_{1}>\rho_{2}$ and $m_{1}=m_{2}=m$. Then, conditionally on $\mathcal{F}_{n}$, on the sequence of sets $\left(\left\{Z_{n} \in A_{n}\right\}, n \geq 1\right)$, we have

$$
\sqrt{n}\left(Z_{n}-Z_{\infty}\right) \stackrel{d}{\rightarrow} \mathcal{N}\left(0, \Sigma_{Z}\right) \quad(\text { stably })
$$

where

$$
\Sigma_{Z}:=\left(1+\frac{\bar{\Sigma}}{m^{2}}\right) Z_{\infty}\left(1-Z_{\infty}\right), \quad \bar{\Sigma}:=\left(1-Z_{\infty}\right) \sigma_{1}^{2}+Z_{\infty} \sigma_{2}^{2}
$$

\section{Preliminary results}

In this section, we present some preliminary estimates that are required to understand the dynamics of the ARRU model and to prove the main results of the paper. Most of the proofs of the results gathered by the literature are omitted, since the original proofs hold for all values of $m_{1}$ and $m_{2}$.

We start by presenting some basic properties of the ARRU dynamics. Specifically, we provide a useful expression of the excepted increments $\left(Z_{n+1}-Z_{n}\right)$ conditioned on $\mathcal{F}_{n}$, which is required to prove the consistency result and in particular in the proofs of Theorem 4.2 in Section 4 and of Theorem 2.8 in Section 6. Moreover, we show that the number of balls of both the colors sampled from the urn, namely $N_{1, n}$ and $N_{2, n}$, and the total number of balls in the urn $Y_{n}$, increase to infinity almost surely. To do that, we establish a lower bound for the increments of the process $Y_{n}$. These results are established in Ghiglietti, Vidyashankar and Rosenberger [16], Lemmas 4.1 and 4.2 , and we state the proof below for completeness.

Lemma 3.1. We have the following results:

(a) For any $n \geq 0$,

$$
\boldsymbol{E}\left[Z_{n+1}-Z_{n} \mid \mathcal{F}_{n}\right]=Z_{n}\left(1-Z_{n}\right) B_{n}
$$

where

$$
B_{n}:=\boldsymbol{E}\left[\frac{D_{1, n+1} W_{1, n}}{Y_{n}+D_{1, n+1} W_{1, n}}-\frac{D_{2, n+1} W_{2, n}}{Y_{n}+D_{2, n+1} W_{2, n}} \mid \mathcal{F}_{n}\right]
$$


(b) for any $n \geq 0$, we have that

$$
\boldsymbol{E}\left[Y_{n+1}-Y_{n} \mid \mathcal{F}_{n}\right] \geq \min \left\{m_{1}, m_{2}\right\} \cdot\left(\frac{\min \left\{y_{1,0} ; y_{2,0}\right\}}{y_{0}+b n}\right) ;
$$

(c)

$$
Y_{n} \stackrel{\text { a.s. }}{\rightarrow} \infty
$$

(d)

$$
\min \left\{N_{1, n} ; N_{2, n}\right\} \stackrel{\text { a.s. }}{\rightarrow} \infty
$$

Proof. The proof of result (a) is based on a modification of the proof in Muliere, Paganoni and Secchi [22], Theorem 2. First, note that, by definition

$$
Z_{n+1}=X_{n+1} \frac{Y_{1, n}+D_{1, n+1} W_{1, n}}{Y_{n}+D_{1, n+1} W_{1, n}}+\left(1-X_{n+1}\right) \frac{Y_{1, n}}{Y_{n}+D_{2, n+1} W_{2, n}}
$$

and since $X_{n+1}$ is conditionally on $\mathcal{F}_{n}$ independent of $D_{1, n+1}$ and $D_{2, n+1}$, we can get that

$$
\begin{aligned}
\boldsymbol{E}\left[Z_{n+1} \mid \mathcal{F}_{n}\right] & =Z_{n} \boldsymbol{E}\left[\frac{Y_{1, n}+D_{1, n+1} W_{1, n}}{Y_{n}+D_{1, n+1} W_{1, n}} \mid \mathcal{F}_{n}\right]+\left(1-Z_{n}\right) \boldsymbol{E}\left[\frac{Y_{1, n}}{Y_{n}+D_{2, n+1} W_{2, n}} \mid \mathcal{F}_{n}\right] \\
& =Z_{n} \boldsymbol{E}\left[\frac{Y_{1, n}+D_{1, n+1} W_{1, n}}{Y_{n}+D_{1, n+1} W_{1, n}}+\frac{Y_{2, n}}{Y_{n}+D_{2, n+1} W_{2, n}} \mid \mathcal{F}_{n}\right] .
\end{aligned}
$$

Analogously, we have that

$$
\boldsymbol{E}\left[1-Z_{n+1} \mid \mathcal{F}_{n}\right]=\left(1-Z_{n}\right) \boldsymbol{E}\left[\frac{Y_{2, n}+D_{2, n+1} W_{2, n}}{Y_{n}+D_{2, n+1} W_{2, n}}+\frac{Y_{1, n}}{Y_{n}+D_{1, n+1} W_{1, n}} \mid \mathcal{F}_{n}\right] .
$$

Therefore,

$$
\begin{aligned}
\boldsymbol{E}\left[Z_{n+1}-Z_{n} \mid \mathcal{F}_{n}\right]= & \boldsymbol{E}\left[\left(1-Z_{n}\right) Z_{n+1}-Z_{n}\left(1-Z_{n+1}\right) \mid \mathcal{F}_{n}\right] \\
= & Z_{n}\left(1-Z_{n}\right) \boldsymbol{E}\left[\frac{Y_{1, n}+D_{1, n+1} W_{1, n}}{Y_{n}+D_{1, n+1} W_{1, n}}+\frac{Y_{2, n}}{Y_{n}+D_{2, n+1} W_{2, n}}\right. \\
& \left.-\frac{Y_{2, n}+D_{2, n+1} W_{2, n}}{Y_{n}+D_{2, n+1} W_{2, n}}-\frac{Y_{1, n}}{Y_{n}+D_{1, n+1} W_{1, n}} \mid \mathcal{F}_{n}\right] \\
= & Z_{n}\left(1-Z_{n}\right) \boldsymbol{E}\left[\frac{D_{1, n+1} W_{1, n}}{Y_{n}+D_{1, n+1} W_{1, n}}-\frac{D_{2, n+1} W_{2, n}}{Y_{n}+D_{2, n+1} W_{2, n}} \mid \mathcal{F}_{n}\right] .
\end{aligned}
$$

This concludes the proof of result (a).

Concerning the proof of result (b), first note that

$$
Y_{n+1}-Y_{n}=X_{n+1} D_{1, n+1} W_{1, n}+\left(1-X_{n+1}\right) D_{2, n+1} W_{2, n} .
$$


Since $X_{n+1}$ and $D_{1, n+1}$ are conditionally independent given $\mathcal{F}_{n}$, and $W_{1, n}$ is $\mathcal{F}_{n}$-measurable, we have that

$$
\begin{aligned}
\boldsymbol{E}\left[Y_{n+1}-Y_{n} \mid \mathcal{F}_{n}\right] & =\left(m_{1} Z_{n} W_{1, n}+m_{2}\left(1-Z_{n}\right) W_{2, n}\right) \\
& \geq \min \left\{m_{1}, m_{2}\right\} \cdot\left(Z_{n} W_{1, n}+\left(1-Z_{n}\right) W_{2, n}\right) .
\end{aligned}
$$

We recall that the variables $W_{1, n}$ and $W_{2, n}$ can only take the values 0 and 1 , and by construction we have that $\left(W_{1, n}+W_{2, n}\right) \geq 1$ for any $n \geq 0$; then, we can give a further lower bound

$$
\boldsymbol{E}\left[Y_{n+1}-Y_{n} \mid \mathcal{F}_{n}\right] \geq \min \left\{m_{1}, m_{2}\right\} \cdot\left(\min \left\{Z_{n} ; 1-Z_{n}\right\}\right) .
$$

Finally, the result follows by noting that

$$
\min \left\{Z_{n} ; 1-Z_{n}\right\}=\frac{\min \left\{Y_{1, n} ; Y_{2, n}\right\}}{Y_{n}} \geq \frac{\min \left\{y_{1,0} ; y_{2,0}\right\}}{y_{0}+b n} .
$$

This concludes the proof of result (b).

We now focus on the proof of result (c). First, notice that $Y_{n}=\sum_{i=0}^{n-1}\left(Y_{i+1}-Y_{i}\right)+y_{0}$. Then, by Chen [8], Theorem 1, it is sufficient to show that

$$
\boldsymbol{P}\left(\sum_{i=0}^{\infty} \boldsymbol{E}\left[Y_{i+1}-Y_{i} \mid \mathcal{F}_{i}\right]=\infty\right)=1
$$

To this end, we will now use the lower bound given by result (b), so obtaining

$$
\sum_{i=0}^{n} \boldsymbol{E}\left[Y_{i+1}-Y_{i} \mid \mathcal{F}_{i}\right] \geq \min \left\{m_{1}, m_{2}\right\}\left(\sum_{i=0}^{n} \frac{\min \left\{y_{1,0} ; y_{2,0}\right\}}{y_{0}+b i}\right) \rightarrow \infty
$$

Hence, we have that $Y_{n} \stackrel{\text { a.s. }}{\rightarrow} \infty$.

Finally, we present the proof of result (d). We will show that $N_{1, n} \stackrel{\text { a.s. }}{\rightarrow} \infty$, since the proof for $N_{2, n}$ is analogous. Since $N_{1, n}=\sum_{i=1}^{n} X_{i}$, by Chen [8], Theorem 1, it is sufficient to show that

$$
\boldsymbol{P}\left(\sum_{i=1}^{\infty} \boldsymbol{E}\left[X_{i} \mid \mathcal{F}_{i-1}\right]=\infty\right)=1
$$

Now,

$$
\sum_{i=1}^{n} \boldsymbol{E}\left[X_{i} \mid \mathcal{F}_{i-1}\right]=\sum_{i=1}^{n} Z_{i-1} \geq \sum_{i=1}^{n} \frac{y_{1,0}}{y_{0}+(i-1) b} \rightarrow \infty .
$$

Hence, we have that $N_{1, n} \stackrel{\text { a.s. }}{\rightarrow} \infty$.

The following result is needed in the proof of Theorem 2.4 and it is taken from Aletti, Ghiglietti and Paganoni [2], Theorem 2.1. This result provides multiple equivalent ways to establish the 
almost sure convergence of a general real valued process in $[0,1]$, that we will apply to the process $\left\{Z_{n} ; n \geq 0\right\}$ of the urn proportion in an ARRU model. For this result, we let $d$ (down) and $u$ (up) be two real numbers such that $0<d<u<1$, and we consider two sequences of times $t_{j}(d, u)$ and $\tau_{j}(d, u)$ defined as follows: for each $j \geq 0, t_{j}(d, u)$ represents the time of the first up-cross of $u$ after $\tau_{j-1}(d, u)$, and $\tau_{j}(d, u)$ represents the time of the first down-cross of $d$ after $t_{j}$. Note that $t_{j}(d, u)$ and $\tau_{j}(d, u)$ are stopping times, since the events $\left\{t_{j}(d, u)=k\right\}$ and $\left\{\tau_{j}(d, u)=k\right\}$ depend on $\left\{Z_{n} ; n \leq k\right\}$, which are measurable with respect to $\mathcal{G}_{k}=\sigma\left(Z_{n}\right.$ : $n \leq k)$.

Lemma 3.2 (Aletti, Ghiglietti and Paganoni [2], Theorem 2.1). Let $\tau_{-1}(d, u)=-1$ and define for every $j \geq 0$ two stopping times

$$
\begin{aligned}
t_{j}(d, u) & = \begin{cases}\inf \left\{n>\tau_{j-1}(d, u): Z_{n}>u\right\} & \text { if }\left\{n>\tau_{j}(d, u): Z_{n}>u\right\} \neq \varnothing \\
+\infty & \text { otherwise, }\end{cases} \\
\tau_{j}(d, u) & = \begin{cases}\inf \left\{n>t_{j}(d, u): Z_{n}<d\right\} & \text { if }\left\{n>t_{j-1}(d, u): Z_{n}<d\right\} \neq \varnothing \\
+\infty & \text { otherwise. }\end{cases}
\end{aligned}
$$

Then, the following three events are a.s. equivalent

(a) $Z_{n}$ converges a.s.;

(b) for any $0<d<u<1$,

$$
\lim _{j \rightarrow \infty} \boldsymbol{P}\left(t_{j}(d, u)<\infty\right)=0
$$

(c) for any $0<d<u<1$,

$$
\sum_{j \geq 1} \boldsymbol{P}\left(t_{j+1}(d, u)=\infty \mid t_{j}(d, u)<\infty\right)=\infty
$$

using the convention that $\boldsymbol{P}\left(t_{j+1}(d, u)=\infty \mid t_{j}(d, u)<\infty\right)=1$ when $\boldsymbol{P}\left(t_{j}(d, u)=\infty\right)=1$.

We now present a lemma that provides lower bounds for the total number of balls in the urn at the times of up-crossings, $Y_{t_{j}}$. The lemma gets used in the proof of Theorem 2.4, where conditioning on a fixed number of up-crossing ensures to have at least a number of balls $Y_{n}$ determined by the lower bounds of this lemma. This result has been taken by Aletti, Ghiglietti and Paganoni [2], Lemma 2.1, and the proof is omitted since the adaptive thresholds and the values of $m_{1}$ and $m_{2}$ do not play any role during up-crossings. Hence, the proof reported in Aletti, Ghiglietti and Paganoni [2], Lemma 2.1, carries over to our model, with $D_{n}$ replaced by $Y_{n}$.

Lemma 3.3 (Aletti, Ghiglietti and Paganoni [2], Lemma 2.1). For any $0<d<u<1$, we have that

$$
Y_{t_{j}(d, u)} \geq\left(\frac{u(1-d)}{d(1-u)}\right) Y_{t_{j-1}(d, u)} \geq \cdots \geq\left(\frac{u(1-d)}{d(1-u)}\right)^{j} Y_{t_{0}(d, u)}
$$


The following lemma provides a uniform bound for the generalized Pólya urn with same reinforcement means, which is needed in the proof of Theorem 2.4.

Lemma 3.4 (Aletti, Ghiglietti and Paganoni [2], Lemma 3.2). Consider an $R R U$ with $m_{1}=$ $m_{2}$. If $Y_{0} \geq 2 b$, then

$$
\boldsymbol{P}\left(\sup _{n \geq 1}\left|Z_{n}-Z_{0}\right| \geq h\right) \leq \frac{b}{Y_{0}}\left(\frac{4}{h^{2}}+\frac{2}{h}\right)
$$

for every $h>0$.

Finally, we present an auxiliary result that provides an upper bound on the increments of the urn process $Z_{n}$, by imposing a condition on the total number of balls in the urn $Y_{n}$.

Lemma 3.5 (Ghiglietti, Vidyashankar and Rosenberger [16], Lemma 3.1). For any $\epsilon \in$ $(0,1)$, we have that

$$
\left\{Y_{n}>b\left(\frac{1-\epsilon}{\epsilon}\right)\right\} \subseteq\left\{\left|Z_{n+1}-Z_{n}\right|<\epsilon\right\} .
$$

\section{Proof of weak consistency and related results}

In this section, we prove the weak consistency for the urn proportion of the ARRU model, which is established in Theorem 2.2. This proof requires some probabilistic results concerning the ARRU model, which have been gathered in different subsections. The proof of the weak consistency based on these results is then provided in Section 4.4.

Let us start by describing the general structure of the proof. The weak consistency is proved by showing that the process $\left\{\Delta_{n} ; n \geq 1\right\}$, defined as

$$
\Delta_{n}:=\left|\rho_{1}-Z_{n}\right|, \quad \forall n \geq 0,
$$

converges to zero in probability. To prove this, we want to exploit the fact that, unless $\Delta_{n}$ is arbitrarily close to zero, the conditional expected increments of $\Delta_{n}$ are negative. This result is obtained in Section 4.2 by studying the conditional expected increments of $Z_{n}$. Hence, to show that $\Delta_{n}$ is asymptotically close to zero, we need to investigate the expected increments of the process $\left\{\Delta_{n} ; n \geq 1\right\}$. Since the increments of $\Delta_{n}$ are at the same order of $Y_{n}^{-1}$, we first determine how fast the total number of balls in the urn, $Y_{n}$, increases to infinity. This is addressed in Theorem 4.1, where we show that the total number of balls in the ARRU model increases linearly with the number of extractions from the urn. For this reason, the increments of $\Delta_{n}$ are of the order of $n^{-1}$; hence, we consider differences of $\Delta_{n}$ evaluated at linearly increasing times, that is, $G(n, c):=\left(\Delta_{n+n c}-\Delta_{n}\right)$, such that the $L_{1}$ bounds obtained for such differences do not vanish as $n$ goes to infinity. More specifically, we provide a negative upper bound for the expected differences $G(n, c)$, which is not negligible unless $\Delta_{n}$ is asymptotically close to zero. Formally, 
for any $\delta>0$, we show that for some $0<C<\infty$

$$
\boldsymbol{E}\left[G\left(n, s_{\delta}\right)\right] \leq-C \boldsymbol{P}(Q(\delta, n))+o(1),
$$

where $0<s_{\delta}<\infty$ is an appropriate constant and $Q(\delta, n):=\left\{\Delta_{n}>\delta\right\}$. To obtain (4.2), we prove that the expected differences $G\left(n, s_{\delta}\right)$ are: (i) negative for moderate values of $\Delta_{n}$ (see Theorem 4.5) and (ii) negligible for small values of $\Delta_{n}$ (see Theorem 4.6). These results are derived using comparison arguments with specific auxiliary urn models. Finally, in Section 4.4 we use (4.2) and other preliminary results to establish the weak consistency.

\subsection{Harmonic moments of $Y_{n}$}

In this subsection, we establish that the total number of balls in the ARRU model increases linearly with the number of extractions from the urn. Moreover, this result ensures uniform bounds for the harmonic moments of the total number of balls.

Before presenting the main result, we introduce some notation. For any $0<c \leq C<\infty$ and for all $n \geq 0$, let $F_{n}(c, C) \in \mathcal{F}_{n}$ be the set defined as follows

$$
F_{n}(c, C):=\left\{y_{0}+c n \leq Y_{n} \leq y_{0}+C n\right\} .
$$

Here, we show that, for some $c$ and $C, \boldsymbol{P}\left(F_{n}(c, C)\right)$ converges to one with a sub-exponential rate (a sequence of constants $a_{n}$ is said to converge at a sub-exponential rate to 1 (resp. 0) if $a_{n} \geq 1-$ $\exp \left(-c n^{p}\right)\left(\right.$ resp. $\left.a_{n} \leq \exp \left(-c n^{p}\right)\right)$ for some $\left.0<p<1\right)$, which implies $\boldsymbol{P}\left(F_{n}^{c}(c, C)\right.$, i.o. $)=0$. Moreover, this result provides uniform bounds for the moments of $n / Y_{n}$. The following theorem makes this result precise.

Theorem 4.1. Under assumption (2.2), there exist two constants $0<z_{\min }<\rho_{\min }$ and $\rho_{\max }<$ $z_{\max }<1$ such that, for some $\epsilon_{z}>0$, depending on $z_{\min }$ and $z_{\max }$, we have for large $n$ that

$$
\boldsymbol{P}\left(z_{\min } \leq Z_{n} \leq z_{\max }\right) \geq 1-\exp \left(-\epsilon_{z} \sqrt{n}\right) .
$$

Moreover, there exist two constants $0<c_{1}<C_{1}<\infty$ such that, for some $\epsilon_{y}>0$, depending on $y_{0}$, we have for large $n$ that

$$
F_{n}\left(c_{1}, C_{1}\right) \equiv \boldsymbol{P}\left(y_{0}+c_{1} n \leq Y_{n} \leq y_{0}+C_{1} n\right) \geq 1-\exp \left(-\epsilon_{y} \sqrt{n}\right)
$$

As a consequence, for any $j \geq 1$

$$
\sup _{n \geq 0}\left\{\boldsymbol{E}\left[\left(\frac{n}{Y_{n}}\right)^{j}\right]\right\}<\infty .
$$

Proof. This proof has a general structure similar to the proof in Ghiglietti, Vidyashankar and Rosenberger [16], Theorem 3.1. 
Let $c_{\min }:=\min \left\{\rho_{\min } ; 1-\rho_{\max }\right\}$ and $p_{0}:=\left(\frac{y_{0}}{y_{0}+b}\right) c_{\min }<c_{\min }$. Fix an arbitrary $0<c<p_{0}$ and consider the following sets

$$
\begin{aligned}
A_{d, n} & :=\bigcup_{n / 2 \leq i \leq n}\left\{Z_{i}<c\right\}, \\
A_{c, n} & :=\bigcap_{n / 2 \leq i \leq n}\left\{c<Z_{i}<1-c\right\}, \\
A_{u, n} & :=\bigcup_{n / 2 \leq i \leq n}\left\{Z_{i}>1-c\right\} .
\end{aligned}
$$

Note that by choosing $0<z_{\min } \leq c$ and $1-c \leq z_{\max }<1$ we have $A_{c, n} \subset\left\{z_{\min } \leq Z_{n} \leq z_{\max }\right\}$. Then, since $A_{d, n} \cup A_{c, n} \cup A_{u, n}=\Omega$, result (4.3) can be established by proving that $\boldsymbol{P}\left(A_{d, n}\right)+$ $\boldsymbol{P}\left(A_{u, n}\right) \leq \exp \left(-\epsilon_{z} \sqrt{n}\right)$ for large $n$. We will focus on the set $A_{d, n}$, since the arguments to deal with $A_{u, n}$ are analogous. First, define $\epsilon>0$ and, for any $n \geq 1$, the following sets:

$$
\begin{aligned}
& A_{1, n}:=\left\{\sup _{i \geq \epsilon \sqrt{n}}\left\{\hat{\rho}_{1, i}\right\}>1-c_{\min }\right\}, \\
& A_{2, n}:=\left\{\inf _{i \geq \epsilon \sqrt{n}}\left\{\hat{\rho}_{2, i}\right\}<c_{\min }\right\}, \\
& A_{3, n}:=\left\{\inf _{i \geq \epsilon \sqrt{n}}\left\{\min \left\{1-\hat{\rho}_{1, i} ; \hat{\rho}_{2, i}\right\}\right\} \geq c_{\min }\right\} .
\end{aligned}
$$

Note that $A_{1, n} \cup A_{2, n} \cup A_{3, n}=\Omega$, and hence we have that

$$
\boldsymbol{P}\left(A_{d, n}\right) \leq \boldsymbol{P}\left(A_{1, n}\right)+\boldsymbol{P}\left(A_{2, n}\right)+\boldsymbol{P}\left(A_{3, n} \cap A_{d, n}\right) .
$$

First, we prove that $\boldsymbol{P}\left(A_{1, n}\right)$ and $\boldsymbol{P}\left(A_{2, n}\right)$ converge to zero with a sub-exponential rate. Consider the term $\boldsymbol{P}\left(A_{1, n}\right)$. From the definition of $A_{1, n}$, we obtain

$$
\boldsymbol{P}\left(A_{1, n}\right)=\boldsymbol{P}\left(\bigcup_{i \geq \epsilon \sqrt{n}}\left\{\hat{\rho}_{1, i}>1-c_{\min }\right\}\right) \leq \sum_{i \geq \epsilon \sqrt{n}} \boldsymbol{P}\left(\hat{\rho}_{1, i}>1-c_{\min }\right) .
$$

Since, $\left(1-c_{\min }\right) \geq \rho_{\max }$, from (2.2) we have that for large $i$

$$
\boldsymbol{P}\left(\hat{\rho}_{1, i}>1-c_{\min }\right) \leq \exp \left(-c_{\rho} i\right)
$$

with $0<c_{\rho}<\infty$. Hence, we have that

$$
\begin{aligned}
\boldsymbol{P}\left(A_{1, n}\right) & \leq \sum_{i \geq \epsilon \sqrt{n}} \boldsymbol{P}\left(\hat{\rho}_{1, i}>1-c_{\min }\right) \\
& \leq \sum_{i \geq \epsilon \sqrt{n}} \exp \left(-c_{\rho} i\right) \\
& \leq \exp \left(-\epsilon_{z} \sqrt{n}\right),
\end{aligned}
$$


for some constat $\epsilon_{z}>0$. Similar arguments can be applied to prove $\boldsymbol{P}\left(A_{2, n}\right) \rightarrow 0$ with a subexponential rate.

Finally, we need to show that $\boldsymbol{P}\left(A_{3, n} \cap A_{d, n}\right)$ converges to zero with a sub-exponential rate. First, define the set $\tilde{A}_{d, n}$ as follows:

$$
\tilde{A}_{d, n}:=\bigcap_{\epsilon \sqrt{n} \leq i \leq n / 2}\left\{Z_{i}<c\right\}
$$

We now show that, since $0<c<p_{0}=c_{\min }\left(\frac{y_{0}}{b+y_{0}}\right)$, it follows that on the set $A_{3, n}$ we have $\left\{Z_{i} \geq c\right\} \subset\left\{Z_{i+1} \geq c\right\}$ for any $i \geq \epsilon \sqrt{n}$, and hence

$$
\left(A_{3, n} \cap A_{d, n}\right) \subset\left(A_{3, n} \cap \tilde{A}_{d, n}\right),
$$

for any $n \geq 1$. First, note that on the set $A_{3, n},\left\{\hat{\rho}_{2, i} \geq c_{\min }\right\}$ for any $i \geq \epsilon \sqrt{n}$. Hence for any $0<c<p_{0}<c_{\min }$, since $\left\{Z_{i} \geq c\right\}=\left\{c \leq Z_{i}<c_{\min }\right\} \cup\left\{Z_{i} \geq c_{\min }\right\}$, we have

(1) if $\left\{c \leq Z_{i}<c_{\min }\right\}$ we have $W_{2, i}=0$, which implies $Z_{i+1} \geq Z_{i}$ and so $Z_{i+1} \geq c$;

(2) if $\left\{Z_{i} \geq c_{\min }\right\}$, the event $\left\{Z_{i+1} \leq Z_{i}\right\}$ is possible but using $X_{i+1} \geq 0, W_{2, i} \leq 1, D_{2, i+1} \leq b$ and $Y_{i} \geq y_{0}$ we obtain

$$
\begin{aligned}
Z_{i+1} & =\frac{Z_{i} Y_{i}+X_{i+1} D_{1, i+1} W_{1, i}}{Y_{i}+X_{i+1} D_{1, i+1} W_{1, i}+\left(1-X_{i+1}\right) D_{2, i+1} W_{2, i}} \\
& \geq \frac{Z_{i} y_{0}}{y_{0}+b} \geq c_{\min } \frac{y_{0}}{y_{0}+b}=p_{0}>c .
\end{aligned}
$$

This guarantees that (4.6) holds for any $n \geq 1$.

We now show that $\boldsymbol{P}\left(A_{3, n} \cap \tilde{A}_{d, n}\right)$ converges to zero with a sub-exponential rate. To this end, first note that on the set $A_{3, n}$, we have $\hat{\rho}_{2, i} \geq c_{\min }$ for any $i=\epsilon \sqrt{n}, \ldots, n / 2$; moreover, on the set $\tilde{A}_{d, n}$, we have $Z_{i}<p_{0}$ for any $i=\epsilon \sqrt{n}, \ldots, n / 2$. These considerations imply that

$$
W_{2, i}=0 \quad \text { and } \quad W_{1, i}=1 \quad \text { for any } i=\epsilon \sqrt{n}, \ldots, n / 2 \text {, on the set } A_{3, n} \cap \tilde{A}_{d, n} .
$$

Hence, we can write

$$
Z_{n / 2}=\frac{Y_{1, \epsilon \sqrt{n}}+\sum_{i=\epsilon \sqrt{n}}^{n / 2} X_{i} D_{1, i}}{Y_{\epsilon \sqrt{n}}+\sum_{i=\epsilon \sqrt{n}}^{n / 2} X_{i} D_{1, i}} \geq \frac{a \sum_{i=\epsilon \sqrt{n}}^{n / 2} X_{i}}{\left(y_{0}+b \epsilon \sqrt{n}\right)+a \sum_{i=\epsilon \sqrt{n}}^{n / 2} X_{i}},
$$

where the inequality follows since $0 \leq Y_{1, \epsilon \sqrt{n}}<Y_{\epsilon \sqrt{n}} \leq y_{0}+b \epsilon \sqrt{n}, D_{1, i} \geq a$ a.s. for any $i \geq 1$ and the function $\frac{c+x}{C+x}$ is increasing for $x>0$ and $c<C$. Now, define for any $n \geq 1$ the set $A_{4, n}$ as follows:

$$
A_{4, n}:=\left\{\sum_{i=\epsilon \sqrt{n}}^{n / 2} X_{i}>\frac{p_{0}}{a\left(1-p_{0}\right)}\left(y_{0}+b \epsilon \sqrt{n}\right)\right\}
$$


and consider the set $A_{3, n} \cap \tilde{A}_{d, n} \cap A_{4, n}$. On the set $A_{3, n} \cap \tilde{A}_{d, n}$ we can use the definition of $A_{4, n}$ in (4.8), so obtaining

$$
\left(A_{3, n} \cap \tilde{A}_{d, n} \cap A_{4, n}\right) \subset\left(\left\{Z_{n / 2}>p_{0}\right\} \cap \tilde{A}_{d, n}\right) .
$$

However, $\left\{Z_{n / 2}>p_{0}\right\} \cap \tilde{A}_{d, n}=\varnothing$. Hence, $\boldsymbol{P}\left(A_{3, n} \cap \tilde{A}_{d, n} \cap A_{4, n}\right)=0$ and it is sufficient to show that $\boldsymbol{P}\left(A_{3, n} \cap \tilde{A}_{d, n} \cap A_{4, n}^{C}\right)$ converges to zero with a sub-exponential rate.

To this end, by (4.7), note that on the set $A_{3, n} \cap \tilde{A}_{d, n}$ we have $Z_{i+1} \geq Z_{i}$ for any $i=$ $\epsilon \sqrt{n}, \ldots, n / 2$. Hence, on the set $A_{3, n} \cap \tilde{A}_{d, n},\left\{X_{i}, i=\epsilon \sqrt{n}, \ldots, n / 2\right\}$ are conditionally Bernoulli with parameter $p_{i} \geq Z_{\epsilon \sqrt{n}}$ a.s. Now, let us denote with $\left\{\varrho_{i, n} ; i=1, \ldots, n / 2-\epsilon \sqrt{n}\right\}$ a sequence of i.i.d. Bernoulli random variable with parameter $z_{0, n}$, defined as

$$
z_{0, n}:=\frac{y_{1,0}}{y_{0}+b \epsilon \sqrt{n}} \leq Z_{\epsilon \sqrt{n}} \quad \text { a.s.; }
$$

it follows that

$$
\boldsymbol{P}\left(A_{3, n} \cap \tilde{A}_{d, n} \cap A_{4, n}^{C}\right) \leq \boldsymbol{P}\left(\sum_{i=1}^{n / 2-\epsilon \sqrt{n}} \varrho_{i, n} \leq \frac{p_{0}}{a\left(1-p_{0}\right)}\left(y_{0}+b \epsilon \sqrt{n}\right)\right) .
$$

Finally, we use the following Chernoff's upper bound for i.i.d. random variables in $[0,1]$ (see Dembo and Zeitouni [11])

$$
\boldsymbol{P}\left(S_{n} \leq c_{0} \cdot \boldsymbol{E}\left[S_{n}\right]\right) \leq \exp \left(-\frac{\left(1-c_{0}\right)^{2}}{2} \cdot \boldsymbol{E}\left[S_{n}\right]\right),
$$

with $c_{0} \in(0,1)$ and $S_{n}=\sum_{i}^{n} X_{i}$. In our case, we have that RHS of (4.9) can be written as $\boldsymbol{P}\left(S_{n} \leq c_{n} \cdot \boldsymbol{E}\left[S_{n}\right]\right)$, where $S_{n}=\sum_{i=1}^{n / 2-\epsilon \sqrt{n}} \varrho_{i, n}$,

$$
\boldsymbol{E}\left[S_{n}\right]=\left(\frac{n}{2}-\epsilon \sqrt{n}\right) \frac{y_{1,0}}{\left(y_{0}+b \epsilon \sqrt{n}\right)} \quad \text { and } \quad c_{n}=\frac{p_{0}}{a\left(1-p_{0}\right)} \frac{\left(y_{0}+b \epsilon \sqrt{n}\right)^{2}}{y_{1,0}(n / 2-\epsilon \sqrt{n})} ;
$$

since $\epsilon>0$ can be chosen arbitrary small, we can define an integer $n_{0} \geq 1$ and a constant $c_{0} \epsilon$ $(0,1)$ such that $c_{n}<c_{0}$ for any $n \geq n_{0}$, so that

$$
\boldsymbol{P}\left(S_{n} \leq c_{n} \cdot \boldsymbol{E}\left[S_{n}\right]\right) \leq \boldsymbol{P}\left(S_{n} \leq c_{0} \cdot \boldsymbol{E}\left[S_{n}\right]\right) .
$$

Hence, by using (4.10), for any $n \geq n_{0}$ we have that

$$
\boldsymbol{P}\left(A_{3, n} \cap A_{4, n}^{C}\right) \leq \exp \left(-\frac{\left(1-c_{0}\right)^{2}}{2} \cdot \boldsymbol{E}\left[S_{n}\right]\right),
$$

which converges to zero with a sub-exponential rate since

$$
\boldsymbol{E}\left[S_{n}\right]=\frac{y_{1,0}(n / 2-\epsilon \sqrt{n})}{y_{0}+b \epsilon \sqrt{n}} \sim \frac{n}{\sqrt{n}}=\sqrt{n} .
$$

This concludes the proof of (4.3). 
Now, we prove (4.4). Since the reinforcements are a.s. bounded, that is, $\left|D_{j, n}\right|<b$ for any $n \geq 1$ and $j=1,2$, we trivially have that $\boldsymbol{P}\left(Y_{n} \geq y_{0}+n b\right)=0$. Thus, we will show that $\boldsymbol{P}\left(Y_{n}-\right.$ $\left.y_{0} \leq c_{1} n\right)$ converges to zero with a sub-exponential rate. Moreover, since in the proof of (4.3) we established that for any $0<c<p_{0}$ there exists $\epsilon_{z}$ such that $\boldsymbol{P}\left(A_{c, n}\right) \geq 1-\exp \left(-\epsilon_{z} \sqrt{n}\right)$, then to prove (4.4) we can focus on the probability $\boldsymbol{P}\left(\left\{Y_{n}-y_{0} \leq c_{1} n\right\} \cap A_{c, n}\right)$.

First, consider the following relation on the increments of the total number of balls

$$
\begin{aligned}
Y_{i}-Y_{i-1} & =D_{1, i} X_{i} W_{1, i-1}+D_{2, i}\left(1-X_{i}\right) W_{2, i-1} \\
& \geq a\left[X_{i} W_{1, i-1}+\left(1-X_{i}\right) W_{2, i-1}\right]
\end{aligned}
$$

Then, note that, on the set $A_{c, n}$, the random variables

$$
X_{i} W_{1, i-1}+\left(1-X_{i}\right) W_{2, i-1}, \quad i=n / 2, \ldots, n
$$

are, conditionally on the $\sigma$-algebra $\mathcal{F}_{i-1}$, Bernoulli with parameter greater than or equal to $c$. Hence, if we introduce $\left\{B_{i} ; i \geq 1\right\}$ a sequence of i.i.d. Bernoulli random variables with parameter $c$, using that $Y_{n}$ is increasing we have

$$
\begin{aligned}
\boldsymbol{P}\left(\left\{Y_{n}-y_{0} \leq c_{1} n\right\} \cap A_{c, n}\right) & \leq \boldsymbol{P}\left(\left\{Y_{n}-Y_{n / 2} \leq c_{1} n\right\} \cap A_{c, n}\right) \\
& \leq \boldsymbol{P}\left(\left\{a \sum_{i=n / 2}^{n} B_{i} \leq c_{1} n\right\} \cap A_{c, n}\right) \\
& \leq \boldsymbol{P}\left(\sum_{i=n / 2}^{n} B_{i} \leq \frac{c_{1}}{a} n\right)
\end{aligned}
$$

Now, we want to use the Chernoff's bound for i.i.d. random variables in $[0,1]$ expressed in (4.10), with $S_{n}=\sum_{i=n / 2}^{n} B_{i}$. In our case, we have $\boldsymbol{E}\left[S_{n}\right]=n c / 2$ and so $c_{0}=2 c_{1} /(a c)$. Hence, by choosing $c_{1}$ small enough we can obtain $c_{0}<1$ which let us apply Chernoff's bound. This implies (4.4).

Finally, we get the harmonic moments as follows

$$
\begin{aligned}
\boldsymbol{E}\left[\left(\frac{n}{Y_{n}}\right)^{j}\right] & =\boldsymbol{E}\left[\left(\frac{n}{Y_{n}}\right)^{j} \mathbb{1}_{F_{n}\left(c_{1}, C_{1}\right)}\right]+\boldsymbol{E}\left[\left(\frac{n}{Y_{n}}\right)^{j} \mathbb{1}_{F_{n}^{c}\left(c_{1}, C_{1}\right)}\right] \\
& \leq \boldsymbol{E}\left[\left(\frac{n}{y_{0}+c_{1} n}\right)^{j} \mathbb{1}_{F_{n}\left(c_{1}, C_{1}\right)}\right]+\left(\frac{n}{y_{0}}\right)^{j} \boldsymbol{E}\left[\mathbb{1}_{F_{n}^{c}\left(c_{1}, C_{1}\right)}\right] \\
& \leq c_{1}^{-j}+y_{0}^{-j} n^{j} \exp \left(-\epsilon_{y} \sqrt{n}\right) .
\end{aligned}
$$

We notice here that the above proof also works for the case $a=0$ by directly working with $\sum_{i=1}^{n} X_{i} D_{1, i}$ and using general large deviation estimates in (4.9) and (4.11). 


\section{2. $L_{1}$ bound for the increments of $\Delta_{n}$}

To ease notation in the rest of paper, we will refer to $F_{n}\left(c_{1}, C_{1}\right)$ as

$$
F_{n}:=\left\{y_{0}+c_{1} n \leq Y_{n} \leq y_{0}+C_{1} n\right\},
$$

where $0<c_{1}<C_{1}<\infty$ are the constants determined in Theorem 4.1 to obtain (4.4). Also, for any $\varepsilon>0$, let $R(\varepsilon, n):=\left\{\left|\hat{\rho}_{1, n}-\rho_{1}\right|<\varepsilon\right\}$ and $Q(\varepsilon, n):=\left\{\Delta_{n}>\varepsilon\right\}$, where we recall from (4.1) that $\Delta_{n}=\left|\rho_{1}-Z_{n}\right|$. The following result provides an upper bound on the increments of $\Delta_{n}$.

Theorem 4.2. Let $m_{1}>m_{2}$ and assume (2.2) and (2.3). For any $\varepsilon>0$, there exists $0<c_{2}<\infty$ and a sequence of random variables $\left\{\psi_{n} ; n \geq 0\right\}$ with $\boldsymbol{E}\left[\left|\psi_{n}\right|\right]=o\left(n^{-1}\right)$, such that

$$
\boldsymbol{E}\left[G\left(n, n^{-1}\right) \mathbb{1}_{Q(\varepsilon, n)} \mid \mathcal{F}_{n}\right] \leq-n^{-1} \cdot c_{2} \mathbb{1}_{Q(\varepsilon, n)}+\psi_{n},
$$

where we recall $G\left(n, n^{-1}\right)=\left(\Delta_{n+1}-\Delta_{n}\right)$.

The behavior and the sign of the excepted increments of the urn proportion $G\left(n, n^{-1}\right)$ required to prove Theorem 4.2 depend on the position of $Z_{n}$ respect to $\rho_{1}$. For this reason, we study separately the cases when $Z_{n}$ is above or below $\rho_{1}$. Formally, we define

$$
Q^{-}(\varepsilon, n):=\left\{Z_{n}<\rho_{1}-\varepsilon\right\}, \quad Q^{+}(\varepsilon, n):=\left\{Z_{n}>\rho_{1}+\varepsilon\right\},
$$

so that $Q(\varepsilon, n)=Q^{+}(\varepsilon, n) \cup Q^{-}(\varepsilon, n)$. Specifically, we present Lemma 4.3 and Lemma 4.4 that provide bounds for the expected increments $G\left(n, n^{-1}\right)$ on the sets $Q^{-}(\varepsilon, n)$ and $Q^{+}(\varepsilon, n)$, respectively. The proof of Theorem 4.2 is presented after the proofs of Lemma 4.3 and Lemma 4.4.

Lemma 4.3. Let $A_{n} \in \mathcal{F}_{n}$ be such that $A_{n} \subset Q^{-}(\varepsilon, n)$. Then, we have that

$$
\boldsymbol{E}\left[\left(Z_{n+1}-Z_{n}\right) \mathbb{1}_{A_{n}}\right] \geq n^{-1} \cdot c_{2} \boldsymbol{P}\left(A_{n}\right)-o\left(n^{-1}\right) .
$$

Proof. Let $I_{n}:=\boldsymbol{E}\left[\left(Z_{n+1}-Z_{n}\right) \mathbb{1}_{A_{n}}\right]$ and, since $A_{n} \in \mathcal{F}_{n}$, we can use result (a) of Lemma 3.1 obtaining

$$
I_{n}=\boldsymbol{E}\left[\boldsymbol{E}\left[Z_{n+1}-Z_{n} \mid \mathcal{F}_{n}\right] \mathbb{1}_{A_{n}}\right]=\boldsymbol{E}\left[Z_{n}\left(1-Z_{n}\right) B_{n} \mathbb{1}_{A_{n}}\right],
$$

where we recall that $B_{n}$ is defined in (3.1) as follows

$$
B_{n}:=\boldsymbol{E}\left[\frac{D_{1, n+1} W_{1, n}}{Y_{n}+D_{1, n+1} W_{1, n}}-\frac{D_{2, n+1} W_{2, n}}{Y_{n}+D_{2, n+1} W_{2, n}} \mid \mathcal{F}_{n}\right] .
$$

Now, note the following relation

$$
\left\{Z_{n} \leq \hat{\rho}_{1, n}\right\} \supset\left(Q^{-}(\varepsilon, n) \cap R(\varepsilon, n)\right),
$$


where $R(\varepsilon, n)=\left\{\left|\hat{\rho}_{1, n}-\rho_{1}\right|<\varepsilon\right\}$. Since $A_{n} \subset Q^{-}(\varepsilon, n)$, on the set $A_{n}$ the previous relation becomes $\left\{Z_{n} \leq \hat{\rho}_{1, n}\right\} \supset R(\varepsilon, n)$, which implies $W_{1, n} \geq \mathbb{1}_{R(\varepsilon, n)}$. Combining this argument with $W_{2, n} \leq 1$, we obtain on the set $A_{n}$ the following inequality

$$
B_{n} \geq \boldsymbol{E}\left[\left(\frac{D_{1, n+1} \mathbb{1}_{R(\varepsilon, n)}}{Y_{n}+D_{1, n+1} \mathbb{1}_{R(\varepsilon, n)}}-\frac{D_{2, n+1}}{Y_{n}+D_{2, n+1}}\right) \mid \mathcal{F}_{n}\right] .
$$

Then, by using $D_{2, n+1} \geq 0$ and $D_{1, n+1} \mathbb{1}_{R(\varepsilon, n)} \leq b$ a.s., we obtain that, on the set $A_{n}$,

$$
B_{n} \geq \boldsymbol{E}\left[\left(\frac{D_{1, n+1} \mathbb{1}_{R(\varepsilon, n)}}{Y_{n}+b}-\frac{D_{2, n+1}}{Y_{n}}\right) \mid \mathcal{F}_{n}\right]=E_{1 n}-E_{2 n},
$$

where

$$
E_{1 n}:=\frac{m_{1} \mathbb{1}_{R(\varepsilon, n)}-m_{2}}{Y_{n}+b} \quad \text { and } \quad E_{2 n}:=\frac{m_{2} b}{Y_{n}\left(Y_{n}+b\right)}
$$

First, note that

$$
\boldsymbol{E}\left[Z_{n}\left(1-Z_{n}\right) E_{2 n} \mathbb{1}_{A_{n}}\right] \leq \boldsymbol{E}\left[E_{2 n}\right] \leq m_{2} b \sup _{k \geq 1} \boldsymbol{E}\left[\left(\frac{k}{Y_{k}}\right)^{2}\right] n^{-2} .
$$

Now, using (4.5) it follows that

$$
\boldsymbol{E}\left[Z_{n}\left(1-Z_{n}\right) E_{2 n} \mathbb{1}_{A_{n}}\right]=O\left(n^{-2}\right) .
$$

Thus, from (4.16) we have

$$
I_{n} \geq \boldsymbol{E}\left[Z_{n}\left(1-Z_{n}\right) E_{1 n} \mathbb{1}_{A_{n}}\right]-o\left(n^{-1}\right) .
$$

Now, consider the set $F_{n}$ defined in (4.12) as

$$
F_{n}=\left\{c_{1} n \leq Y_{n}-y_{0} \leq C_{1} n\right\},
$$

where we recall that, by (4.4) in Theorem 4.1, $\boldsymbol{P}\left(F_{n}^{c}\right) \leq \exp \left(-\epsilon_{y} \sqrt{n}\right)$. Moreover, let $\mathbb{1}_{A_{n}}=$ $J_{1 n}+J_{2 n}$, where $J_{1 n}:=\mathbb{1}_{A_{n} \cap F_{n}}$ and $J_{2 n}:=\mathbb{1}_{A_{n} \cap F_{n}^{c}}$. Thus, concerning $J_{2 n}$ we have that

$$
\left|\boldsymbol{E}\left[Z_{n}\left(1-Z_{n}\right) E_{1 n} J_{2 n}\right]\right| \leq \max _{n \geq 0}\left\{\left|E_{1 n}\right|\right\} \boldsymbol{P}\left(F_{n}^{c}\right)=o\left(n^{-1}\right),
$$

since $\max _{n \geq 0}\left\{\left|E_{1 n}\right|\right\} \leq b / y_{0}$ a.s. Thus, returning to (4.17) we have that

$$
I_{n} \geq \boldsymbol{E}\left[Z_{n}\left(1-Z_{n}\right) E_{1 n} J_{1 n}\right]-o\left(n^{-1}\right) .
$$

Now, consider the further decomposition $J_{1 n}=J_{11 n}+J_{12 n}$, where $J_{11 n}:=\mathbb{1}_{A_{n} \cap F_{n} \cap\left\{E_{1 n} \geq 0\right\}}$ and $J_{12 n}:=\mathbb{1}_{A_{n} \cap F_{n} \cap\left\{E_{1 n}<0\right\}}$. Thus, concerning $J_{12 n}$ we have that

$$
\boldsymbol{E}\left[Z_{n}\left(1-Z_{n}\right) E_{1 n} J_{12 n}\right] \geq-\left(\frac{m_{2}}{y_{0}+c_{1}(n+1)}\right) \boldsymbol{P}\left(A_{n} \cap\left\{E_{1 n}<0\right\}\right) ;
$$


moreover, since $\boldsymbol{P}\left(Z_{n}<z_{\min }\right)$ and $\boldsymbol{P}\left(Z_{n}>z_{\max }\right)$ converge to zero with a sub-exponential rate by (4.3), in Theorem 4.1, we obtain

$$
\boldsymbol{E}\left[Z_{n}\left(1-Z_{n}\right) E_{1 n} J_{11 n}\right] \geq\left(\frac{z_{\min }\left(1-z_{\max }\right)\left(m_{1}-m_{2}\right)}{y_{0}+C_{1}(n+1)}\right) \boldsymbol{P}\left(A_{n} \cap\left\{E_{1 n}>0\right\}\right)-o\left(n^{-1}\right) .
$$

Therefore, from (4.18) we have

$$
I_{n} \geq n^{-1} c_{2} \boldsymbol{P}\left(A_{n}\right)-O\left(n^{-1}\right) \boldsymbol{P}\left(E_{1 n}<0\right)-o\left(n^{-1}\right),
$$

where $0<c_{2}<\infty$ is an appropriate constant. Hence, since from $m_{1}>m_{2}$ we have $\left\{E_{1 n}<0\right\} \equiv$ $R^{c}(\varepsilon, n)$, result (4.15) is obtained by establishing $\boldsymbol{P}\left(E_{1 n}<0\right) \rightarrow 0$. To this end, note that

$$
\boldsymbol{P}\left(E_{1 n}<0\right)=1-\boldsymbol{P}(R(\varepsilon, n)) \rightarrow 0,
$$

where $\boldsymbol{P}(R(\varepsilon, n)) \rightarrow 1$ follows from $\hat{\rho}_{1} \stackrel{p}{\rightarrow} \rho_{1}$, which is stated in (2.3) since $m_{1}>m_{2}$.

Let us recall that from (4.14) $Q^{+}(\varepsilon, n)=\left\{Z_{n}>\rho_{1}+\varepsilon\right\}$. We have the following result.

Lemma 4.4. Let $A_{n} \in \mathcal{F}_{n}$ be such that $A_{n} \subset Q^{+}(\varepsilon, n)$. Then, we have that

$$
\boldsymbol{E}\left[\left(Z_{n+1}-Z_{n}\right) \mathbb{1}_{A_{n}}\right] \leq-n^{-1} \cdot c_{2} \boldsymbol{P}\left(A_{n}\right)+o\left(n^{-1}\right) .
$$

Proof. The proof of this lemma is obtained by following analogous arguments of the proof of Lemma 4.3. In fact, we can first apply result (a) of Lemma 3.1, then note that

$$
\left\{Z_{n} \leq \hat{\rho}_{1, n}\right\} \subset\left(Q^{+c}(\varepsilon, n) \cup R^{c}(\varepsilon, n)\right),
$$

and

$$
\left\{Z_{n} \geq \hat{\rho}_{2, n}\right\} \supset\left(Q^{+}(\varepsilon, n) \cap R(\varepsilon, n)\right),
$$

where we recall that $R(\varepsilon, n):=\left\{\left|\hat{\rho}_{1, n}-\rho_{1}\right|<\varepsilon\right\}$. Hence, since $A_{n} \subset Q^{+}(\varepsilon, n)$, on the set $A_{n}$ we have that $W_{1, n} \leq \mathbb{1}_{R^{c}(\varepsilon, n)}$ and $W_{2, n} \geq \mathbb{1}_{R(\varepsilon, n)}$, which lead to the following inequality

$$
B_{n} \leq \boldsymbol{E}\left[\left(\frac{D_{1, n+1} \mathbb{1}_{R^{c}(\varepsilon, n)}}{Y_{n}+D_{1, n+1} \mathbb{1}_{R^{c}(\varepsilon, n)}}-\frac{D_{2, n+1} \mathbb{1}_{R(\varepsilon, n)}}{Y_{n}+D_{2, n+1} \mathbb{1}_{R(\varepsilon, n)}}\right) \mid \mathcal{F}_{n}\right]
$$

Then, by applying some standard calculations, we obtain that, on the set $A_{n}^{+}$,

$$
\begin{aligned}
B_{n} & \leq \boldsymbol{E}\left[\left(\frac{D_{1, n+1} \mathbb{1}_{R^{c}(\varepsilon, n)}}{Y_{n}}-\frac{D_{2, n+1} \mathbb{1}_{R(\varepsilon, n)}}{Y_{n}+b \mathbb{1}_{R(\varepsilon, n)}}\right) \mid \mathcal{F}_{n}\right] \\
& =\frac{m_{1} \mathbb{1}_{R^{c}(\varepsilon, n)}}{Y_{n}}-\frac{m_{2} \mathbb{1}_{R(\varepsilon, n)}}{Y_{n}+b \mathbb{1}_{R(\varepsilon, n)}} \\
& =\frac{m_{1} \mathbb{1}_{R^{c}(\varepsilon, n)}-m_{2} \mathbb{1}_{R(\varepsilon, n)}}{Y_{n}+b \mathbb{1}_{R(\varepsilon, n)}} .
\end{aligned}
$$


Now, we can go through the same previous calculations using the results of Theorem 4.1 and $\boldsymbol{P}\left(R^{c}(\varepsilon, n)\right) \rightarrow 0$, in order to prove (4.19).

Proof of Theorem 4.2. First, note that establishing (4.13) is equivalent to proving that for any $\mathcal{A}_{n} \in \mathcal{F}_{n}$ and letting $A_{n}:=\mathcal{A}_{n} \cap Q(\varepsilon, n)$ :

$$
\boldsymbol{E}\left[G\left(n, n^{-1}\right) \mathbb{1}_{A_{n}}\right] \leq-n^{-1} \cdot c_{2} \boldsymbol{P}\left(A_{n}\right)+o\left(n^{-1}\right),
$$

where we recall that $G\left(n, n^{-1}\right)=\left(\Delta_{n+1}-\Delta_{n}\right)$. Hence, consider $A_{n}^{+}:=A_{n} \cap Q^{+}(\varepsilon, n)$ and $A_{n}^{-}:=A_{n} \cap Q^{-}(\varepsilon, n)$. Since $A_{n}^{+} \cap A_{n}^{-}=\varnothing$ and $A_{n}^{+} \cup A_{n}^{-}=A_{n}$, we have the following decomposition

$$
\boldsymbol{E}\left[G\left(n, n^{-1}\right) \mathbb{1}_{A_{n}}\right]=I_{n}^{+}-I_{n}^{-},
$$

where

$$
I_{n}^{+}:=\boldsymbol{E}\left[\left(Z_{n+1}-Z_{n}\right) \mathbb{1}_{A_{n}^{+}}\right], \quad I_{n}^{-}:=\boldsymbol{E}\left[\left(Z_{n+1}-Z_{n}\right) \mathbb{1}_{A_{n}^{-}}\right] .
$$

By applying Lemmas 4.3 and 4.4 to $I_{n}^{-}$and $I_{n}^{+}$, respectively, we obtain

$$
\left\{\begin{array}{l}
I_{n}^{-} \geq n^{-1} \cdot c_{2} \boldsymbol{P}\left(A_{n}^{-}\right)-o\left(n^{-1}\right) \\
I_{n}^{+} \leq-n^{-1} \cdot c_{2} \boldsymbol{P}\left(A_{n}^{+}\right)+o\left(n^{-1}\right)
\end{array}\right.
$$

This concludes the proof.

\section{3. $L_{1}$ bound for $\Delta_{n}$ at linearly increasing times}

In this subsection, we provide an upper bound for the increments of $\Delta_{n}$ evaluated at linearly increasing times, i.e. $G(n, c)=\left(\Delta_{n+n c}-\Delta_{n}\right)$ and $c>0$, where we recall from (4.1) that $\Delta_{n}=$ $\left|\rho_{1}-Z_{n}\right|$. To this end, we claim that, for any fixed $\delta>0$, there exists a value $c>0$ such that for all $n \geq 0$

$$
\boldsymbol{P}\left(\left\{\left|Z_{n+n s_{\delta}}-Z_{n}\right|>\delta / 2\right\} \cap F_{n}\right)=0,
$$

where we recall from (4.12) that $F_{n}:=\left\{y_{0}+c_{1} n \leq Y_{n} \leq y_{0}+C_{1} n\right\}$. We will denote by $s_{\delta}$ one of these values of $c$.

We can compute precisely the range of values admissible for $s_{\delta}$ : on the set $F_{n}$, we obtain

$$
\left|Z_{n+n c}-Z_{n}\right| \leq b \sum_{i=n}^{n+n c} \frac{1}{Y_{i}} \leq \frac{b}{c_{1}} \sum_{i=n}^{n+n c} \frac{1}{i} \leq \frac{b}{c_{1}} \log (1+c),
$$

where we recall that $b$ is the maximum value of the urn reinforcements, i.e. $D_{1, n}, D_{2, n} \leq b$ a.s. for any $n \geq 1$. Then, imposing $\left|Z_{n+n c}-Z_{n}\right|<\delta / 2$, we obtain

$$
s_{\delta} \in\left(0, \exp \left(\frac{c_{1}}{2 b} \delta\right)-1\right) .
$$

This ensures that $\boldsymbol{P}\left(\left\{\left|Z_{n+n s_{\delta}}-Z_{n}\right|>\delta / 2\right\} \cap F_{n}\right)=0$ for all $n \geq 0$. 
The next theorem provides an $L_{1}$ upper bound for the difference $G\left(n, s_{\delta}\right)=\left(\Delta_{n+n s_{\delta}}-\Delta_{n}\right)$ on the set $Q(\delta, n)=\left\{\Delta_{n}>\delta\right\}$. An $L_{1}$ upper bound on the set $Q^{c}(\delta, n)$ is presented in Theorem 4.6.

Theorem 4.5. Let $m_{1}>m_{2},(2.2)$ and (2.3). Then, for any $\delta>0$ there exists a constant $0<C<$ $\infty$ such that

$$
\boldsymbol{E}\left[G\left(n, s_{\delta}\right) \mathbb{1}_{Q(\delta, n)}\right] \leq-C \boldsymbol{P}(Q(\delta, n))+o(1)
$$

Proof. First, note that using (4.4) in Theorem 4.1, we have

$$
\left|\boldsymbol{E}\left[G\left(n, s_{\delta}\right) \mathbb{1}_{Q(\delta, n) \cap F_{n}^{c}}\right]\right| \leq \boldsymbol{P}\left(F_{n}^{c}\right) \rightarrow 0 .
$$

Hence, define

$$
G_{n}:=\boldsymbol{E}\left[G\left(n, s_{\delta}\right) \mathbb{1}_{Q(\delta, n) \cap F_{n}}\right],
$$

and consider the following expression

$$
G_{n}=\sum_{i=n}^{n+n s_{\delta}-1} \boldsymbol{E}\left[G\left(i, i^{-1}\right) \mathbb{1}_{Q(\delta, n) \cap F_{n}}\right],
$$

where we recall that $G\left(i, i^{-1}\right)=\left(\Delta_{i+1}-\Delta_{i}\right)$. From the definition of $s_{\delta}$ in (4.20), on the set $F_{n}$ we have that for all $i \in\left\{n, \ldots, n+n s_{\delta}\right\}$

$$
Q(\delta, n) \subset Q(\delta / 2, i)
$$

where we recall that $Q(\delta, n)=\left\{\Delta_{n}>\delta\right\}$ and $Q(\delta / 2, i)=\left\{\Delta_{i}>\delta / 2\right\}$. Hence, by applying Theorem 4.2 to each term of the sum in (4.21), since $Q(\delta, n) \cap F_{n} \in \mathcal{F}_{i}$ for all $i \in\left\{n, \ldots, n+n s_{\delta}\right\}$, we obtain

$$
\begin{aligned}
\boldsymbol{E}\left[G\left(i, i^{-1}\right) \mathbb{1}_{Q(\delta, n) \cap F_{n}}\right] & =\boldsymbol{E}\left[\boldsymbol{E}\left[G\left(i, i^{-1}\right) \mathbb{1}_{Q(\delta / 2, i)} \mid \mathcal{F}_{i}\right] \mathbb{1}_{Q(\delta, n) \cap F_{n}}\right] \\
& \leq \boldsymbol{E}\left[\left(-i^{-1} \cdot c_{2} \mathbb{1}_{Q(\delta / 2, i)}+\psi_{i}\right) \mathbb{1}_{Q(\delta, n) \cap F_{n}}\right] \\
& =-i^{-1} \cdot c_{2} \boldsymbol{P}\left(Q(\delta, n) \cap F_{n}\right)+\boldsymbol{E}\left[\psi_{i} \mathbb{1}_{Q(\delta, n) \cap F_{n}}\right]
\end{aligned}
$$

Now, note that from (4.4) in Theorem 4.1 we have that $\boldsymbol{P}\left(Q(\delta, n) \cap F_{n}\right)=\boldsymbol{P}(Q(\delta, n))-o\left(i^{-1}\right)$; moreover, from Theorem 4.2, $\left|\boldsymbol{E}\left[\psi_{i} \mathbb{1}_{Q(\delta, n) \cap F_{n}}\right]\right| \leq \boldsymbol{E}\left[\left|\psi_{i}\right|\right]=o\left(i^{-1}\right)$. Thus, from (4.21) we have that

$$
\begin{aligned}
G_{n} & \leq-\sum_{i=n}^{n+n s_{\delta}-1} i^{-1} \cdot c_{2} \boldsymbol{P}(Q(\delta, n))+\sum_{i=n}^{n+n s_{\delta}-1} o\left(i^{-1}\right) \\
& \leq-\log \left(1+s_{\delta}\right) \cdot c_{2} \boldsymbol{P}(Q(\delta, n))+o(1) .
\end{aligned}
$$

The result follows after calling $C:=c_{2} \log \left(1+s_{\delta}\right)$. 
Now, we show that the expected difference $G\left(n, s_{\delta}\right)$ is asymptotically non-positive on the set $Q^{c}(\delta, n)$, for any $\delta>0$, where we recall that $G\left(n, s_{\delta}\right)=\left(\Delta_{n+n s_{\delta}}-\Delta_{n}\right), Q(\delta, n)=\left\{\Delta_{n}>\delta\right\}$ and $\Delta_{n}=\left|\rho_{1}-Z_{n}\right|$. The result is stated precisely in the following theorem.

Theorem 4.6. Let $m_{1}>m_{2},(2.2)$ and (2.3). Then, for any $\delta>0$,

$$
\varlimsup_{n} \boldsymbol{E}\left[G\left(n, s_{\delta}\right) \mathbb{1}_{Q^{c}(\delta, n)}\right] \leq 0 .
$$

To prove Theorem 4.6, we need to compare the ARRU model with two new urn models: $\left\{\tilde{Z}_{n}^{+} ; n \geq 1\right\}$ and $\left\{\tilde{Z}_{n}^{-} ; n \geq 1\right\}$. The dynamics of these processes is based on a sequence of random times $\left\{t_{n} ; n \geq 1\right\}$ which describes relation between the process $\left\{\Delta_{n} ; n \geq 1\right\}$ and an arbitrary fixed value $v>0$. Specifically, fix $v>0$ and, for any $n \geq 0$, define the set

$$
\mathcal{T}_{n}:=\left\{0 \leq k \leq n: Q^{c}(\nu, n-k)\right\},
$$

where we recall $Q^{c}(v, n-k)=\left\{\Delta_{n-k} \leq v\right\}$. Let $\left\{t_{n} ; n \geq 1\right\}$ be the sequence of random times defined as

$$
t_{n}= \begin{cases}\inf \left\{\mathcal{T}_{n}\right\} & \text { if } \mathcal{T}_{n} \neq \varnothing \\ \infty & \text { otherwise }\end{cases}
$$

The time $\left(n-t_{n}\right)$ indicates the last time up to $n$ the urn proportion is in the interval $\left(\rho_{1}-v\right.$, $\left.\rho_{1}+v\right)$.

First, let us describe the urn model $\left\{\tilde{Z}_{n}^{-} ; n \geq 1\right\}$. Let $\tilde{I}^{-}=1, \tilde{y}_{0} \in\left(0, y_{0}\right)$ and $\tilde{z}_{0}^{-} \in\left(0, \rho_{1}-v\right)$. The process $\left\{\tilde{Z}_{n}^{-} ; n \geq 1\right\}, \tilde{Z}_{n}^{-}=\tilde{Y}_{1, n} /\left(\tilde{Y}_{1, n}+\tilde{Y}_{2, n}\right)$, evolves as follows: if $t_{n-1}=0$, i.e. $\Delta_{n-1} \leq v$, or $t_{n-1}=\infty$, then $\tilde{X}_{n}=\mathbb{1}_{\left\{U_{n}<\tilde{z}_{0}^{-}\right\}}$and

$$
\left\{\begin{array}{l}
\tilde{Y}_{1, n}=\tilde{z}_{0}^{-} \cdot \tilde{y}_{0}+\tilde{X}_{n} D_{1, n} \tilde{I}^{-}, \\
\tilde{Y}_{2, n}=\left(1-\tilde{z}_{0}^{-}\right) \cdot \tilde{y}_{0}+\left(1-\tilde{X}_{n}\right) D_{2, n}
\end{array}\right.
$$

if $t_{n-1}=k \geq 1$, that is, $\Delta_{n-1}>v$, then $\tilde{X}_{n}=\mathbb{1}_{\left\{U_{n}<\tilde{Z}_{n-1}\right\}}$ and

$$
\left\{\begin{array}{l}
\tilde{Y}_{1, n}=\tilde{Y}_{1, n-1}+\tilde{X}_{n} D_{1, n} \tilde{I}^{-}, \\
\tilde{Y}_{2, n}=\tilde{Y}_{2, n-1}+\left(1-\tilde{X}_{n}\right) D_{2, n}
\end{array}\right.
$$

then, $\tilde{Y}_{n}:=\tilde{Y}_{1, n}+\tilde{Y}_{2, n}$ and $\tilde{Z}_{n}:=\tilde{Y}_{1, n} / \tilde{Y}_{n}$. The urn model is well defined since $t_{n-1}$ is $\mathcal{F}_{n-1^{-}}$ measurable.

Analogously, the urn model $\left\{\tilde{Z}_{n}^{+} ; n \geq 1\right\}, \tilde{Z}_{n}^{+}=\tilde{Y}_{1, n} /\left(\tilde{Y}_{1, n}+\tilde{Y}_{2, n}\right)$, is defined by the same equations (4.24) and (4.25), with $\tilde{I}^{-}$and $\tilde{z}_{0}^{-}$are replaced by $\tilde{I}^{+}=0$ and $\tilde{z}_{0}^{+} \in\left(\rho_{1}+v, 1\right)$, respectively.

In the next lemma, we state an important relation among the processes $\left\{\tilde{Z}_{n}^{-} ; n \geq 1\right\},\left\{\tilde{Z}_{n}^{+} ; n \geq\right.$ $1\}$ and the urn proportion of the ARRU model $\left\{Z_{n} ; n \geq 1\right\}$. This result is needed in the proof of Theorem 4.6. To ease calculations, let $h>0$ and fix the initial proportions $\tilde{z}_{0}^{-}$and $\tilde{z}_{0}^{+}$as follows:

$$
\rho_{1}-\tilde{z}_{0}^{-}=\tilde{z}_{0}^{+}-\rho_{1}=v+h .
$$


Let $M_{n}:=\sum_{i=n}^{n+n s_{\delta}} \mathbb{1}_{R^{c}(v, n)}$ and, for any $\epsilon>0$ define the set

$$
\mathcal{M}_{n}^{\epsilon}:=\left\{M_{n}<n s_{\delta} \epsilon\right\}
$$

where we recall that $R(v, n)=\left\{\left|\hat{\rho}_{1, n}-\rho_{1}\right| \leq v\right\}, s_{\delta}$ is such that $\boldsymbol{P}\left(\left\{\left|G\left(n, s_{\delta}\right)\right|>\delta / 2\right\} \cap F_{n}\right)=0$, with $F_{n}=\left\{y_{0}+c_{1} n \leq Y_{n} \leq y_{0}+C_{1} n\right\}$ from (4.12). Moreover, for any $n \geq 1$ and $k \in\{n, \ldots, n+$ $\left.n s_{\delta}\right\}$ let us define the set

$$
E(n, k):=\bigcup_{j=n}^{k} Q^{c}(\nu, j) \equiv\left\{\exists j \in\{n, \ldots, k\}:\left\{\Delta_{j} \leq \nu\right\}\right\} .
$$

We also introduce the following notation: $\tilde{\Delta}_{l}^{-}:=\left|\rho_{1}-\tilde{Z}_{l}^{-}\right|, \tilde{\Delta}_{l}^{+}:=\left|\rho_{1}-\tilde{Z}_{l}^{+}\right|$and $\tilde{\Delta}_{l}^{*}:=$ $\max \left\{\tilde{\Delta}_{l}^{-}, \tilde{\Delta}_{l}^{+}\right\}$. Thus, we have the following result:

Lemma 4.7. Let $m_{1}>m_{2},(2.2)$ and (2.3). Fix $n \geq 1, \tilde{y}_{0} \in\left(0, y_{0}+c_{1} n\right), \tilde{z}_{0}^{-}$and $\tilde{z}_{0}^{+}$as in (4.26). Consider the set $\mathcal{M}_{n}^{\epsilon}$ as defined in (4.27) with

$$
0<\epsilon<\frac{c_{1} h}{b s_{\delta}}
$$

Then, for any $n \geq 1$ and $l_{n} \in\left\{n+1, \ldots, n+n s_{\delta}\right\}$, on the set $\mathcal{M}_{n}^{\epsilon} \cap F_{n}$ we have that

$$
E\left(n, l_{n}\right) \subset Q^{c}\left(\tilde{\Delta}_{l}^{*}, l\right) \quad \text { a.s. }
$$

for all $l \in\left\{l_{n}+1, \ldots, n+n s_{\delta}\right\}$.

Proof. First, fix $l \in\left\{l_{n}+1, \ldots, n+n s_{\delta}\right\}$ and note that, from the definition of $\left\{t_{n} ; n \geq 1\right\}$ in (4.23) and $E(n, k)$ in (4.28), we always have

$$
\left\{t_{l-1}=\infty\right\} \cap E\left(n, l_{n}\right)=\varnothing .
$$

Hence, we never consider in this proof the set $\left\{t_{l-1}=\infty\right\}$.

Then, consider the set $\left\{t_{l-1}=0\right\}$ and note that, from the definition of $t_{n}$ in (4.23), $\left\{t_{l-1}=0\right\} \equiv$ $Q^{c}(v, l-1)$, which implies that, on the set $\left\{t_{l-1}=0\right\} \cap\left\{X_{l}=0\right\}$,

$$
Z_{l} \geq \frac{\left(\rho_{1}-v\right) Y_{l-1}}{Y_{l-1}+D_{2, l} W_{2, l-1}} \geq \frac{\tilde{z}_{0}^{-} \tilde{y}_{0}}{\tilde{y}_{0}+D_{2, l}}=\tilde{Z}_{l}^{-} \quad \text { a.s. }
$$

and, on the set $\left\{t_{l-1}=0\right\} \cap\left\{X_{l}=1\right\}$,

$$
Z_{l} \leq \frac{\left(\rho_{1}+v\right) Y_{l-1}+D_{1, l} W_{1, l-1}}{Y_{l-1}+D_{1, l} W_{1, l-1}} \leq \frac{\tilde{z}_{0}^{+} \tilde{y}_{0}+D_{1, l}}{\tilde{y}_{0}+D_{1, l}}=\tilde{Z}_{l}^{+} \quad \text { a.s. }
$$

From (4.31) and (4.32), we have $\tilde{Z}_{l}^{-} \leq Z_{l} \leq \tilde{Z}_{l}^{+}$a.s., that ensures that (4.30) is verified whenever $\left\{t_{l-1}=0\right\}$. 
To prove (4.30) on the set $\left\{1 \leq t_{l-1}<\infty\right\}$, we will show that, defining $\tilde{A}_{l}^{-}:=\left\{\tilde{Z}_{l}^{-} \leq Z_{l}\right\}$, $\tilde{A}_{l}^{+}:=\left\{Z_{l} \leq \tilde{Z}_{l}^{+}\right\}$and $B:=\mathcal{M}_{n}^{\epsilon} \cap F_{n} \cap\left\{1 \leq t_{l-1}<\infty\right\}$,

$$
\begin{aligned}
& \left(B \cap Q^{-}\left(v, l-t_{l-1}\right)\right) \subseteq\left(\tilde{A}_{l}^{-} \cap Q^{-}\left(v, l-t_{l-1}\right)\right), \\
& \left(B \cap Q^{+}\left(v, l-t_{l-1}\right)\right) \subseteq\left(\tilde{A}_{l}^{+} \cap Q^{+}\left(v, l-t_{l-1}\right)\right) .
\end{aligned}
$$

Moreover, from the definition of $\left\{t_{n} ; n \geq 1\right\}$ in (4.23), on the set $\left\{1 \leq t_{l-1}<\infty\right\}$, we note that

$$
\begin{aligned}
& \left\{X_{l-t_{l-1}}=0\right\} \equiv\left\{Z_{l-t_{l-1}} \leq \rho_{1}-v\right\}=Q^{-}\left(v, l-t_{l-1}\right), \\
& \left\{X_{l-t_{l-1}}=1\right\} \equiv\left\{Z_{l-t_{l-1}} \geq \rho_{1}+v\right\}=Q^{+}\left(v, l-t_{l-1}\right) .
\end{aligned}
$$

Hence, showing (4.33) is equivalent to establish the following

$$
\begin{aligned}
& \left(B \cap\left\{X_{l-t_{l-1}}=0\right\}\right) \subseteq\left(\tilde{A}_{l}^{-} \cap\left\{X_{l-t_{l-1}}=0\right\}\right), \\
& \left(B \cap\left\{X_{l-t_{l-1}}=1\right\}\right) \subseteq\left(\tilde{A}_{l}^{+} \cap\left\{X_{l-t_{l-1}}=1\right\}\right) .
\end{aligned}
$$

To this end, we will prove by induction on $j \in\left\{l-t_{l-1}+1, \ldots, l\right\}$ the following results:

$$
\begin{aligned}
& \left(B \cap\left\{X_{l-t_{l-1}}=0\right\}\right) \subseteq\left(\left(\bigcap_{i=l-t_{l-1}}^{j} \tilde{A}_{i}^{-}\right) \cap\left\{X_{l-t_{l-1}}=0\right\}\right), \\
& \left(B \cap\left\{X_{l-t_{l-1}}=1\right\}\right) \subseteq\left(\left(\bigcap_{i=l-t_{l-1}}^{j} \tilde{A}_{i}^{+}\right) \cap\left\{X_{l-t_{l-1}}=1\right\}\right) .
\end{aligned}
$$

First, note that by (4.31) it follows that condition (4.35) is verified for $j=l-t_{l-1}$. Hence, the result is achieved by establishing (4.35) for $j \in\left\{l-t_{l-1}+1, \ldots, l\right\}$, assuming that (4.35) holds for $(j-1)$.

To this end, consider

$$
Z_{j}=\frac{Z_{l-t_{l-1}-1} Y_{l-t_{l-1}-1}+\sum_{i=l-t_{l-1}}^{j} X_{i} D_{1, i} W_{1, i-1}}{Y_{l-t_{l-1}-1}+\sum_{i=l-t_{l}}^{j} X_{i} D_{1, i} W_{1, i-1}+\sum_{i=l-t_{l-1}}^{j}\left(1-X_{i}\right) D_{2, i} W_{2, i-1}} .
$$

Now, note that by (4.35) we have $X_{i}=\mathbb{1}_{\left\{U_{i}<Z_{i-1}\right\}} \geq \mathbb{1}_{\left\{U_{i}<\tilde{Z}_{i-1}^{-}\right\}}=\tilde{X}_{i}^{-}$for any $(i-1)=$ $l-t_{l-1}, \ldots,(j-1)$, and since $Z_{l-t_{l-1}-1} \geq \rho_{1}-v \geq \tilde{z}_{0}^{-}$we also have that $X_{l-t_{l-1}} \geq \tilde{X}_{l-t_{l-1}}$. Moreover, since $Y_{l-t_{l-1}-1} \geq \tilde{y}_{0}$ and $X_{l-t_{l-1}}=0$, it follows that

$$
Z_{j} \geq \frac{\left(\rho_{1}-v\right) \tilde{y}_{0}+\sum_{i=l-t_{l-1}+1}^{j} \tilde{X}_{i}^{-} D_{1, i} W_{1, i-1}}{\tilde{y}_{0}+\sum_{i=l-t_{l}+1}^{j} \tilde{X}_{i}^{-} D_{1, i} W_{1, i-1}+\sum_{i=l-t_{l-1}}^{j}\left(1-\tilde{X}_{i}^{-}\right) D_{2, i} W_{2, i-1}} .
$$

Note that, letting $n_{0}$ such that $\boldsymbol{P}\left(R\left(\nu, n_{0}\right)\right)>\eta>0$, for any $n \geq n_{0}$ we have the following relation

$$
\left\{Z_{n} \leq \hat{\rho}_{1, n}\right\} \supset\left(Q^{-}(v, n) \cap R(v, n)\right),
$$


where we recall that $R(v, n)=\left\{\left|\hat{\rho}_{1, n}-\rho_{1}\right|<v\right\}$ and $Q^{-}(v, n)=\left\{Z_{n}<\rho_{1}-v\right\}$. Hence, by definition of $t_{l-1}$ in (4.23), we have $Q^{-}(v, i)$ for any $i=l-t_{l-1}, \ldots, j-1$, and $\left\{Z_{i} \leq \hat{\rho}_{1, i}\right\} \supset$ $R(v, i)$, which implies $W_{1, i} \geq \mathbb{1}_{R(v, i)}$. Combining this argument with $W_{2, i} \leq 1$, we have that

$$
Z_{j} \geq \frac{\left(\rho_{1}-v\right) \tilde{y}_{0}+\sum_{i=l-t_{l-1}+1}^{j} \tilde{X}_{i}^{-} D_{1, i} \mathbb{1}_{R(v, i-1)}}{\tilde{y}_{0}+\sum_{i=l-t_{l-1}+1}^{j} \tilde{X}_{i}^{-} D_{1, i} \mathbb{1}_{R(v, i-1)}+\sum_{i=l-t_{l-1}}^{j}\left(1-\tilde{X}_{i}^{-}\right) D_{2, i}} .
$$

In addition, on the set $\mathcal{M}_{n}^{\epsilon}$ we have that

$$
\begin{aligned}
\sum_{i=l-t_{l-1}+1}^{j} \tilde{X}_{i}^{-} D_{1, i} \mathbb{1}_{R(v, i-1)} & \geq \sum_{i=l-t_{l-1}+1}^{j} \tilde{X}_{i}^{-} D_{1, i}-b M_{n} \\
& \geq \sum_{i=l-t_{l-1}+1}^{j} \tilde{X}_{i}^{-} D_{1, i}-n b s_{\delta} \epsilon
\end{aligned}
$$

Moreover, condition (4.29) ensures that

$$
\left(\rho_{1}-v\right) \tilde{y}_{0}-n b s_{\delta} \epsilon \geq \tilde{z}_{0}^{-} \tilde{y}_{0}
$$

which implies $\tilde{A}_{l}^{-}=\left\{Z_{j} \geq \tilde{Z}_{j}^{-}\right\}$.

Analogous arguments can be followed to establish (4.36) for any $j \in\left\{l-t_{l-1}+1, \ldots, l\right\}$. Finally, combining (4.35) and (4.36), we obtain (4.34). This concludes the proof.

In the next lemma, we show an important result required in the proof of Theorem 4.6, concerning the probability that $\tilde{Z}_{n}$ exceeds an arbitrary threshold $l>0$. This result is obtained by using comparison arguments between the process $\left\{\tilde{\Delta}_{n}^{*} ; n \geq 1\right\}$ and the urn proportion of an RRU model, where we recall that $\tilde{\Delta}_{n}^{*}=\max \left\{\tilde{\Delta}_{l}^{-}, \tilde{\Delta}_{l}^{+}\right\}, \tilde{\Delta}_{l}^{-}:=\left|\rho_{1}-\tilde{Z}_{l}^{-}\right|$and $\tilde{\Delta}_{l}^{+}:=\left|\rho_{1}-\tilde{Z}_{l}^{+}\right|$. The result is the following:

Lemma 4.8. Let $m_{1}>m_{2}$, and

$$
\tilde{T}_{n}:=\left\{k_{n}<t_{n}<\infty\right\}, \quad H_{n}:=\left\{\tilde{\Delta}_{n}^{*}>v\right\},
$$

where $\left\{k_{n} ; n \geq 1\right\}$ is a deterministic sequence such that $k_{n} \rightarrow \infty$. Fix $0<\tilde{y}_{0}<\infty$ and define $\tilde{z}_{0}^{-}$ and $\tilde{z}_{0}^{+}$as in (4.26). Then,

$$
\lim _{n \rightarrow \infty} \boldsymbol{P}\left(H_{n} \cup \tilde{T}_{n}\right)=0 .
$$

Proof. Since $H_{n}=H_{n}^{-} \cup H_{n}^{+}$where

$$
H_{n}^{-}:=\left\{\tilde{Z}_{n}^{-}<\rho_{1}-v\right\} \text { and } H_{n}^{+}:=\left\{\tilde{Z}_{n}^{+}>\rho_{1}+v\right\},
$$


equation (4.38) is established by proving

$$
\lim _{n \rightarrow \infty} \boldsymbol{P}\left(H_{n}^{-} \cup \tilde{T}_{n}\right)+\boldsymbol{P}\left(H_{n}^{+} \cup \tilde{T}_{n}\right)=0 .
$$

We will show that $\boldsymbol{P}\left(H_{n}^{-} \cup \tilde{T}_{n}\right) \rightarrow 0$, since the proof of $\boldsymbol{P}\left(H_{n}^{+} \cup \tilde{T}_{n}\right) \rightarrow 0$ is analogous.

First, we recall that $t_{n}$, defined in (4.23), satisfies that $Q^{c}\left(v, n-t_{n}\right)=\left\{\Delta_{n-t_{n}} \leq v\right\}$ and when $t_{n}>0, Q(v, i)=\left\{\Delta_{i}>v\right\}$ for any $n-t_{n}<i \leq n$. Hence, on the set $\tilde{T}_{n}$ the process $\tilde{Z}_{i}^{-}$evolves at times $n-t_{n}<i \leq n$ as described in (4.25), yielding $\tilde{X}_{i}=\mathbb{1}_{\left\{U_{i}<\tilde{Z}_{i-1}^{-}\right\}}$and

$$
\left\{\begin{array}{l}
\tilde{Y}_{1, n}^{-}=\tilde{z}_{0}^{-} \tilde{y}_{0}+\sum_{i=n-t_{n}+1}^{n} \tilde{X}_{i} D_{1, i} \\
\tilde{Y}_{2, n}^{-}=\left(1-\tilde{z}_{0}^{-}\right) \tilde{y}_{0}+\sum_{i=n-t_{n}+1}^{n}\left(1-\tilde{X}_{i}\right) D_{2, i}
\end{array}\right.
$$

Now, consider an RRU model $\left\{Z_{j}^{R} ; j \geq 1\right\}$ with initial composition $\left(\tilde{y}_{1,0}^{R}, \tilde{y}_{2,0}^{R}\right)=\left(\tilde{z}_{0}^{-} \tilde{y}_{0},(1-\right.$ $\left.\left.\tilde{z}_{0}^{-}\right) \tilde{y}_{0}\right)$; the reinforcements are defined as $D_{1, j}^{R}=D_{1, n-t_{n}+j}$ and $D_{2, j}^{R}=D_{2, n-t_{n}+j}$ for any $i \geq 1$ a.s.; the sampling process is modeled by $X_{j}^{R}:=\mathbb{1}_{\left\{U_{j}^{R}<Z_{j-1}^{R}\right\}}$ and $U_{j}^{R}=U_{n-t_{n}+j}$ a.s., Hence, the composition of the RRU model at time $j \geq 1$ can be expressed as follows:

$$
\begin{aligned}
Y_{1, j}^{R} & =\tilde{y}_{1,0}^{R}+\sum_{i=1}^{j} X_{n-t_{n}+i} D_{1, n-t_{n}+i} \\
& =\tilde{z}_{0}^{-} \tilde{y}_{0}^{-}+\sum_{i=n-t_{n}+1}^{n-t_{n}+j} X_{i} D_{1, i}, \\
Y_{2, j}^{R} & =\tilde{y}_{2,0}^{R}+\sum_{i=1}^{j}\left(1-X_{n-t_{n}+i}\right) D_{2, n-t_{n}+i} \\
& =\left(1-\tilde{z}_{0}^{-}\right) \tilde{y}_{0}+\sum_{i=n-t_{n}+1}^{n-t_{n}+j}\left(1-X_{i}\right) D_{2, i} .
\end{aligned}
$$

Hence, combining (4.39) and (4.40) with $j=t_{n}$, we have that on the set $\tilde{T}_{n}$

$$
\left(\tilde{Y}_{1, n}^{-}, \tilde{Y}_{2, n}^{-}\right)=\left(Y_{1, t_{n}}^{R}, Y_{2, t_{n}}^{R}\right)
$$

Now, from the asymptotic behavior of the RRU studied in Muliere, Paganoni and Secchi [22], Theorem 8, we have that (since $\left.m_{1}>m_{2}\right) \boldsymbol{P}\left(\lim _{n \rightarrow \infty} Z_{n}^{R}=1\right)=1$. Thus, on the set $\tilde{T}_{n}$ we have $\left\{\lim _{n \rightarrow \infty} Z_{n}^{R}=1\right\}$, which implies $\boldsymbol{P}\left(H_{n}^{-} \cup \tilde{T}_{n}\right) \rightarrow 0$. This concludes the proof. 
Proof of Theorem 4.6. First, consider the set $F_{n}=\left\{y_{0}+c_{1} n \leq Y_{n} \leq y_{0}+C_{1} n\right\}$ defined in (4.12) and by using (4.4) in Theorem 4.1 we have

$$
\varlimsup_{n} \boldsymbol{P}\left(F_{n}^{c}\right)=0 .
$$

Hence, since $\left|G\left(n, s_{\delta}\right)\right| \leq \max \left\{Z_{n+n s_{\delta}} ; Z_{n}\right\}<1$ a.s., to prove (4.22) it is enough to show that for any $0<h<1 / 2$

$$
\boldsymbol{E}\left[G_{n, s_{\delta}} \mathbb{1}_{Q^{c}(\delta, n) \cap F_{n}}\right] \leq h+o(1),
$$

where we recall that $G\left(n, s_{\delta}\right)=\left(\Delta_{n+n s_{\delta}}-\Delta_{n}\right)$ and $Q(\delta, n)=\left\{\Delta_{n}>\delta\right\}$. Now, define $H:=[\delta / h]$ and note that

$$
[0, \delta] \subset[0,(H+1) h]=\bigcup_{i=0}^{H}[i h,(i+1) h]
$$

then, calling

$$
\bar{Q}((i+1) h, n):=Q^{c}((i+1) h, n) \backslash Q^{c}(i h, n)=\left\{i h<\Delta_{n}<(i+1) h\right\}
$$

(where for any two sets $A$ and $B, A \backslash B=A \cap B^{c}$ ), we have $Q^{c}(\delta, n)=\bigcup_{i=0}^{H} \bar{Q}((i+1) h, n)$ and hence the left-hand side of (4.41) can be written as

$$
\boldsymbol{E}\left[G\left(n, s_{\delta}\right) \mathbb{1}_{Q^{c}(\delta, n) \cap F_{n}}\right]=\sum_{i=0}^{H} \boldsymbol{E}\left[G\left(n, s_{\delta}\right) \mathbb{1}_{\bar{Q}((i+1) h, n) \cap F_{n}}\right]
$$

thus, result (4.41) can be achieved by establishing the following

$$
\boldsymbol{E}\left[G\left(n, s_{\delta}\right) \mathbb{1}_{\bar{Q}((i+1) h, n) \cap F_{n}}\right] \leq h \cdot \boldsymbol{P}(\bar{Q}((i+1) h, n))+o(1),
$$

for any $i \in\{1, \ldots, H\}$. Now, fix $i \in\{0, \ldots, H\}$, call $v:=(i+1) h$ and consider the set $\mathcal{M}_{n}^{\epsilon}:=$ $\left\{M_{n}<n s_{\delta} \epsilon\right\}$ defined in (4.27), where we recall that $M_{n}=\sum_{i=n}^{n+n s_{\delta}} \mathbb{1}_{R^{c}(\nu, n)}$. The left-hand side of (4.42) can be so decomposed $\boldsymbol{E}\left[G\left(n, s_{\delta}\right) \mathbb{1}_{\bar{Q}}(v, n) \cap F_{n}\right]=\mathcal{G}_{1 n}+\mathcal{G}_{2 n}$, where

$$
\mathcal{G}_{1 n}:=\boldsymbol{E}\left[G\left(n, s_{\delta}\right) \mathbb{1}_{\bar{Q}(v, n) \cap F_{n} \cap \mathcal{M}_{n}^{\epsilon}}\right] \quad \text { and } \quad \mathcal{G}_{2 n}:=\boldsymbol{E}\left[G\left(n, s_{\delta}\right) \mathbb{1}_{\bar{Q}(v, n) \cap F_{n} \cap \mathcal{M}_{n}^{\epsilon c}}\right] .
$$

Since $\boldsymbol{P}(R(v, n)) \rightarrow 1$ from (2.3), and by using Markov's inequality we have that

$$
\boldsymbol{P}\left(\mathcal{M}_{n}^{\epsilon c}\right) \leq \epsilon^{-1} \frac{1}{n s_{\delta}} \sum_{i=n}^{n+n s_{\delta}} \boldsymbol{P}\left(R^{c}(v, n)\right) \rightarrow 0
$$

thus, since $\left|G\left(n, s_{\delta}\right)\right| \leq \max \left\{Z_{n+n s_{\delta}} ; Z_{n}\right\}<1$ a.s., we have $\mathcal{G}_{2 n} \rightarrow 0$ and hence result (4.42) can be achieved by establishing the following

$$
\mathcal{G}_{1 n}=\boldsymbol{E}\left[G\left(n, s_{\delta}\right) \mathbb{1}_{\bar{Q}(v, n) \cap F_{n} \cap \mathcal{M}_{n}^{\epsilon}}\right] \leq h \cdot \boldsymbol{P}(\bar{Q}(v, n))+o(1),
$$

where we recall that $\bar{Q}(v, n)=\left\{v-h<\Delta_{n}<v\right\}$. 
Now, following the same arguments used to determine $s_{\delta}$ in (4.20), we can fix a value $s_{h}$ such that

$$
\boldsymbol{P}\left(\left\{\left|G\left(n, s_{h}\right)\right|>h / 2\right\} \cap F_{n}\right)=0,
$$

where we recall that $G\left(n, s_{h}\right)=\left(\Delta_{n+n s_{h}}-\Delta_{n}\right)$. Analogously to (4.20), the range of values admissible for $s_{h}$ is

$$
s_{h} \in\left(0, \exp \left(\frac{c_{1}}{2 b} h\right)-1\right),
$$

where we recall that $c_{1}>0$ is a constant introduce in (4.12) to define $F_{n}$.

Now, consider the random time $t_{j}$ defined in (4.23) as the smallest time $k$ such that $Q^{c}(v, n-k)$ occurs, i.e. $n-t_{n}$ indicates the last time up to $n$ the urn proportion is in the interval $\left(\rho_{1}-v, \rho_{1}+v\right)$. Then, call $\tau_{n}:=t_{n+n s_{\delta}}$ and note that, since $\bar{Q}(v, n) \subset Q^{c}(v, n)$ by definition of $\bar{Q}(v, n)$, we have that

$$
\boldsymbol{P}\left(\tau_{n} \leq n s_{\delta} \mid \bar{Q}(v, n)\right)=1 .
$$

Hence, define $S_{H}:=\left[s_{\delta} / s_{h}\right]$ and, assuming wlog that $s_{\delta}=S_{H} s_{h} h+1$, on the set $\bar{Q}(v, n)$, consider the partition $\left\{0, \ldots, n s_{\delta}\right\}=\bigcup_{k=0}^{S_{H}} \mathcal{T}_{k}^{n}$, where $\mathcal{T}_{k}^{n}:=\left\{n k s_{h}, \ldots, n(k+1) s_{h}\right\}$; thus, the lefthand side of (4.43) can be decomposed as $\mathcal{G}_{1 n}=\sum_{k=0}^{S_{H}} T_{k}^{n}$, where for any $k \in\left\{0, \ldots, S_{H}\right\}$

$$
T_{k}^{n}:=\boldsymbol{E}\left[G\left(n, s_{\delta}\right) \mathbb{1}_{\bar{Q}(v, n) \cap F_{n} \cap \mathcal{M}_{n}^{\epsilon} \cap\left\{\tau_{n} \in \mathcal{T}_{k}^{n}\right\}}\right]
$$

Hence, equation (4.43) can be achieved by establishing the following

$$
T_{k}^{n} \leq h \cdot \boldsymbol{P}\left(\bar{Q}(v, n) \cap\left\{\tau_{n} \in \mathcal{T}_{k}^{n}\right\}\right)+o(1), \quad \forall k \in\left\{0, \ldots, S_{H}\right\}
$$

First, consider $k=0$ in (4.45). From the definition of $\tau_{n}$, we have

$$
\left\{\tau_{n} \in \mathcal{T}_{0}^{n}\right\} \subset Q^{c}\left(v+h, n+n s_{\delta}\right),
$$

where we recall that $Q^{c}\left(v+h, n+n s_{\delta}\right)=\left\{\Delta_{n+n s_{\delta}}<v+h\right\}$. Hence, using (4.46) in (4.44), it is immediate to obtain (4.45).

For $k \in\left\{1, \ldots, S_{H}\right\}$ in (4.45), from the definition of $\tau_{n}$ and $E_{n, k}$ in (4.28), we have that

$$
\left\{\tau_{n} \in \mathcal{T}_{k}^{n}\right\} \subset E\left(n, n+n\left(s_{\delta}-k s_{h}\right)\right),
$$

where we recall $E(n, k)=\bigcup_{j=n}^{k} Q^{c}(v, j)$. Hence, we can use Lemma 4.7 with $l_{n}=n+n\left(s_{\delta}-\right.$ $\left.k s_{h}\right)$, to obtain, on the set $\mathcal{M}_{n}^{\epsilon} \cap F_{n}$, for any $j \in\left\{n+n\left(s_{\delta}-k s_{h}\right)+1, \ldots, n+n s_{\delta}\right\}$

$$
Q^{c}\left(v, n+n\left(s_{\delta}-k s_{h}\right)\right) \subset Q^{c}\left(\tilde{\Delta}_{j}^{*}, j\right) \quad \text { a.s. }
$$

where we recall that $Q^{c}(\nu, j)=\left\{\Delta_{j}<v\right\}$ and $Q^{c}\left(\tilde{\Delta}_{j}^{*}, j\right)=\left\{\Delta_{j}<\tilde{\Delta}_{j}^{*}\right\}, \tilde{\Delta}_{j}^{*}=\max \left\{\tilde{\Delta}_{j}^{-}, \tilde{\Delta}_{j}^{+}\right\}$, $\tilde{\Delta}_{j}^{-}=\left|\rho_{1}-\tilde{Z}_{j}^{-}\right|$and $\tilde{\Delta}_{j}^{+}=\left|\rho_{1}-\tilde{Z}_{j}^{+}\right|$. In particular, by using (4.47) and since $\bar{Q}(v, n) \subset Q(v-$ $h, n)=\left\{\Delta_{n}>v-h\right\}$, from (4.44) we obtain

$$
T_{k}^{n} \leq \boldsymbol{E}\left[\left(\tilde{\Delta}_{n+n s_{\delta}}^{*}-v+h\right) \mathbb{1}_{\bar{Q}(v, n) \cap F_{n} \cap \mathcal{M}_{n}^{\epsilon} \cap\left\{\tau_{n} \in \mathcal{T}_{k}^{n}\right\}}\right] .
$$


Note that, from the definition of $\tau_{n}$ and $\mathcal{T}_{k}^{n}$, we have

$$
\left\{\tau_{n} \in \mathcal{T}_{k}^{n}\right\} \subset\left\{n k s_{h}<t_{n+n s_{\delta}}<n(k+1) s_{h}\right\} .
$$

Hence, we can apply Lemma 4.8 with $k_{n+n s_{\delta}}=n k s_{h}, \tilde{T}_{j}:=\left\{\tilde{\Delta}_{j}^{*}>v\right\}$ and $H_{j}:=\left\{k_{j}<t_{j}<\infty\right\}$ as defined in (4.37), so obtaining

$$
\boldsymbol{E}\left[\left(\tilde{\Delta}_{n+n s_{\delta}}^{*}-v\right)^{+} \mathbb{1}_{\left\{\tau_{n} \in \mathcal{T}_{k}^{n}\right\}}\right] \leq \boldsymbol{P}\left(H_{n+n s_{\delta}} \cup \tilde{T}_{n+n s_{\delta}}\right) \rightarrow 0 .
$$

Hence, applying these results to (4.48), we obtain

$$
T_{k}^{n} \leq h \cdot \boldsymbol{P}\left(\bar{Q}(v, n) \cap\left\{\tau_{n} \in \mathcal{T}_{k}^{n}\right\}\right)+o(1),
$$

that corresponds to (4.45). This concludes the proof.

\subsection{Proof of weak consistency}

Proof of Theorem 2.2. The result is established by proving that, for any $l>0$ and any $\epsilon>0$, there exists $n_{0} \in \mathbb{N}$ such that

$$
\boldsymbol{P}(Q(l, n))<\epsilon,
$$

for any $n \geq n_{0}$, where we recall that $Q(l, n)=\left\{\Delta_{n}>l\right\}$ and $\Delta_{n}=\left|\rho_{1}-Z_{n}\right|$. To this end, fix $0<\epsilon^{\prime}<\frac{l \epsilon}{3}$ and $0<\delta<\epsilon^{\prime}$ to define the conditions

$$
\mathcal{A}_{n}:=\left\{\boldsymbol{P}(Q(\delta, n))<\epsilon^{\prime}\right\}, \quad \mathcal{B}_{n}:=\left\{\boldsymbol{E}\left[\Delta_{n}\right]<2 \epsilon^{\prime}\right\} .
$$

It is immediate to see that $\mathcal{B}_{n}$ implies (4.49). Thus, (4.49) can be established by proving that

(a) for any $N \geq 1$ there exists $n_{0} \geq N$ such that $\mathcal{A}_{n_{0}}$ occurs;

(b) there exists $n_{0} \geq 1$ such that for any $n \geq n_{0}, \mathcal{A}_{n} \subset \mathcal{B}_{k}$ for all $k \in\left\{n+1, \ldots, n\left(1+s_{\delta}\right)\right\}$;

(c) there exists $n_{0} \geq 1$ such that for any $n \geq n_{0}, \mathcal{B}_{n} \subset \mathcal{B}_{k}$ for all $k \in\left\{n\left(1+s_{\delta}\right), \ldots,(n+\right.$ 1) $\left.\left(1+s_{\delta}\right)\right\}$.

For part (a), we will show that there cannot exist $N \geq 1$ such that

$$
\mathcal{A}_{n}^{c}:=\left\{\boldsymbol{P}(Q(\delta, n)) \geq \epsilon^{\prime}\right\}
$$

occurs for all $n \geq N$. First, we combine Theorem 4.5 and Theorem 4.6 to obtain

$$
\boldsymbol{E}\left[G\left(n, s_{\delta}\right)\right] \leq-C\left(\boldsymbol{P}(Q(\delta, n))-\frac{\epsilon^{\prime}}{2}\right),
$$

with $0<C<\infty$, where we recall that $G\left(n, s_{\delta}\right)=\left(\Delta_{n+n s_{\delta}}-\Delta_{n}\right)$. Now, if (4.50) holds, then there exists a subsequence $\left\{k_{n} ; n \geq 1\right\}$ such that, $k_{1}=N$ and $k_{n}=k_{n-1}\left(1+s_{\delta}\right)$ for all $n \geq 2$, and by $(4.51)$

$$
\boldsymbol{E}\left[\Delta_{k_{n}}\right]=\sum_{i=1}^{n} \boldsymbol{E}\left[G\left(k_{i-1}, s_{\delta}\right)\right] \leq-\sum_{i=1}^{n} C \frac{\epsilon^{\prime}}{2}=-\infty
$$


where $G\left(k_{i-1}, s_{\delta}\right)=\left(\Delta_{k_{i}}-\Delta_{k_{i-1}}\right)$, which is a contradiction and hence part (a) holds. For part (b), consider the time $n$ at which $\mathcal{A}_{n}$ occurs. Fix $k \in\left\{n+1, \ldots, n+n s_{\delta}\right\}$ and note that $\boldsymbol{E}\left[\Delta_{k}\right] \leq$ $J_{1 n}+J_{2 n, k}$ where

$$
J_{1 n}:=\boldsymbol{E}\left[\Delta_{n}\right] \quad \text { and } \quad J_{2 n, k}:=\boldsymbol{E}\left[\left|\Delta_{k}-\Delta_{n}\right|\right] .
$$

From definition of $s_{\delta}$ in (4.20), we have

$$
\begin{aligned}
J_{2 n, k} & \leq \boldsymbol{E}\left[\left|\Delta_{k}-\Delta_{n}\right| \mathbb{1}_{F_{n}}\right]+\boldsymbol{E}\left[\left|\Delta_{k}-\Delta_{n}\right| \mathbb{1}_{F_{n}^{c}}\right] \\
& \leq \delta+\boldsymbol{P}\left(F_{n}^{c}\right),
\end{aligned}
$$

and using $\boldsymbol{P}\left(F_{n}^{c}\right) \rightarrow 0$ from (4.4) in Theorem 4.1 we have that $\lim _{n \rightarrow \infty} J_{2 n, k} \leq \delta$. Thus, there exists $n_{0} \geq 1$ such that $J_{2 n, k}<2 \delta$ for any $n \geq n_{0}$. Then, note that $J_{1 n}=J_{3 n}+J_{4 n}$ where

$$
J_{3 n}:=\boldsymbol{E}\left[\Delta_{n} \mathbb{1}_{Q^{c}(\delta, n)}\right] \quad \text { and } \quad J_{4 n}:=\boldsymbol{E}\left[\Delta_{n} \mathbb{1}_{Q(\delta, n)}\right] .
$$

Notice that $J_{3 n} \leq \delta \boldsymbol{P}\left(Q^{c}(\delta, n)\right)<\delta$ and $J_{4 n} \leq \boldsymbol{P}(Q(\delta, n))<\epsilon^{\prime}$, and hence we have $J_{1 n}<\delta+\epsilon^{\prime}$. Thus, combining $J_{1 n}$ and $J_{2 n}$, since $\delta<\epsilon^{\prime} / 3$, we obtain for any $n \geq n_{0}$

$$
\boldsymbol{E}\left[\Delta_{k}\right] \leq J_{1 n}+J_{2 n, k}<\delta+\epsilon^{\prime}+2 \delta<2 \epsilon^{\prime},
$$

that implies (b). For part (c), for any $k \in\left\{n\left(1+s_{\delta}\right), \ldots,(n+1)\left(1+s_{\delta}\right)\right\}$ consider

$$
\boldsymbol{E}\left[\left|\Delta_{k}-\Delta_{n+n s_{\delta}}\right|\right] \leq \boldsymbol{E}\left[\left|\Delta_{k}-\Delta_{n+n s_{\delta}}\right| \mathbb{1}_{F_{n}}\right]+\boldsymbol{E}\left[\left|\Delta_{k}-\Delta_{n+n s_{\delta}}\right| \mathbb{1}_{F_{n}^{c}}\right] .
$$

First, note that $\boldsymbol{P}\left(F_{n}^{c}\right) \rightarrow 0$ from (4.4) in Theorem 4.1. Then, since $\left|k-\left(n+n s_{\delta}\right)\right| \leq\left(1+s_{\delta}\right)$ and $\left|Z_{n+1}-Z_{n}\right|<b / Y_{n}$ a.s., we have that

$$
\boldsymbol{P}\left(\left\{\left|Z_{k}-Z_{n+n s_{\delta}}\right|>\left(\frac{b}{y_{0}+c_{1} n}\right)\left(1+s_{\delta}\right)\right\} \cap F_{n}\right)=0 .
$$

Thus, for any $k \in\left\{n\left(1+s_{\delta}\right), \ldots,(n+1)\left(1+s_{\delta}\right)\right\}$ we have

$$
\boldsymbol{E}\left[\left|\Delta_{k}-\Delta_{n+n s_{\delta}}\right|\right] \leq\left(\frac{b\left(1+s_{\delta}\right)}{y_{0}+c_{1} n}\right)+\boldsymbol{P}\left(F_{n}^{c}\right) \rightarrow 0 .
$$

Now, since $\mathcal{B}_{n} \subset \mathcal{A}_{n} \cup \mathcal{C}_{n}$, where $\mathcal{C}_{n}=\left(\mathcal{B}_{n} \cap \mathcal{A}_{n}^{c}\right)$, part (c) is established by proving that there exists $n_{0} \geq 1$ such that, for any $n \geq n_{0}$,

(c1) $\mathcal{A}_{n} \subset \mathcal{B}_{k}$ for all $k \in\left\{n\left(1+s_{\delta}\right), \ldots,(n+1)\left(1+s_{\delta}\right)\right\}$;

(c2) $\mathcal{C}_{n} \subset \mathcal{B}_{k}$ for all $k \in\left\{n\left(1+s_{\delta}\right), \ldots,(n+1)\left(1+s_{\delta}\right)\right\}$.

For part (c1), we can follow the same arguments of part (b), except for $J_{2 n, k}$ since here $k \in$ $\left\{n\left(1+s_{\delta}\right), \ldots,(n+1)\left(1+s_{\delta}\right)\right\}$ and hence

$$
\begin{aligned}
J_{2 n, k} & \leq \boldsymbol{E}\left[\left|\Delta_{k}-\Delta_{n}\right| \mathbb{1}_{F_{n}}\right]+\boldsymbol{E}\left[\left|\Delta_{k}-\Delta_{n}\right| \mathbb{1}_{F_{n}^{c}}\right] \\
& \leq \boldsymbol{E}\left[\left|\Delta_{k}-\Delta_{n+n s_{\delta}}\right| \mathbb{1}_{F_{n}}\right]+\boldsymbol{E}\left[\left|\Delta_{n+n s_{\delta}}-\Delta_{n}\right| \mathbb{1}_{F_{n}}\right]+\boldsymbol{P}\left(F_{n}^{c}\right) \\
& \leq \boldsymbol{E}\left[\left|\Delta_{k}-\Delta_{n+n s_{\delta}}\right| \mathbb{1}_{F_{n}}\right]+\delta+\boldsymbol{P}\left(F_{n}^{c}\right)
\end{aligned}
$$


However, by using (4.52), we still have $\lim _{n \rightarrow \infty} J_{2 n, k} \leq \delta$ and so, analogously to part (b), there exists $n_{0} \geq 1$ such that $J_{n 2}<2 \delta$ for any $n \geq n_{0}$. Since $J_{1 n}$ does not depend on $k$, (c1) follows. For part (c2), we combine (4.51) and $\mathcal{A}_{n}^{c}$ to obtain

$$
\boldsymbol{E}\left[G\left(n, s_{\delta}\right)\right] \leq-C \frac{\epsilon^{\prime}}{2},
$$

where we recall that $G\left(n, s_{\delta}\right)=\left(\Delta_{n+n s_{\delta}}-\Delta_{n}\right)$. Moreover, by (4.52) there exists $n_{0} \geq 1$ such that $\boldsymbol{E}\left[\left|\Delta_{k}-\Delta_{n+n s_{\delta}}\right|\right] \leq C \frac{\epsilon^{\prime}}{2}$ for any $n \geq n_{0}$. Hence, (c2) follows by combining (4.52), (4.53) and $\mathcal{B}_{n}$ as follows:

$$
\boldsymbol{E}\left[\Delta_{k}\right] \leq \boldsymbol{E}\left[\left|\Delta_{k}-\Delta_{n+n s_{\delta}}\right|\right]+\boldsymbol{E}\left[G\left(n, s_{\delta}\right)\right]+\boldsymbol{E}\left[\Delta_{n}\right]=2 \epsilon^{\prime} .
$$

Remark 4.9. It is possible to present a modification of the current arguments along the traditional probabilistic lines. We chose to present the above alternative logical argument.

Remark 4.10. An anonymous referee raised the issue of relaxing the hypothesis concerning the boundedness of $u(\cdot)$. While such condition has been used in several estimates, we notice that it is not required in the proof of the Theorem 4.1. In this case, under weak additional conditions on the tails of $u\left(\xi_{1,1}\right)$ and $u\left(\xi_{2,1}\right)$ one can modify the arguments to obtain an analogous version of Theorem 4.1. The challenge however is to establish the comparison arguments between various urns without this hypothesis. This seems to be a challenging task at this moment even though the authors believe that the results should hold without the boundedness assumption. It is worth pointing out that even for the MRRU model, the limit theorems without the boundedness condition are not known.

\section{Proof of strong consistency}

In this section, we provide the proof of the strong consistency of the urn proportion $Z_{n}$ for any values of $m_{1}$ and $m_{2}$, when the random thresholds $\hat{\rho}_{1, n}$ and $\hat{\rho}_{2, n}$ converge with probability one.

Proof of Theorem 2.4. We divide the proof into three steps:

(a) $\boldsymbol{P}\left(\rho_{2} \leq \underline{\lim }_{n} Z_{n} \leq \varlimsup_{\lim _{n}} Z_{n} \leq \rho_{1}\right)=1$,

(b)

$$
\begin{cases}\boldsymbol{P}\left(\overline{\lim }_{n} Z_{n} \geq \rho_{1}\right)=1 & \text { if } m_{1}>m_{2}, \\ \boldsymbol{P}\left(\underline{\lim }_{n} Z_{n} \leq \rho_{2}\right)=1 & \text { if } m_{1}<m_{2},\end{cases}
$$

(c) $\boldsymbol{P}\left(\lim _{n} Z_{n}\right.$ exists $)=1$.

For part (a), firstly note that, when $\rho_{1}=1$ and $\rho_{2}=0$, result (a) is trivially true, hence consider $0<\rho_{2} \leq \rho_{1}<1$. We show that $\boldsymbol{P}\left(\overline{\lim }_{n} Z_{n} \leq \rho_{1}\right)=1$, since the proof of $\boldsymbol{P}\left(\lim _{n} Z_{n} \geq \rho_{2}\right)=1$ is completely analogous. To this end, we show that there cannot exist $\epsilon>0$ and $\rho^{\prime}>\rho_{1}$ such that

$$
\boldsymbol{P}\left(\overline{\lim }_{n} Z_{n}>\rho_{1}^{\prime}\right) \geq \epsilon>0 .
$$


We prove this by contradiction using a comparison argument with an RRU model. The proof involves last exit time arguments. Now, suppose (5.1) holds and let $A_{1}:=\left\{\overline{\lim }_{n} Z_{n}>\rho_{1}^{\prime}\right\}$. Let

$$
R_{1}:=\left\{k \geq 0: \hat{\rho}_{1, k} \geq \frac{\rho_{1}^{\prime}+\rho_{1}}{2}\right\},
$$

and denote the last time the process $\left\{\hat{\rho}_{1, n} ; n \geq 1\right\}$ is above $\left(\rho_{1}^{\prime}+\rho_{1}\right) / 2$ by

$$
t_{\frac{\rho_{1}^{\prime}+\rho_{1}}{2}}= \begin{cases}\sup \left\{R_{1}\right\} & \text { if } R_{1} \neq \varnothing ; \\ 0 & \text { otherwise. }\end{cases}
$$

Since $\hat{\rho}_{1, n} \stackrel{\text { a.s. }}{\rightarrow} \rho_{1}$ by $(2.5)$, then we have that $\boldsymbol{P}\left(\frac{t_{\rho_{1}^{\prime}+\rho_{1}}}{2}<\infty\right)=1$. Hence, there exists $n_{\epsilon} \in \mathbb{N}$ such that

$$
\boldsymbol{P}\left(t_{\frac{\rho_{1}^{\prime}+\rho_{1}}{2}}>n_{\epsilon}\right) \leq \frac{\epsilon}{2} .
$$

Setting $B_{1}:=\left\{\frac{t_{\frac{\rho_{1}^{\prime}+\rho_{1}}{2}}}{}>n_{\epsilon}\right\}$ and using (5.2), it follows that

$$
\epsilon \leq \boldsymbol{P}\left(A_{1}\right) \leq \epsilon / 2+\boldsymbol{P}\left(A_{1} \cap B_{1}^{c}\right)
$$

Now, we show that $\boldsymbol{P}\left(A_{1} \cap B_{1}^{c}\right)=0$. Setting

$$
C_{1}=\left\{\underline{\lim }_{n} Z_{n}<\frac{\rho_{1}^{\prime}+\rho_{1}}{2}\right\},
$$

we decompose $\boldsymbol{P}\left(A_{1} \cap B_{1}^{c}\right)$ as follows:

$$
\boldsymbol{P}\left(A_{1} \cap B_{1}^{c}\right) \leq \boldsymbol{P}\left(E_{1}\right)+\boldsymbol{P}\left(E_{2}\right)
$$

where $E_{1}=A_{1} \cap B_{1}^{c} \cap C_{1}$ and $E_{2}=A_{1} \cap B_{1}^{c} \cap C_{1}^{c}$.

Consider the term $\boldsymbol{P}\left(E_{2}\right)$. Note that on the set $C_{1}^{c}$, we have $\left\{\underline{\lim }_{n} Z_{n} \geq \frac{\rho_{1}^{\prime}+\rho_{1}}{2}\right\}$ and on the set $B_{1}^{c}$ we have $\left\{\hat{\rho}_{1, n} \leq \frac{\rho_{1}^{\prime}+\rho_{1}}{2}\right\}$ for any $n \geq n_{\epsilon}$. Hence, since $\left(B_{1}^{c} \cap C_{1}^{c}\right) \supset E_{2}$, on the set $E_{2}$ we have that $W_{1, n}=\mathbb{1}_{\left\{Z_{n} \leq \hat{\rho}_{1, n}\right\}} \stackrel{\text { a.s. }}{\rightarrow} 0$. Then, letting $\tau_{W}:=\sup \left\{k \geq 1: W_{1, k}=1\right\}$ we have $\boldsymbol{P}\left(E_{2} \cap\left\{\tau_{W}<\right.\right.$ $\infty\})=\boldsymbol{P}\left(E_{2}\right)$ and, on the set $E_{2}$, for any $n \geq \tau_{W}$ the ARRU model can be written as follows:

$$
\left\{\begin{array}{l}
Y_{1, n+1}=Y_{1, \tau_{W}}, \\
Y_{2, n+1}=Y_{2, \tau_{W}}+\sum_{i=\tau_{W}}^{n+1}\left(1-X_{i}\right) D_{2, i},
\end{array}\right.
$$

where $W_{1, i-1}=0$ for any $i \geq \tau_{W}$, and $W_{2, i-1}=1$ because $W_{2, i-1}+W_{2, i-1} \geq 1$ by construction. Now, consider an RRU model $\left\{Z_{i}^{R} ; i \geq 1\right\}$ with initial composition $\left(Y_{1,0}^{R}, Y_{2,0}^{R}\right)=\left(Y_{1, \tau_{W}}, Y_{2, \tau_{W}}\right)$ 
a.s.; the reinforcements are defined as $D_{1, i}^{R}=0$ and $D_{2, i}^{R}=D_{2, \tau_{W}+i}$ for any $i \geq 1$ a.s.; the drawing process is modeled by $X_{i+1}^{R}:=\mathbb{1}_{\left\{U_{i+1}^{R}<Z_{i}^{R}\right\}}$ and $U_{i}^{R}=U_{\tau_{W}+i}$ a.s., where $\left\{U_{n} ; n \geq 1\right\}$ is the sequence such that $X_{n+1}=\mathbb{1}_{\left\{U_{n}<Z_{n}\right\}}$ for any $n \geq 1$. Formally, this RRU model can be described for any $n \geq 1$ as follows:

$$
\left\{\begin{array}{l}
Y_{1, n+1}^{R}=Y_{1,0}^{R}=Y_{1, \tau_{W}}, \\
Y_{2, n+1}^{R}=Y_{2,0}^{R}+\sum_{i=0}^{n+1}\left(1-X_{i}^{R}\right) D_{2, i}^{R}=Y_{2, \tau_{W}}+\sum_{i=\tau_{W}}^{n+\tau_{W}+1}\left(1-X_{i}\right) D_{2, i} .
\end{array}\right.
$$

Hence, on the set $E_{2}$ we have that for any $n \geq \tau_{W}$

$$
\left(Y_{1, n}, Y_{2, n}\right)=\left(Y_{1, n-\tau_{W}}^{R}, Y_{2, n-\tau_{W}}^{R}\right)
$$

Since from Muliere, Paganoni and Secchi [22], Theorem $8, \boldsymbol{P}\left(\overline{\lim }_{n} Z_{n}^{R}=0\right)=1$, on the set $E_{2}$ we have that $\left\{\overline{\lim }_{n} Z_{n}=0\right\}$. This is incompatible with the set $A_{1}$ which includes $E_{2}$. Hence $\boldsymbol{P}\left(E_{2}\right)=0$.

We now turn to the proof that $\boldsymbol{P}\left(E_{1}\right)=0$. To this end, let

$$
\tau_{\epsilon}:=\inf \left\{k \geq n_{\epsilon}:\left\{Z_{k}<\frac{\rho_{1}^{\prime}+\rho_{1}}{2}\right\} \cap\left\{Y_{k}>\frac{b}{\left(\rho_{1}^{\prime}-\rho_{1}\right) / 2}\right\}\right\}
$$

and note that, since by result (c) of Lemma 3.1 $Y_{n} \stackrel{\text { a.s. }}{\rightarrow} \infty, \boldsymbol{P}\left(C_{1} \cap\left\{\tau_{\epsilon}<\infty\right\}\right)=\boldsymbol{P}\left(C_{1}\right)$. Moreover, on the set $B_{1}^{c}$ we have that $\left\{\hat{\rho}_{1, n} \leq \frac{\rho_{1}^{\prime}+\rho_{1}}{2}\right\}$ for any $n \geq n_{\epsilon}$. We now show by induction that on the set $B_{1}^{c} \cap C_{1}$ we have $\left\{Z_{n}<\rho_{1}^{\prime} \forall n \geq \tau_{\epsilon}\right\}$. By definition we have $Z_{\tau_{\epsilon}}<\frac{\rho_{1}^{\prime}+\rho_{1}}{2}$, and by Lemma 3.5 this implies $Z_{\tau_{\epsilon}+1}<\rho_{1}^{\prime}$; now, consider an arbitrary $n>\tau_{\epsilon}$; if $Z_{n}<\frac{\rho_{1}^{\prime}+\rho_{1}}{2}$, then by Lemma 3.5 we have $Z_{n+1}<\rho_{1}^{\prime}$; if $\frac{\rho_{1}^{\prime}+\rho_{1}}{2}<Z_{n}<\rho_{1}^{\prime}$ we have $W_{1, n}=0$ and so $Z_{n+1} \leq Z_{n}<\rho_{1}^{\prime}$. Hence, since $\left(B_{1}^{c} \cap C_{1}\right) \subset E_{1}$, on the set $E_{1}$ we have $\left\{Z_{n}<\rho_{1}^{\prime} \forall n \geq \tau_{\epsilon}\right\}$. This is incompatible with the set $A_{1}$ which also includes $E_{1}$. Hence $\boldsymbol{P}\left(E_{1}\right)=0$. Combining all together we have $\epsilon \leq \epsilon / 2+\boldsymbol{P}\left(E_{1}\right)+$ $\boldsymbol{P}\left(E_{2}\right)=\epsilon / 2$, which is impossible. Thus, we conclude that $\boldsymbol{P}\left(A_{1}^{c}\right)=\boldsymbol{P}\left(\overline{\lim }_{n} Z_{n} \leq \rho_{1}\right)=1$.

For part (b), wlog we assume $m_{1}>m_{2}$ to show that $\boldsymbol{P}\left(\overline{\lim }_{n} Z_{n} \geq \rho_{1}\right)=1$, since the proof of $\boldsymbol{P}\left(\underline{\lim }_{n} Z_{n} \leq \rho_{2}\right)=1$ when $m_{1}<m_{2}$ follows the same arguments. To this end, we now show that there cannot exist $\epsilon>0$ and $\rho^{\prime}<\rho_{1}$ such that

$$
\boldsymbol{P}\left(\overline{\lim }_{n} Z_{n}<\rho_{1}^{\prime}\right) \geq \epsilon>0 .
$$

We prove this by contradiction, using a comparison argument with an RRU model. Now suppose (5.3) holds and let $A_{2}:=\left\{\overline{\lim }_{n} Z_{n}<\rho_{1}^{\prime}\right\}$. Let

$$
R_{2}:=\left\{k \geq 0: \hat{\rho}_{1, k}<\frac{\rho_{1}^{\prime}+\rho_{1}}{2}\right\}
$$


and define the last time the process $\left\{\hat{\rho}_{1, n} ; n \geq 1\right\}$ is less than $\left(\rho_{1}^{\prime}+\rho_{1}\right) / 2$ by

$$
\tau_{\frac{\rho_{1}^{\prime}+\rho_{1}}{2}}= \begin{cases}\sup \left\{R_{2}\right\} & \text { if } R_{2} \neq \varnothing ; \\ 0 & \text { otherwise. }\end{cases}
$$

Since $\hat{\rho}_{1, n} \stackrel{\text { a.s. }}{\rightarrow} \rho_{1}$, then we have that $\boldsymbol{P}\left(\frac{\tau_{\rho_{1}^{\prime}+\rho_{1}}}{2}<\infty\right)=1$. Hence, there exists $n_{\epsilon} \in \mathbb{N}$ such that

$$
\boldsymbol{P}\left(\tau_{\frac{\rho_{1}^{\prime}+\rho_{1}}{2}}>n_{\epsilon}\right) \leq \frac{\epsilon}{2} .
$$

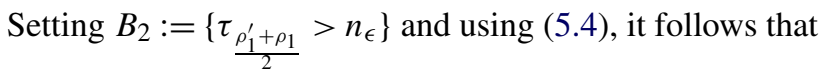

$$
\epsilon \leq \boldsymbol{P}\left(A_{2}\right) \leq \epsilon / 2+\boldsymbol{P}\left(A_{2} \cap B_{2}^{c}\right)
$$

Let $E_{3}:=A_{2} \cap B_{2}^{c}$. We now show that $\boldsymbol{P}\left(E_{3}\right)=0$. On the set $A_{2}$, we have $\left\{\overline{\lim }_{n} Z_{n} \leq \rho_{1}^{\prime}\right\}$ and on the set $B_{2}^{c}$, we have $\left\{\hat{\rho}_{1, n} \geq \frac{\rho_{1}^{\prime}+\rho_{1}}{2}\right\}$ for any $n \geq n_{\epsilon}$. Hence, on the set $E_{3}$ we have that $W_{1, n}=\mathbb{1}_{\left\{Z_{n} \leq \hat{\rho}_{1, n}\right\}} \stackrel{\text { a.s. }}{\rightarrow}$ 1. Then, letting $\tau_{W}:=\sup \left\{k \geq 1: W_{1, n}=0\right\}$ we have $\boldsymbol{P}\left(E_{3} \cap\left\{\tau_{W}<\right.\right.$ $\infty\})=\boldsymbol{P}\left(E_{3}\right)$. Now, analogously to the proof of $\boldsymbol{P}\left(E_{2}\right)=0$, we can use comparison arguments with an RRU model to show that on the set $E_{3}$ we have $\left\{\overline{\lim }_{n} Z_{n}=1\right\}$. This is incompatible with the set $A_{2}$, which also includes $E_{3}$. Hence $\boldsymbol{P}\left(E_{3}\right)=0$. Combining all together we have $\epsilon \leq \epsilon / 2+\boldsymbol{P}\left(E_{3}\right)=\epsilon / 2$, which is impossible. Thus, we conclude that the event $A_{2}^{c}=\left\{\varlimsup_{\lim _{n}} Z_{n} \geq \rho_{1}\right\}$ occurs with probability one.

For part (c), note that, combining (a) and (b), we have shown that

$$
\begin{cases}\boldsymbol{P}\left(\overline{\lim }_{n} Z_{n}=\rho_{1}\right)=1 & \text { if } m_{1}>m_{2}, \\ \boldsymbol{P}\left(\rho_{2} \leq \underline{\lim }_{n} Z_{n} \leq \varlimsup_{\overline{\lim }} Z_{n} \leq \rho_{1}\right)=1 & \text { if } m_{1}=m_{2}, \\ \boldsymbol{P}\left(\underline{\lim }_{n} Z_{n}=\rho_{2}\right)=1 & \text { if } m_{1}<m_{2} .\end{cases}
$$

Therefore, if the process $\left\{Z_{n} ; n \geq 1\right\}$ converges almost surely, we obtain (2.6). Wlog, assume $m_{1} \geq m_{2}$, since the proof of the case $m_{1} \leq m_{2}$ is completely analogous.

First, let $d, u, \gamma$ and $\rho_{1}^{\prime}\left(d<u<\gamma<\rho_{1}^{\prime}<\rho_{1}\right)$ be four constants in $(0,1)$. Let $\left\{\tau_{j}(d, u) ; j \geq 1\right\}$ and $\left\{t_{j}(d, u) ; j \geq 1\right\}$ be the sequences of random variables defined in (3.2). Since $d$ and $u$ are fixed in this proof, we sometimes denote $\tau_{j}(d, u)$ by $\tau_{j}$ and $t_{j}(d, u)$ by $t_{j}$. It is easy to see that $\tau_{n}$ and $t_{n}$ are stopping times with respect to $\left\{\mathcal{F}_{n} ; n \geq 1\right\}$.

Recall that, by Lemma 3.2, we have that for every $0<d<u<1$

$$
\begin{aligned}
Z_{n} \text { converges a.s. } & \Leftrightarrow \boldsymbol{P}\left(t_{n}(d, u)<\infty\right) \rightarrow 0 \\
& \Leftrightarrow \sum_{n=1}^{\infty} \boldsymbol{P}\left(t_{n+1}(d, u)=\infty \mid t_{n}(d, u)<\infty\right)=\infty .
\end{aligned}
$$

Now, to prove that $Z_{n}$ converges a.s., it is sufficient to show that

$$
\boldsymbol{P}\left(t_{n}(d, u)<\infty\right) \rightarrow 0,
$$


for all $0<d<u<1$. Suppose $Z_{n}$ does not converges a.s. This implies that $\boldsymbol{P}\left(t_{n}<\infty\right) \downarrow \phi_{1}>0$, since $\boldsymbol{P}\left(t_{n}<\infty\right)$ is a non-increasing sequence. We will show that for large $j$ there exists a constant $\phi<1$ dependent on $\phi_{1}$, such that

$$
\boldsymbol{P}\left(t_{j+1}<\infty \mid t_{j}<\infty\right) \leq \phi .
$$

This result implies that $\sum_{n} \boldsymbol{P}\left(t_{n+1}=\infty \mid t_{n}<\infty\right)=\infty$, establishing by Lemma 3.2 that $\boldsymbol{P}\left(t_{n}<\right.$ $\infty)$ converges to zero as $n$ goes to infinity, which is a contradiction.

Consider the term $\boldsymbol{P}\left(t_{i+1}<\infty \mid t_{i}<\infty\right)$. First, let us denote by $\tau_{\rho_{1}^{\prime}}$ the last time the process $\hat{\rho}_{1, n}$ is below $\rho_{1}^{\prime}$, that is,

$$
\tau_{\rho_{1}^{\prime}}= \begin{cases}\sup \left\{n \geq 1: \hat{\rho}_{1, n} \leq \rho_{1}^{\prime}\right\} & \text { if }\left\{n \geq 1: \hat{\rho}_{1, n} \leq \rho_{1}^{\prime}\right\} \neq \varnothing \\ 0 & \text { otherwise }\end{cases}
$$

Since $\hat{\rho}_{1, n} \stackrel{\text { a.s. }}{\rightarrow} \rho_{1}$, we have that $\boldsymbol{P}\left(\tau_{\rho_{1}^{\prime}}<\infty\right)=1$. Hence, for any $\epsilon \in\left(0, \frac{1}{2}\right)$ there exists $n_{\epsilon} \in \mathbb{N}$ such that

$$
\frac{1}{\phi_{1}} \boldsymbol{P}\left(\tau_{\rho_{1}^{\prime}}>n_{\epsilon}\right) \leq \epsilon .
$$

By denoting $\boldsymbol{P}_{i}(\cdot)=\boldsymbol{P}\left(\cdot \mid t_{i}<\infty\right)$ and using $t_{i} \leq \tau_{i} \leq t_{i+1}$ we obtain

$$
\boldsymbol{P}\left(t_{i+1}<\infty \mid t_{i}<\infty\right) \leq \boldsymbol{P}_{i}\left(\tau_{i}<\infty\right) .
$$

Hence,

$$
\boldsymbol{P}_{i}\left(\tau_{i}<\infty\right) \leq \boldsymbol{P}_{i}\left(\left\{\tau_{i}<\infty\right\} \cap\left\{\tau_{\rho_{1}^{\prime}} \leq n_{\epsilon}\right\}\right)+\boldsymbol{P}_{i}\left(\tau_{\rho_{1}^{\prime}}>n_{\epsilon}\right)
$$

We start with the second term in (5.7). Note that

$$
\boldsymbol{P}_{i}\left(\tau_{\rho_{1}^{\prime}}>n_{\epsilon}\right) \leq \frac{\boldsymbol{P}\left(\tau_{\rho_{1}^{\prime}}>n_{\epsilon}\right)}{\boldsymbol{P}\left(t_{i}<\infty\right)} \leq \frac{\boldsymbol{P}\left(\tau_{\rho_{1}^{\prime}}>n_{\epsilon}\right)}{\phi_{1}} \leq \epsilon,
$$

where the last inequality follows from (5.6).

Now, consider the first term in (5.7). Since the probability is conditioned on the set $\left\{t_{i}<\infty\right\}$, in what follows we will consider the urn process at times $n$ after the stopping time $t_{i}$. Since we want to show (5.5) for large $i$, we can choose an integer $i \geq n_{\epsilon}$ and

$$
i>\log _{\frac{u(1-d)}{d(1-u)}}\left(\frac{b}{Y_{0}(\gamma-u)}\right) \text {, }
$$

so that

(i) $t_{i} \geq i \geq n_{\epsilon}$ a.s.;

(ii) from Lemma 3.3, we have that $Y_{\tau_{i}}>b /(\gamma-u)$ a.s.

These two properties imply respectively that, on the set $\left\{n \geq t_{i}\right\}$

(i) $\hat{\rho}_{1, n} \geq \rho_{1}^{\prime}$, since from $\left\{\tau_{\rho_{1}^{\prime}} \leq n_{\epsilon}\right\}$ we have that $n \geq \tau_{\rho_{1}^{\prime}}$; 
(ii) $Z_{t_{i}} \in(u, \gamma)$, since $Z_{t_{i}-1} \leq u$ and $Z_{t_{i}}>u$ and from Lemma 3.5 we have that $\mid Z_{n}-$ $Z_{n-1} \mid<(\gamma-u)$.

Now, let us define two sequences of stopping times $\left\{t_{n}^{*} ; n \geq 1\right\}$ and $\left\{\tau_{n}^{*} ; n \geq 1\right\}$, where $t_{n}^{*}$ represents the first time after $\tau_{n-1}^{*}$ the process $Z_{t_{i}+n}$ up-crosses $\rho_{1}^{\prime}$, while $\tau_{n}^{*}$ represents the first time after $t_{n}^{*}$ the process $Z_{t_{i}+n}$ down-crosses $\gamma$. Formally, let $\tau_{0}^{*}=0$ and define for every $j \geq 1$ two stopping times

$$
\begin{aligned}
& t_{j}^{*}= \begin{cases}\inf \left\{n>\tau_{j-1}^{*}: Z_{t_{i}+n}>\rho_{1}^{\prime}\right\} & \text { if }\left\{n>\tau_{j}^{*}: Z_{t_{i}+n}>\rho_{1}^{\prime}\right\} \neq \varnothing ; \\
+\infty & \text { otherwise, }\end{cases} \\
& \tau_{j}^{*}= \begin{cases}\inf \left\{n>t_{j}^{*}: Z_{t_{i}+n} \leq \gamma\right\} & \text { if }\left\{n>t_{j-1}^{*}: Z_{t_{i}+n} \leq \gamma\right\} \neq \varnothing ; \\
+\infty & \text { otherwise. }\end{cases}
\end{aligned}
$$

Note that, since $Z_{t_{i}+\tau_{j}^{*}-1} \geq \gamma$ and $Z_{t_{i}+\tau_{j}^{*}}<\gamma$, from (ii) we have that $Z_{t_{i}+\tau_{j}^{*}} \in(u, \gamma)$.

For any $j \geq 0$, let $\left\{\tilde{Z}_{n}^{j} ; n \geq 1\right\}$ be an RRU model defined as follows:

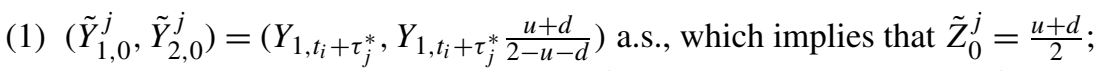

(2) the drawing process is modeled by $\tilde{X}_{n+1}^{j}=\mathbb{1}_{\left\{\tilde{U}_{n+1}^{j}<\tilde{Z}_{n}^{j}\right\}}$, where $\tilde{U}_{n+1}^{j}=U_{t_{i}+\tau_{j}^{*}+n+1}$ a.s. and $U_{n}$ is such that $X_{n}=\mathbb{1}_{\left\{U_{n}<Z_{n-1}\right\}}$;

(3) the reinforcements are defined as $\tilde{D}_{2, n+1}^{j}=D_{2, t_{i}+\tau_{j}^{*}+n+1}+\left(m_{1}-m_{2}\right), \tilde{D}_{1, n+1}^{j}=$ $D_{1, t_{i}+\tau_{j}^{*}+n+1}$ a.s.; this means $\boldsymbol{E}\left[\tilde{D}_{1, n}^{j}\right]=\boldsymbol{E}\left[\tilde{D}_{2, n}^{j}\right]$ for any $n \geq 1$;

(4) the urn process evolves as an RRU model, that is, for any $n \geq 0$

$$
\left\{\begin{array}{l}
\tilde{Y}_{1, n+1}^{j}=\tilde{Y}_{1, n}^{j}+\tilde{X}_{n+1}^{j} \tilde{D}_{1, n+1}^{j}, \\
\tilde{Y}_{2, n+1}^{j}=\tilde{Y}_{2, n}^{j}+\left(1-\tilde{X}_{n+1}^{j}\right) \tilde{D}_{2, n+1}^{j}, \\
\tilde{Y}_{n+1}^{j}=\tilde{Y}_{1, n+1}^{j}+\tilde{Y}_{2, n+1}^{j}, \\
\tilde{Z}_{n+1}^{j}=\frac{\tilde{Y}_{1, n+1}^{j}}{\tilde{Y}_{n+1}^{j}} .
\end{array}\right.
$$

We will compare the process $\left\{\tilde{Z}_{n}^{j} ; n \geq 1\right\}$ with the ARRU process $\left\{Z_{t_{i}+n} ; n \geq 1\right\}$. Note that at time $n$, we have defined only the processes $\tilde{Z}^{j}$ such that $\tau_{j}^{*}<n$.

We will prove, by induction, that on the set $\left\{\tau_{\rho_{1}^{\prime}} \leq n_{\epsilon}\right\}$, for any $j \in \mathbb{N}$ and for any $n \leq t_{j+1}^{*}-\tau_{j}^{*}$

$$
\tilde{Z}_{n}^{j}<Z_{t_{i}+\tau_{j}^{*}+n}, \quad \tilde{Y}_{2, n}^{j} \geq Y_{2, t_{i}+\tau_{j}^{*}+n}, \quad \tilde{Y}_{1, n}^{j}<Y_{1, t_{i}+\tau_{j}^{*}+n} .
$$

In other words, we will show, provided that $t_{i}>\tau_{\rho_{1}^{\prime}}$, that for each $j \geq 1$ the process $\tilde{Z}_{n}^{j}$ is always dominated by the original process $Z_{t_{i}+\tau_{j}^{*}+n}$, as long as $Z_{t_{i}+\tau_{j}^{*}+n}$ is dominated by $\rho_{1}^{\prime}$ (i.e., for $n \leq t_{j+1}^{*}-\tau_{j}^{*}$ ). By construction, we have that

$$
\tilde{Z}_{0}^{j}=\frac{d+u}{2}<u<Z_{t_{i}+\tau_{j}^{*}}, \quad \tilde{Y}_{1,0}^{j}=Y_{1, t_{i}+\tau_{j}^{*}}
$$


which immediately implies $\tilde{Y}_{2,0}^{j}>Y_{2, t_{i}+\tau_{j}^{*}}$. To this end, we assume (5.8) by induction hypothesis. First, we will show that $\tilde{Y}_{2, n+1}^{j}>Y_{2, t_{i}+\tau_{j}^{*}+n+1}$. Since from (5.8) $\tilde{Z}_{n}^{j}<Z_{t_{i}+\tau_{j}^{*}+n}$ for $n \leq t_{j+1}^{*}-$ $\tau_{j}^{*}$, by construction we obtain that

$$
\tilde{X}_{n+1}^{j}=\mathbb{1}_{\left\{\tilde{U}_{n}^{j}<\tilde{Z}_{n}^{j}\right\}} \leq \mathbb{1}_{\left\{U_{t_{i}+\tau_{j}^{*}+n+1}<Z_{t_{i}+\tau_{j}^{*}+n}\right\}}=X_{t_{i}+\tau_{j}^{*}+n+1} .
$$

As a consequence, since $W_{n} \leq 1$ for any $n \geq 1$, we have that

$$
\begin{aligned}
\left(Y_{2, t_{i}+\tau_{j}^{*}+n+1}-Y_{2, t_{i}+\tau_{j}^{*}+n}\right) & =\left(1-X_{t_{i}+\tau_{j}^{*}+n+1}\right) D_{2, t_{i}+\tau_{j}^{*}+n+1} W_{2, t_{i}+\tau_{j}^{*}+n} \\
& \leq\left(1-\tilde{X}_{n+1}^{j}\right) \tilde{D}_{2, n+1}^{j} \\
& =\left(\tilde{Y}_{2, n+1}^{j}-\tilde{Y}_{2, n}^{j}\right)
\end{aligned}
$$

which, using hypothesis (5.8), implies $\tilde{Y}_{2, n+1}^{j}>Y_{2, t_{i}+\tau_{j}^{*}+n+1}$. Similarly, we now show that $\tilde{Y}_{1, n+1}^{j} \leq Y_{1, t_{i}+\tau_{j}^{*}+n+1}$. We have

$$
\left(Y_{1, t_{i}+\tau_{j}^{*}+n+1}-Y_{1, t_{i}+\tau_{j}^{*}+n}\right)=X_{t_{i}+\tau_{j}^{*}+n+1} D_{1, t_{i}+\tau_{j}^{*}+n+1} W_{1, t_{i}+\tau_{j}^{*}+n}
$$

From (i) we have that, as long as $Z$ remains below $\rho_{1}^{\prime}, Z$ is also above the process $\hat{\rho}_{1, n}$. Since we consider the behavior of $Z_{t_{i}+\tau_{j}^{*}+n}$ when it is below $\rho_{1}^{\prime}$, that is, $n \leq \tau_{j+1}^{*}-t_{j}^{*}$, we have that $W_{1, t_{i}+\tau_{j}^{*}+n}=1$. Thus,

$$
\left(Y_{1, t_{i}+\tau_{j}^{*}+n+1}-Y_{1, t_{i}+\tau_{j}^{*}+n}\right) \geq \tilde{X}_{n+1}^{j} \tilde{D}_{1, n+1}^{j}=\left(\tilde{Y}_{1, n+1}^{j}-\tilde{Y}_{1, n}^{j}\right)
$$

which using hypothesis (5.8) implies $\tilde{Y}_{1, n+1}^{j} \leq Y_{1, t_{i}+\tau_{j}^{*}+n+1}$. Thus, we have shown that, on the set $\left\{\tau_{\rho_{1}^{\prime}} \leq n_{\epsilon}\right\}$, for any $n \leq t_{j+1}^{*}-\tau_{j}^{*}, \tilde{Z}_{n+1}^{j}<Z_{t_{i}+\tau_{j}^{*}+n+1}, \tilde{Y}_{1, n+1}^{j} \leq Y_{1, t_{i}+\tau_{j}^{*}+n+1}$ and $\tilde{Y}_{2, n+1}^{j}>$ $Y_{2, t_{i}+\tau_{j}^{*}+n+1}$ hold.

Now, for any $j \geq 1$, let $T_{j}$ be the stopping time for $\tilde{Z}_{n}^{j}$ to exit from $(d, u)$, that is,

$$
T_{j}= \begin{cases}\inf \left\{R_{3}\right\} & \text { if } R_{3} \neq \varnothing \\ +\infty & \text { otherwise }\end{cases}
$$

where $R_{3}:=\left\{n \geq 1: \tilde{Z}_{n}^{j} \leq d\right.$ or $\left.\tilde{Z}_{n}^{j} \geq u\right\}$. Note that, on the set $\left\{\tau_{\rho_{1}^{\prime}} \leq n_{\epsilon}\right\}$,

$$
\begin{aligned}
\left\{\tau_{i}<\infty\right\} & =\left\{\inf _{n \geq 1}\left\{Z_{t_{i}+n}\right\}<d\right\} \subset\left(\bigcup_{j: \tau_{j}^{*} \leq n}\left\{\inf _{n \geq 1}\left\{\tilde{Z}_{n-\tau_{j}^{*}}^{j}\right\}<d\right\}\right) \\
& \subset\left(\bigcup_{j=0}^{\infty}\left\{T_{j}<\infty\right\}\right) .
\end{aligned}
$$


Hence, by denoting $\boldsymbol{P}_{i}(\cdot)=\boldsymbol{P}\left(\cdot \mid t_{i}<\infty\right)$ and $\boldsymbol{E}_{i}[\cdot]=\boldsymbol{E}\left[\cdot \mid t_{i}<\infty\right]$, we have that

$$
\begin{aligned}
\boldsymbol{P}_{i}\left(\left\{\tau_{i}<\infty\right\} \cap\left\{\tau_{\rho_{1}^{\prime}} \leq n_{\epsilon}\right\}\right) & \leq \boldsymbol{P}_{i}\left(\left\{\bigcup_{j=0}^{\infty}\left\{T_{j}<\infty\right\}\right\} \cap\left\{\tau_{\rho_{1}^{\prime}} \leq n_{\epsilon}\right\}\right) \\
& \leq \sum_{j=0}^{\infty} \boldsymbol{P}_{i}\left(\left\{T_{j}<\infty\right\} \cap\left\{\tau_{\rho_{1}^{\prime}} \leq n_{\epsilon}\right\}\right),
\end{aligned}
$$

and, by setting $h=\frac{u-d}{2}$, each term of the series is less or equal than

$$
\boldsymbol{P}_{i}\left(\left\{\sup _{n \geq 1}\left|\tilde{Z}_{n}^{j}-\tilde{Z}_{0}^{j}\right| \geq h\right\} \cap\left\{\tau_{\rho_{1}^{\prime}} \leq n_{\epsilon}\right\}\right) \leq \boldsymbol{P}_{i}\left(\sup _{n \geq 1}\left|\tilde{Z}_{n}^{j}-\tilde{Z}_{0}^{j}\right| \geq h\right) .
$$

Note that $\left\{\tilde{Z}_{n}^{j} ; n \geq 1\right\}$ is the proportion of red balls in an RRU model with same reinforcement means. Then, by using Lemma 3.4 we obtain

$$
\begin{aligned}
\boldsymbol{P}_{i}\left(\sup _{n \geq 1}\left|\tilde{Z}_{n}^{j}-\tilde{Z}_{0}^{j}\right| \geq h\right) & =\boldsymbol{E}_{i}\left[\boldsymbol{P}\left(\sup _{n \geq 1}\left|\tilde{Z}_{n}^{j}-\tilde{Z}_{0}^{j}\right| \geq h \mid \mathcal{F}_{\tau_{i}+t_{j}^{*}}\right)\right] \\
& \leq \boldsymbol{E}_{i}\left[\frac{b}{Y_{t_{j}^{*}}}\right]\left(\frac{4}{h^{2}}+\frac{2}{h}\right)
\end{aligned}
$$

Moreover, by using Lemma 3.3, the right-hand side can be expressed as

$$
\boldsymbol{E}_{i}\left[\frac{b}{Y_{t_{i}}}\right]\left(\frac{\rho_{1}^{\prime}(1-\gamma)}{\gamma\left(1-\rho_{1}^{\prime}\right)}\right)^{j}\left(\frac{4}{h^{2}}+\frac{2}{h}\right) .
$$

Since by result (c) of Lemma $3.1 Y_{n}$ converges a.s. to infinity, and since $\tau_{i} \rightarrow \infty$ a.s. because $\tau_{i} \geq i$, we have that $\boldsymbol{E}_{i}\left[Y_{t_{i}}^{-1}\right]$ tends to zero as $i$ increases. As a consequence, we can choose an integer $i$ large enough such that

$$
\boldsymbol{E}_{i}\left[\frac{b}{Y_{t_{i}}}\right]\left(\frac{4}{h^{2}}+\frac{2}{h}\right)\left(\frac{1-\rho_{1}^{\prime}}{1-\rho_{1}^{\prime} / \gamma}\right)<\frac{1}{2},
$$

which by setting $\phi=1 / 2+\epsilon$ implies (5.5), i.e.

$$
\boldsymbol{P}\left(t_{i+1}<\infty \mid t_{i}<\infty\right) \leq \phi<1 .
$$

This concludes the proof.

Proof of Lemma 2.6. We divide the proof in two parts:

(i) $m_{1} \neq m_{2}$ and $0<\rho_{2}<\rho_{1}<1$;

(ii) $m_{1}=m_{2}$ and $0 \leq \rho_{2}<\rho_{1} \leq 1$, on the set $\left\{Z_{\infty} \neq\{0,1\}\right\}$. 
For part (i), assume $m_{1}>m_{2}$, since the proof in the case $m_{1}<m_{2}$ follows the same arguments. In this case $\min \left\{m_{1}, m_{2}\right\}=m_{2}$ and, by using Theorem 2.4 , we have $Z_{n} \stackrel{\text { a.s. }}{\rightarrow} \rho_{1}$; thus, since $\hat{\rho}_{2, n} \stackrel{\text { a.s. }}{\rightarrow} \rho_{2}$ and $\rho_{1}>\rho_{2}$, denoting by $\tau \in \mathbb{N}$ the last time $Z_{n}$ crosses $\hat{\rho}_{2, n}$, that is, $\tau:=\sup \{k \geq$ $\left.1, Z_{k}<\hat{\rho}_{2, k}\right\}$, we have that $\boldsymbol{P}(\tau<\infty)=1$. Then, since $\{\tau \leq n\} \subset\left\{W_{2, k}=1, \forall k \geq n\right\}$, we use the following decomposition, on the set $\{\tau \leq n\}$,

$$
\frac{Y_{2, n}}{n}=\frac{1}{n} \sum_{i=1}^{n}\left(1-X_{i}\right) D_{2, i} W_{2, i-1}=\mathcal{W}_{0, n}+\mathcal{W}_{1, n},
$$

where

$$
\begin{aligned}
& \mathcal{W}_{0, n}:=\frac{1}{n} \sum_{i=1}^{\tau}\left(1-X_{i}\right) D_{2, i}\left(W_{2, i-1}-1\right), \\
& \mathcal{W}_{1, n}:=\frac{1}{n} \sum_{i=\tau}^{n}\left(1-X_{i}\right) D_{2, i} .
\end{aligned}
$$

Since $\boldsymbol{P}(\tau<\infty)=1$, we have $\mathcal{W}_{0, n} \stackrel{\text { a.s. }}{\rightarrow} 0$, while since

$$
\boldsymbol{E}\left[\left(1-X_{i}\right) D_{2, i} \mid \mathcal{F}_{i-1}\right]=\left(1-Z_{i-1}\right) m_{2} \stackrel{\text { a.s. }}{\rightarrow}\left(1-Z_{\infty}\right) m_{2},
$$

we have that $\mathcal{W}_{1, n} \stackrel{\text { a.s. }}{\rightarrow}\left(1-Z_{\infty}\right) m_{2}$. Finally, since $Y_{n}=\left(1-Z_{n}\right)^{-1} Y_{2, n}$, we have $\frac{Y_{n}}{n} \stackrel{\text { a.s. }}{\rightarrow} m_{2}=$ $\min \left\{m_{1}, m_{2}\right\}$.

For part (ii), since $m_{1}=m_{2}=m$, by using Theorem 2.4 we have $Z_{n} \stackrel{\text { a.s. }}{\rightarrow} Z_{\infty} \in\left[\rho_{2}, \rho_{1}\right]$; then, on the set $\left\{Z_{\infty} \in(0,1)\right\}$, we can follow the arguments of part (i), so obtaining

$$
\frac{Y_{2, n}}{n} \stackrel{\text { a.s. }}{\rightarrow}\left(1-Z_{\infty}\right) m, \quad \frac{Y_{1, n}}{n} \stackrel{\text { a.s. }}{\rightarrow} Z_{\infty} m .
$$

Thus, $\frac{Y_{n}}{n}=\frac{Y_{1, n}}{n}+\frac{Y_{2, n}}{n} \stackrel{\text { a.s. }}{\rightarrow} m$.

The proof of Lemma 2.7 is based on comparison arguments between the ARRU and RRU model. Specifically, for any $n_{0} \geq 1$, we consider an RRU process $\left\{\tilde{Z}_{k}\left(n_{0}\right) ; k \geq 0\right\}$ coupled with the ARRU process $\left\{Z_{n_{0}+k} ; k \geq 0\right\}$ as follows: the initial composition is $\left(\tilde{Y}_{1,0}\left(n_{0}\right), \tilde{Y}_{2,0}\left(n_{0}\right)\right)=$ $\left(Y_{1, n_{0}}, Y_{2, n_{0}}\right)$ and for any $k \geq 1$

$$
\left\{\begin{array}{l}
\tilde{Y}_{1, k}\left(n_{0}\right)=\tilde{Y}_{1, k-1}\left(n_{0}\right)+\tilde{X}_{1, k}\left(n_{0}\right) D_{1, k}, \\
\tilde{Y}_{2, k}\left(n_{0}\right)=\tilde{Y}_{2, k-1}\left(n_{0}\right)+\left(1-\tilde{X}_{k}\left(n_{0}\right)\right) D_{2, k},
\end{array}\right.
$$

where $\tilde{X}_{k}\left(n_{0}\right)=\mathbb{1}_{\left\{U_{k} \leq \tilde{Z}_{k-1}\left(n_{0}\right)\right\}}$. The relation between $\tilde{Z}_{k}\left(n_{0}\right)$ and $Z_{n_{0}+k}$ required in the proof of Lemma 2.7 is expressed in the following result. 
Lemma 5.1. For any $n_{0}, n_{1} \geq 1$, we have that

$$
\left(\bigcap_{k=1}^{n_{1}}\left\{\hat{\rho}_{2, n_{0}+k} \leq Z_{n_{0}+k} \leq \hat{\rho}_{1, n_{0}+k}\right\}\right) \subset\left(\bigcap_{k=1}^{n_{1}}\left\{Z_{n_{0}+k}=\tilde{Z}_{k}\left(n_{0}\right)\right\}\right) .
$$

Proof. First, consider the dynamics of the RRU process $\left\{\tilde{Z}_{k}\left(n_{0}\right) ; k \geq 0\right\}$ expressed in (5.9) and the dynamics of the ARRU process $\left\{Z_{n_{0}+k} ; k \geq 0\right\}$ expressed as follows:

$$
\left\{\begin{array}{l}
Y_{1, n_{0}+k}=Y_{1, n_{0}+k-1}+X_{1, n_{0}+k} D_{1, n_{0}+k} W_{1, n_{0}+k-1}, \\
Y_{2, n_{0}+k}=Y_{2, n_{0}+k-1}+\left(1-X_{1, n_{0}+k}\right) D_{2, n_{0}+k} W_{2, n_{0}+k-1},
\end{array}\right.
$$

where $X_{n_{0}+k}=\mathbb{1}_{\left\{U_{k} \leq Z_{n_{0}+k-1}\right\}}$. Hence, (5.10) follows by noticing that for any $1 \leq k \leq n_{1}$

$$
\left\{\hat{\rho}_{2, n_{0}+k} \leq Z_{n_{0}+k} \leq \hat{\rho}_{1, n_{0}+k}\right\} \subset\left\{W_{1, n_{0}+k-1}=W_{2, n_{0}+k-1}=1\right\}
$$

Proof of Lemma 2.7. The proof is structured as follows: we assume there exist $x \in\left(\rho_{2}, \rho_{1}\right)$ and $p>0$ such that $\boldsymbol{P}\left(Z_{\infty}=x\right)=p$ and we show that this assumption leads to a contradiction. To this end, fix $\epsilon>0$ such that $\rho_{2}<x-\epsilon<x+\epsilon<\rho_{1}$ and denote by $\tau \in \mathbb{N}$ the last time $Z_{n}$ exceeds $I_{\epsilon}:=(x-\epsilon, x+\epsilon)$ : formally,

$$
\tau= \begin{cases}\sup \left\{k>1: Z_{k} \notin I_{\epsilon},\right\} & \text { if }\left\{k>1: Z_{k} \notin I_{\epsilon}\right\} \neq \varnothing ; \\ -\infty & \text { otherwise. }\end{cases}
$$

Since $\left\{Z_{\infty}=x\right\} \subset\{\tau<\infty\}$ and by (2.5) $\hat{\rho}_{j, n} \stackrel{\text { a.s. }}{\rightarrow} \rho_{j} \notin I_{\epsilon}, j \in\{1,2\}$, there exists an integer $k_{0} \in \mathbb{N}$ such that,

$$
\boldsymbol{P}\left(\left\{\hat{\rho}_{j, n} \notin I_{\epsilon}, \forall n \geq k_{0}\right\} \cap\left\{\tau \leq k_{0}\right\} \cap\left\{Z_{\infty}=x\right\}\right) \geq \frac{p}{2} .
$$

Now, by using Lemma 5.1, we have that

$$
\left(\left\{\hat{\rho}_{j, n} \notin I_{\epsilon}, \forall n \geq k_{0}\right\} \cap\left\{\tau \leq k_{0}\right\}\right) \subset\left\{Z_{k_{0}+n}=\tilde{Z}_{n}\left(k_{0}\right), \forall n \geq k_{0}\right\},
$$

and hence (5.12) is equivalent to

$$
\boldsymbol{P}\left(\left\{\hat{\rho}_{j, n} \notin I_{\epsilon}, \forall n \geq k_{0}\right\} \cap\left\{\tau \leq k_{0}\right\} \cap\left\{\tilde{Z}_{\infty}\left(k_{0}\right)=x\right\}\right) \geq \frac{p}{2} .
$$

Finally, the contradiction follows by noticing that by Aletti, May and Secchi [3], Theorem 2, for an RRU model, we have $\boldsymbol{P}\left(\tilde{Z}_{\infty}\left(k_{0}\right)=x\right)=0$.

Remark 5.2. As described in Remark 4.10, the boundedness of $u(\cdot)$ plays a critical role in the proofs. Additionally, when $m_{1}=m_{2}$ the weak law of large numbers (even for a bounded $u(\cdot)$ ) is unclear. Here, the behavior of the thresholds $\hat{\rho}_{1, n}$ and $\hat{\rho}_{2, n}$ is much more erratic and linking this behavior with the tail conditions of $u\left(\xi_{1,1}\right)$ and $u\left(\xi_{2,1}\right)$ remains a challenge. 


\section{Proofs of limit distribution of the proportion of sampled ball colors}

We start by presenting the limit distribution of the proportion of sampled ball colors for the RRU model.

Proof of Theorem 2.8. Note that

$$
\sqrt{n}\left(\frac{N_{1 n}}{n}-Z_{\infty}\right)=T_{1 n}+T_{2 n}
$$

where

$$
T_{1 n}:=n^{-1 / 2}\left(N_{1 n}-\sum_{i=1}^{n} Z_{i-1}\right), \quad T_{2 n}:=n^{-1 / 2} \sum_{i=1}^{n}\left(Z_{i-1}-Z_{\infty}\right) .
$$

Now, calling $\Delta Z_{j}=Z_{j}-Z_{j-1}$ and $(j \wedge n):=\min \{j, n\}$, we have that

$$
\begin{aligned}
T_{2 n} & =n^{-1 / 2} \sum_{i=1}^{n} \sum_{j=i}^{\infty}\left(-\Delta Z_{j}\right)=-n^{-1 / 2} \sum_{j=1}^{\infty} \sum_{i=1}^{j \wedge n} \Delta Z_{j} \\
& =-n^{-1 / 2} \sum_{j=1}^{\infty}(j \wedge n) \Delta Z_{j}=-\left(T_{3 n}+T_{4 n}\right),
\end{aligned}
$$

where, since $(j \wedge n)=n$ for all $j \geq n+1$, we have

$$
T_{3 n}:=n^{-1 / 2} \sum_{j=1}^{n} j \Delta Z_{j}, \quad T_{4 n}:=n^{1 / 2}\left(Z_{\infty}-Z_{n}\right)
$$

Now, by using the Doob's decomposition $\Delta Z_{j}=\Delta M_{j}+\Delta A_{j}$ (see Durrett [13]), where $\boldsymbol{E}\left[\Delta M_{j} \mid \mathcal{F}_{j-1}\right]=0$ and $A_{j} \in \mathcal{F}_{j-1}$, we have $T_{3 n}=T_{5 n}+T_{6 n}$, where

$$
T_{5 n}:=n^{-1 / 2} \sum_{j=1}^{n} j \Delta M_{j}, \quad T_{6 n}:=n^{-1 / 2} \sum_{j=1}^{n} j \Delta A_{j} .
$$

Then, recalling that

$$
\sqrt{n}\left(\frac{N_{1 n}}{n}-Z_{\infty}\right)=T_{1 n}-T_{4 n}-T_{5 n}-T_{6 n},
$$

the limit distribution is established by proving the following results:

(a) $T_{4 n} \mid \mathcal{F}_{n} \stackrel{d}{\rightarrow} \mathcal{N}\left(0, \Sigma_{a}\right)$ (stably), where $\Sigma_{a}=Z_{\infty}\left(1-Z_{\infty}\right)\left(1+\frac{\bar{\Sigma}}{m^{2}}\right)$;

(b) $T_{6 n} \stackrel{p}{\rightarrow} 0$; 
(c) $\left(T_{1 n}-T_{5 n}\right) \stackrel{d}{\rightarrow} \mathcal{N}\left(0, \Sigma_{c}\right)$ (stably), where $\Sigma_{c}=Z_{\infty}\left(1-Z_{\infty}\right) \frac{\bar{\Sigma}}{m^{2}}$;

(d) $T_{4 n}+\left(T_{1 n}-T_{5 n}\right) \stackrel{d}{\rightarrow} \mathcal{N}\left(0, \Sigma_{a}+\Sigma_{c}\right)$ (stably).

Part (a) follows from Aletti, May and Secchi [3], Theorem 1, Crimaldi, Letta and Pratelli [10], Crimaldi [9].

For part (b), by using result (a) of Lemma 3.1, for any $j \geq 0$, we have that

$$
\Delta A_{j}=\boldsymbol{E}\left[\Delta Z_{j} \mid \mathcal{F}_{j-1}\right]=Z_{j-1}\left(1-Z_{j-1}\right) B_{j-1},
$$

with $W_{1, j-1}=W_{2, j-1}=1$ (since for any $j \geq 1$, the process is an RRU model). By using Aletti, May and Secchi [3], Lemma 2, we have $\left|B_{j-1}\right|<c_{1} Y_{j-1}^{-2}$ a.s. for some constant $c_{1}>0$, and hence

$$
T_{6 n} \leq n^{-1 / 2} \sum_{j=1}^{n} j\left|\Delta A_{j}\right| \leq c_{1} n^{-1 / 2} \sum_{j=1}^{n} j Y_{j-1}^{-2}
$$

in addition, by using Aletti, May and Secchi [3], Lemma 3, we have $\boldsymbol{E}\left[Y_{j-1}^{-2}\right] \leq c_{2}(j-1)^{-2}$ for some constant $c_{2}>0$ and hence

$$
\boldsymbol{E}\left[T_{6 n}\right] \leq c_{1} c_{2} n^{-1 / 2} \sum_{j=1}^{n} j(j-1)^{-2}=O\left(n^{-1 / 2} \log (n)\right) .
$$

Thus, (b) follows.

For part (c), let $T_{1 n}-T_{5 n}=\sum_{j=1}^{n} \Delta S_{j n}$ where

$$
\Delta S_{j n}:=n^{-1 / 2}\left(X_{j}-Z_{j-1}-j \Delta M_{j}\right) .
$$

Since $\left(T_{1 n}-T_{5 n}\right)$ is a martingale with respect to the filtration $\left\{\mathcal{F}_{n} ; n \geq 1\right\}$, we apply the Martingale CLT (MCLT) after establishing the following conditions (see Hall and Heyde [17], Theorem 3.2):

(i) $\max _{1 \leq j \leq n}\left|\Delta S_{j n}\right| \stackrel{p}{\rightarrow} 0$;

(ii) $\sup _{n \geq 1} \boldsymbol{E}\left[\max _{1 \leq j \leq n}\left(\Delta S_{j n}\right)^{2}\right]<\infty$;

(iii) $\sum_{j=1}^{n} \boldsymbol{E}\left[\left(\Delta S_{j n}\right)^{2} \mid \mathcal{F}_{j-1}\right] \stackrel{p}{\rightarrow} \Sigma_{c}$.

For part (i), since $\left|X_{j}-Z_{j-1}\right| \leq 1$ a.s. and $\Delta M_{j}=\left(\Delta Z_{j}-\Delta A_{j}\right)$, we have that

$$
\left|\Delta S_{j n}\right| \leq n^{-1 / 2}\left(\left|X_{j}-Z_{j-1}\right|+\left|j \Delta M_{j}\right|\right) \leq n^{-1 / 2}\left(1+\left|j\left(\Delta Z_{j}-\Delta A_{j}\right)\right|\right) .
$$

Now, since $\left|\Delta Z_{j}\right|<b Y_{j-1}^{-1}$ and $\left|\Delta A_{j}\right|<c_{1} Y_{j-1}^{-2}$ a.s. by Aletti, May and Secchi [3], Lemma 2, we have

$$
\left|\Delta S_{j n}\right| \leq n^{-1 / 2}\left(1+b j Y_{j-1}^{-1}+c_{1} j Y_{j-1}^{-2}\right) \quad \text { a.s. }
$$

Since by Lemma $2.6\left(j Y_{j}^{-1}\right) \stackrel{\text { a.s. }}{\rightarrow} m^{-1}$, we have $\sup _{j \geq 1}\left(j Y_{j}^{-1}\right)<\infty$ a.s., and thus $\left|\Delta S_{j n}\right| \stackrel{\text { a.s. }}{\rightarrow} 0$. 
For part (ii), using the relation $\boldsymbol{E}[S]=\int_{0}^{\infty} \boldsymbol{P}(S>t) d t$ that holds for any non negative r.v. $S$, we obtain

$$
\boldsymbol{E}\left[\max _{1 \leq j \leq n}\left(\Delta S_{j n}\right)^{2}\right] \leq \sum_{j=1}^{n} \int_{0}^{\infty} \boldsymbol{P}\left(\left(\Delta S_{j n}\right)^{2}>t\right) d t .
$$

By applying arguments analogous to part (i), we obtain

$$
\begin{aligned}
n\left(\Delta S_{j n}\right)^{2} & \leq 2\left[\left(X_{j}-Z_{j-1}\right)^{2}+\left(j \Delta M_{j}\right)^{2}\right] \\
& \leq 2\left[1+2\left[\left(j \Delta Z_{j}\right)^{2}+\left(j \Delta A_{j}\right)^{2}\right]\right] \\
& \leq 2\left[1+2\left[b^{2}\left(j Y_{j-1}^{-1}\right)^{2}+c_{1}^{2}\left(j Y_{j-1}^{-2}\right)^{2}\right]\right] .
\end{aligned}
$$

Now, by using Markov's inequality we obtain

$$
\begin{aligned}
\boldsymbol{P}\left(\left(\Delta S_{j n}\right)^{2}>t\right) & \leq \boldsymbol{P}\left(C\left(\frac{j}{Y_{j-1}}\right)^{2}>n t\right) \\
& \leq \max \left\{1 ;\left(\frac{C}{n t}\right)^{2} \boldsymbol{E}\left[\left(\frac{j}{Y_{j-1}}\right)^{4}\right]\right\}
\end{aligned}
$$

Now, since by Aletti, May and Secchi [3], Lemma 3, $\sup _{j \geq 1} \boldsymbol{E}\left[\left(\frac{j}{Y_{j-1}}\right)^{4}\right]<\infty$, it follows that there exists a constant $C$ independent of $j$ such that $\int_{0}^{\infty} \boldsymbol{P}\left(\left(\Delta S_{j n}\right)^{2}>t\right) \leq C n^{-2}$ and hence

$$
\sup _{n \geq 1} \boldsymbol{E}\left[\max _{1 \leq j \leq n}\left(\Delta S_{j n}\right)^{2}\right] \leq \sup _{n \geq 1} C n^{-1} \leq C .
$$

For part (iii), since $\Delta M_{j}=\Delta Z_{j}-\Delta A_{j}, \Delta A_{j} \in \mathcal{F}_{j-1}$ and hence $\boldsymbol{E}\left[\Delta Z_{j} \Delta A_{j} \mid \mathcal{F}_{j-1}\right]=$ $\left(\Delta A_{j}\right)^{2}$, we have the following decomposition:

$$
\boldsymbol{E}\left[\left(\Delta S_{j n}\right)^{2} \mid \mathcal{F}_{j-1}\right]=\frac{1}{n} \boldsymbol{E}\left[Q_{j}^{2} \mid \mathcal{F}_{j-1}\right]+\frac{2}{n}\left(j \Delta A_{j}\right)^{2},
$$

where $Q_{j}:=\left(X_{j}-Z_{j-1}-j \Delta Z_{j}\right)$. Since $\left|\Delta A_{j}\right|<c_{1} Y_{j-1}^{-2}$ a.s. and by Lemma $2.6\left(j Y_{j}^{-1}\right) \stackrel{\text { a.s. }}{\rightarrow}$ $m^{-1}$, we have that $\left(j \Delta A_{j}\right)^{2} \stackrel{\text { a.s. }}{\rightarrow} 0$. Thus, $\frac{2}{n} \sum_{j=1}^{n}\left(j \Delta A_{j}\right)^{2} \stackrel{\text { a.s. }}{\rightarrow} 0$ and hence (iii) is obtained by establishing that

$$
\sum_{j=1}^{n} \boldsymbol{E}\left[\left(\Delta S_{j n}\right)^{2} \mid \mathcal{F}_{j-1}\right]=\frac{1}{n} \sum_{j=1}^{n} \boldsymbol{E}\left[Q_{j}^{2} \mid \mathcal{F}_{j-1}\right] \stackrel{p}{\rightarrow} \Sigma_{c} .
$$

To this end, we will show that $\boldsymbol{E}\left[Q_{j}^{2} \mid \mathcal{F}_{j-1}\right] \stackrel{\text { a.s. }}{\rightarrow} \Sigma_{c}$. First, note that, since $X_{j} \in\{0,1\}$, we can express $\Delta Z_{j}$ as follows

$$
\Delta Z_{j}=X_{j}\left(\left(1-Z_{j-1}\right) \frac{D_{1, j}}{Y_{j-1}}\right)+\left(1-X_{j}\right)\left(-Z_{j-1} \frac{D_{2, j}}{Y_{j-1}}\right)
$$


As a consequence, we consider $Q_{j}^{2}=X_{j} Q_{j, 1}^{2}+\left(1-X_{j}\right) Q_{j, 0}^{2}$, where, denoting by $M_{j-1}:=$ $Y_{j-1} / j$,

$$
\begin{aligned}
Q_{j, 1} & :=\left(1-Z_{j-1}\right)\left(1-\frac{D_{1, j}}{M_{j-1}}\right) \\
& =\left(\frac{1-Z_{j-1}}{M_{j-1}}\right)\left(M_{j-1}-D_{1, j}\right), \\
Q_{j, 0} & :=Z_{j-1}\left(-1+\frac{D_{2, j}}{M_{j-1}}\right) \\
& =\left(\frac{Z_{j-1}}{M_{j-1}}\right)\left(-M_{j-1}+D_{2, j}\right) .
\end{aligned}
$$

Then, since $D_{1, j}, D_{2, j}$ and $X_{j}$ are independent conditionally on $\mathcal{F}_{j-1}$ and using

$$
\begin{aligned}
\boldsymbol{E}\left[\left(M_{j-1}-D_{1, j}\right)^{2} \mid \mathcal{F}_{j-1}\right] & =\left(M_{j-1}-m\right)^{2}+\sigma_{1}^{2}, \\
\boldsymbol{E}\left[\left(-M_{j-1}+D_{2, j}\right)^{2} \mid \mathcal{F}_{j-1}\right] & =\left(M_{j-1}-m\right)^{2}+\sigma_{2}^{2},
\end{aligned}
$$

we have that

$$
\begin{aligned}
\boldsymbol{E}\left[Q_{j}^{2} \mid \mathcal{F}_{j-1}\right]= & Z_{j-1} \boldsymbol{E}\left[Q_{j, 1}^{2} \mid \mathcal{F}_{j-1}\right]+\left(1-Z_{j-1}\right) \boldsymbol{E}\left[Q_{j, 0}^{2} \mid \mathcal{F}_{j-1}\right] \\
= & Z_{j-1}\left(\frac{1-Z_{j-1}}{M_{j-1}}\right)^{2}\left[\left(M_{j-1}-m\right)^{2}+\sigma_{1}^{2}\right] \\
& +\left(1-Z_{j-1}\right)\left(\frac{Z_{j-1}}{M_{j-1}}\right)^{2}\left[\left(M_{j-1}-m\right)^{2}+\sigma_{2}^{2}\right]
\end{aligned}
$$

Finally, since by Lemma $2.6 M_{j-1} \stackrel{\text { a.s. }}{\rightarrow} m$ and by Theorem $2.4 Z_{j-1} \stackrel{\text { a.s. }}{\rightarrow} Z_{\infty}$, it follows that

$$
\sum_{j=1}^{n} \boldsymbol{E}\left[\left(\Delta \tilde{S}_{j n}\right)^{2} \mid \mathcal{F}_{j-1}\right] \stackrel{\text { a.s. }}{\rightarrow} \Sigma_{c}=Z_{\infty}\left(1-Z_{\infty}\right)\left(\frac{\bar{\Sigma}}{m^{2}}\right)
$$

For part (d), the result follows by combining part (a), (c), Crimaldi, Letta and Pratelli [10], and Crimaldi [9] and by noticing that $\left(T_{1 n}-T_{5}\right) \in \mathcal{F}_{n}$.

We now turn to consider the ARRU model. The limit distribution for an ARRU model can be obtained using Theorem 2.8 on the set of trajectories that do not cross the thresholds $\hat{\rho}_{1, n}$ and $\hat{\rho}_{2, n}$ i.o., and hence $\left\{Z_{\infty} \in\left(\rho_{2}, \rho_{1}\right)\right\}$. Since this set is not $\mathcal{F}_{n}$-measurable, we consider a sequence of sets $\left\{A_{n} ; n \geq 1\right\}$ such that $\left\{Z_{n} \in A_{n}, e v.\right\}=\left\{Z_{\infty} \in\left(\rho_{2}, \rho_{1}\right)\right\}$ a.s. Specifically, we consider the sequence of sets $\left\{A_{n} ; n \geq 1\right\}$ defined in (2.8) as follows:

$$
A_{n}:=\left(\rho_{2}+C Y_{n}^{-\alpha}, \rho_{1}-C Y_{n}^{-\alpha}\right),
$$


where $0<C<\infty$ is a positive constant and $0<\alpha<\frac{1}{2}$. Consider the partition $\Omega=\mathcal{A}_{1} \cup \mathcal{A}_{2} \cup$ $\mathcal{A}_{3}$, where

$$
\begin{aligned}
& \mathcal{A}_{1}:=\left\{Z_{k} \in A_{k}, \text { ev. }\right\} \\
& \mathcal{A}_{2}:=\left\{Z_{k} \in A_{k}, \text { i.o. }\right\} \cap\left\{Z_{k} \notin A_{k}, \text { i.o. }\right\}, \\
& \mathcal{A}_{3}:=\left\{Z_{k} \notin A_{k}, e v .\right\} .
\end{aligned}
$$

The following lemma establishes the relation between $\mathcal{A}_{j}, j \in\{1,2,3\}$, and $Z_{\infty}$.

Lemma 6.1. Assume $m_{1}=m_{2}=m$ and (2.5) with $\rho_{1}>\rho_{2}$. Then,

(a) $\mathcal{A}_{1}=\left\{Z_{\infty} \in\left(\rho_{2}, \rho_{1}\right)\right\}$ a.s.;

(b) $\boldsymbol{P}\left(\mathcal{A}_{2}\right)=0$;

(c) $\mathcal{A}_{3}=\left\{Z_{\infty} \in\left\{\rho_{2}, \rho_{1}\right\}\right\}$ a.s.

The proof of Lemma 6.1 is based on comparison arguments between the ARRU and an RRU model presented in Lemma 5.1. This relation is possible when only one random threshold modifies the dynamics of the ARRU. For this reason, we fix $\epsilon \in\left(0,\left(\rho_{1}-\rho_{2}\right) / 2\right)$ and we introduce the following times

$$
\begin{aligned}
& T_{1}:=\sup \left\{n \geq 1: Z_{n}>\min \left\{\hat{\rho}_{1 n} ; \rho_{1}-\epsilon\right\}\right\} \\
& T_{2}:=\sup \left\{n \geq 1: Z_{n}<\max \left\{\hat{\rho}_{2 n} ; \rho_{2}+\epsilon\right\}\right\}
\end{aligned}
$$

Let $\mathcal{T}_{1}:=\left\{T_{1}<\infty\right\}$ and $\mathcal{T}_{2}:=\left\{T_{2}<\infty\right\}$. Since $\hat{\rho}_{1 n}, \hat{\rho}_{2 n}$ and $Z_{n}$ converge a.s., $\boldsymbol{P}\left(\mathcal{T}_{1} \cup \mathcal{T}_{2}\right)=1$. Then, by comparing the ARRU process with the RRU process defined in (5.9) we have the following result:

Lemma 6.2. On the set $\mathcal{T}_{1}$, for any $n_{0}, k \geq 1$ we have

$$
\left\{n_{0} \geq \mathcal{T}_{1}\right\} \subset\left\{\tilde{Z}_{k}\left(n_{0}\right) \leq Z_{n_{0}+k} \leq \rho_{1}-\epsilon\right\}
$$

Analogously, on the set $\mathcal{T}_{2}$, for any $n_{0}, k \geq 1$ we have

$$
\left\{n_{0} \geq \mathcal{T}_{2}\right\} \subset\left\{\rho_{2}+\epsilon \leq Z_{n_{0}+k} \leq \tilde{Z}_{k}\left(n_{0}\right)\right\}
$$

Proof. Consider the dynamics of the RRU process $\left\{\tilde{Z}_{k}\left(n_{0}\right) ; k \geq 0\right\}$ expressed in (5.9) and the dynamics of the ARRU process $\left\{Z_{n_{0}+k} ; k \geq 0\right\}$ expressed in (5.11). Then, since $\left\{n_{0} \geq\right.$ $\left.\mathcal{T}_{1}\right\} \subset\left\{W_{1, n_{0}+k-1}=1\right\}$ and $W_{2, n_{0}+k-1} \leq 1$ we obtain (6.3). Analogously, since $\left\{n_{0} \geq \mathcal{T}_{2}\right\} \subset$ $\left\{W_{2, n_{0}+k-1}=1\right\}$ and $W_{1, n_{0}+k-1} \leq 1$ we have (6.4). 
Proof of Lemma 6.1. First, let $A:=\left[\rho_{2}, \rho_{1}\right], t_{0}=0$ and define for every $j \geq 1$

$$
\begin{aligned}
\tau_{j} & = \begin{cases}\inf \left\{k>t_{j-1}: Z_{k} \in A_{k}\right\} & \text { if }\left\{k>t_{j-1}: Z_{k} \in A_{k}\right\} \neq \varnothing ; \\
+\infty & \text { otherwise, }\end{cases} \\
t_{j} & = \begin{cases}\inf \left\{k>\tau_{j}: \tilde{Z}_{k-\tau_{j}}\left(\tau_{j}\right) \notin A\right\} & \text { if }\left\{k>\tau_{j}: \tilde{Z}_{k-\tau_{j}}\left(\tau_{j}\right) \notin A\right\} \neq \varnothing ; \\
+\infty & \text { otherwise. }\end{cases}
\end{aligned}
$$

Denoting by $T_{0}$ the last finite time in $\left\{t_{j}, \tau_{j}, j \geq 1\right\}$, we have the following partition $\Omega=S_{t} \cup$ $S_{\infty} \cup S_{\tau}$, where

$$
\begin{aligned}
S_{t} & :=\left\{T_{0} \in\left\{t_{j}, j \geq 1\right\}\right\}=\bigcap_{k \geq T_{0}}\left\{Z_{k} \notin A_{k}\right\}, \\
S_{\infty} & :=\left\{T_{0}=\infty\right\}, \\
S_{\tau} & :=\left\{T_{0} \in\left\{\tau_{j}, j \geq 1\right\}\right\}=\bigcap_{k \geq T_{0}}\left\{\tilde{Z}_{k-T_{0}}\left(T_{0}\right) \in\left(\rho_{2}, \rho_{1}\right)\right\} .
\end{aligned}
$$

Thus, we establish the following result:

(i) $\boldsymbol{P}\left(S_{\infty}\right)=0$,

(ii) $S_{\tau} \subset \mathcal{A}_{1}$, and

(iii) $S_{\tau} \subset\left\{Z_{\infty} \in\left(\rho_{2}, \rho_{1}\right)\right\}$.

For part (i), this result is obtained by establishing that there exists $i_{0} \geq 1$ such that, for any $i \geq i_{0}$,

$$
\boldsymbol{P}\left(t_{i}<\infty \mid \tau_{i}<\infty\right) \leq \frac{1}{2}
$$

To see this, we recall that by Lemma 3.4 we have, for any $h \in(0,1)$,

$$
\boldsymbol{P}\left(\sup _{k \geq 1}\left|\tilde{Z}_{k}-\tilde{Z}_{0}\right| \geq h\right) \leq \frac{b}{Y_{0}}\left(\frac{4}{h^{2}}+\frac{2}{h}\right) \leq \frac{6 b}{Y_{0}} h^{-2} .
$$

Thus, by using Lemma 3.4 with $h=C\left(\tilde{Y}_{0}\left(\tau_{j}\right)\right)^{-\alpha}$ we obtain

$$
\begin{aligned}
\boldsymbol{P}\left(t_{i}<\infty \mid \tau_{i}<\infty\right) & =\boldsymbol{P}\left(\bigcup_{k \geq 1} \tilde{Z}_{k}\left(\tau_{i}\right) \notin\left[\rho_{2}, \rho_{1}\right] \mid \tau_{i}<\infty\right) \\
& \leq \boldsymbol{P}\left(\sup _{k \geq 1}\left|\tilde{Z}_{k}\left(\tau_{j}\right)-\tilde{Z}_{0}\left(\tau_{j}\right)\right|>C\left(\tilde{Y}_{0}\left(\tau_{j}\right)\right)^{-\alpha} \mid \tau_{i}<\infty\right) \\
& \leq \boldsymbol{E}\left[\left(\frac{6 b}{\tilde{Y}_{0}\left(\tau_{j}\right)}\right)\left(C\left(\tilde{Y}_{0}\left(\tau_{j}\right)\right)^{-\alpha}\right)^{-2} \mid \tau_{i}<\infty\right] \\
& =\frac{6 b}{C^{2}} \boldsymbol{E}\left[\left(\tilde{Y}_{0}\left(\tau_{j}\right)\right)^{2 \alpha-1} \mid \tau_{i}<\infty\right],
\end{aligned}
$$


and hence the result follows by recalling that $0<\alpha<\frac{1}{2}$ and by (c) of Lemma 3.1. For part (ii), by Lemma 6.2, we have that

$$
\begin{aligned}
& \left(S_{\tau} \cap \mathcal{T}_{1}\right) \subset\left(\bigcap_{k \geq T_{0}}\left\{\tilde{Z}_{k-T_{0}}\left(T_{0}\right) \leq Z_{k} \leq \rho_{1}-\epsilon\right\}\right), \\
& \left(S_{\tau} \cap \mathcal{T}_{2}\right) \subset\left(\bigcap_{k \geq T_{0}}\left\{\rho_{2}+\epsilon \leq Z_{k} \leq \tilde{Z}_{k-T_{0}}\left(T_{0}\right)\right\}\right) .
\end{aligned}
$$

Thus, the result follows by $\boldsymbol{P}\left(\mathcal{T}_{1} \cup \mathcal{T}_{2}\right)=1$ and $\tilde{Z}_{k-T_{0}}\left(T_{0}\right) \stackrel{\text { a.s. }}{\rightarrow} \tilde{Z}_{\infty}\left(T_{0}\right) \in\left(\rho_{2}, \rho_{1}\right)$. For part (iii), from part (ii) we have that

$$
S_{\tau} \subset\left\{\min \left\{\rho_{2}+\epsilon, \tilde{Z}_{\infty}\left(T_{0}\right)\right\} \leq Z_{\infty} \leq \max \left\{\rho_{1}-\epsilon, \tilde{Z}_{\infty}\left(T_{0}\right)\right\}\right\} ;
$$

thus, the result follows by noticing that

$$
\left(\min \left\{\rho_{2}+\epsilon, \tilde{Z}_{\infty}\left(T_{0}\right)\right\}, \max \left\{\rho_{1}-\epsilon, \tilde{Z}_{\infty}\left(T_{0}\right)\right\}\right) \subset\left(\rho_{2}, \rho_{1}\right) .
$$

Now, to complete the proof of Lemma 6.1, we notice that from (i), (ii) and $\left\{\mathcal{A}_{3}=S_{t}\right\}$, it follows that $\boldsymbol{P}\left(\mathcal{A}_{2}\right)=0$ and $\left\{S_{\tau}=\mathcal{A}_{1}\right\}$. Then, combining (iii) and $\mathcal{A}_{3} \subset\left\{Z_{\infty} \in\left\{\rho_{2}, \rho_{1}\right\}\right\}$, we obtain the result.

We now present the proof of the limit distribution of the proportion of sampled ball colors for an ARRU model.

Proof of Theorem 2.10. First, take the sets $\mathcal{A}_{1}, \mathcal{A}_{2}$ and $\mathcal{A}_{3}$ defined in (6.2). Note that, since $\mathcal{A}_{1}=\underline{\lim }_{n}\left\{Z_{n} \in A_{n}\right\}$ and $\mathcal{A}_{3}^{c}=\varlimsup_{\lim }\left\{Z_{n} \in A_{n}\right\}$, by Lemma 6.1 we have

$$
\underline{\lim }_{n}\left\{Z_{n} \in A_{n}\right\}=\varlimsup_{n}\left\{Z_{n} \in A_{n}\right\}=\left\{Z_{\infty} \in\left(\rho_{2}, \rho_{1}\right)\right\} .
$$

Then, the proof is based on applying Theorem 2.8 to the ARRU model. To this end, consider the decomposition $\left\{Z_{n} \in A_{n}\right\}=\mathcal{A}_{1 n} \cup \mathcal{A}_{2 n} \cup \mathcal{A}_{3 n}$, where $\mathcal{A}_{j n}=\left\{Z_{n} \in A_{n}\right\} \cap \mathcal{A}_{j}$ for any $j \in$ $\{1,2,3\}$. Since by using Lemma 6.1 $\boldsymbol{P}\left(\mathcal{A}_{2}\right)=0$, we have $\boldsymbol{P}\left(\mathcal{A}_{2 n}\right)=0$ for any $n \geq 1$. Moreover, by definition we have that $\boldsymbol{P}\left(\mathcal{A}_{3 n}\right) \rightarrow 0$ and $\boldsymbol{P}\left(\mathcal{A}_{1 n}\right) \rightarrow \boldsymbol{P}\left(\mathcal{A}_{1}\right)$. Thus, calling $\mathcal{N}_{n}:=\sqrt{n}\left(\frac{N_{1 n}}{n}-\right.$ $Z_{\infty}$ ), we have

$$
\lim _{n \rightarrow \infty} \boldsymbol{P}\left(\mathcal{N}_{n} \leq x,\left\{Z_{n} \in A_{n}\right\}\right)=\lim _{n \rightarrow \infty} \boldsymbol{P}\left(\mathcal{N}_{n} \leq x, \mathcal{A}_{1}\right),
$$

and since by Lemma $6.1 \mathcal{A}_{1}=\left\{Z_{\infty} \in\left(\rho_{2}, \rho_{1}\right)\right\}$, this is equivalent to

$$
\lim _{n \rightarrow \infty} \boldsymbol{P}\left(\mathcal{N}_{n} \leq x,\left\{Z_{\infty} \in\left(\rho_{2}, \rho_{1}\right)\right\}\right) .
$$

Now, consider the RRU model $\left\{\tilde{Z}_{k}\left(n_{0}\right), k \geq 1\right\}$ described in (5.9) coupled with the ARRU model $\left\{Z_{n_{0}+k}, k \geq 1\right\}$. By using Lemma 5.1 , for any $n_{0} \geq 1$, we have

$$
\left(\bigcap_{k=n_{0}}^{\infty}\left\{\hat{\rho}_{2, k} \leq Z_{k} \leq \hat{\rho}_{1, k}\right\}\right) \subset\left(\bigcap_{k=1}^{\infty}\left\{Z_{n_{0}+k}=\tilde{Z}_{k}\left(n_{0}\right)\right\}\right) .
$$


Hence, on this set the ARRU process $Z_{n_{0}+k}$ is equivalent to the RRU process $\tilde{Z}_{k}\left(n_{0}\right)$; thus, we can obtain the limit distribution for the ARRU by applying the limit distribution for the RRU expressed in Theorem 2.8 on the set where the trajectories of the two processes are equivalent. To this end, define

$$
T^{*}:=\sup \left\{k \geq 1:\left\{Z_{k}<\hat{\rho}_{2, k}\right\} \cup\left\{Z_{k}>\hat{\rho}_{1, k}\right\}\right\},
$$

and note that, for any $n_{0} \geq 1$,

$$
\left\{T^{*} \leq n_{0}\right\} \subset\left(\bigcap_{k=1}^{\infty}\left\{Z_{n_{0}+k}=\tilde{Z}_{k}\left(n_{0}\right)\right\}\right) .
$$

Let $\mathcal{S}$ be a r.v. with characteristic function $\boldsymbol{E}\left[\exp \left(\frac{1}{2} \Sigma t^{2}\right)\right]$. Thus, by applying Theorem 2.8 we have that, for any $n_{0} \geq 1$ and any set $\mathcal{T} \in \mathcal{F}$,

$$
\lim _{n \rightarrow \infty} \boldsymbol{P}\left(\mathcal{N}_{n} \leq x, \mathcal{T} \cap\left\{T^{*} \leq n_{0}\right\}\right)=\boldsymbol{P}\left(\mathcal{S} \leq x, \mathcal{T} \cap\left\{T^{*} \leq n_{0}\right\}\right)
$$

Now, since $\left\{Z_{\infty} \in\left(\rho_{2}, \rho_{1}\right)\right\} \subset\left\{T^{*}<\infty\right\}$, we have

$$
\lim _{n_{0} \rightarrow \infty} \boldsymbol{P}\left(\left\{T^{*} \leq n_{0}\right\} \cap\left\{Z_{\infty} \in\left(\rho_{2}, \rho_{1}\right)\right\}\right)=\boldsymbol{P}\left(Z_{\infty} \in\left(\rho_{2}, \rho_{1}\right)\right)
$$

which implies that

$$
\lim _{n \rightarrow \infty} \boldsymbol{P}\left(\mathcal{N}_{n} \leq x,\left\{Z_{\infty} \in\left(\rho_{2}, \rho_{1}\right)\right\}\right)=\boldsymbol{P}\left(\mathcal{S} \leq x,\left\{Z_{\infty} \in\left(\rho_{2}, \rho_{1}\right)\right\}\right)
$$

This concludes the proof.

\section{Acknowledgements}

The authors would like to thank the anonymous reviewers for their valuable comments and suggestions that improved the presentation of the paper.

\section{References}

[1] Aletti, G. and Ghiglietti, A. (2017). Interacting generalized Friedman's urn systems. Stochastic Process. Appl. DOI:10.1016/j.spa.2016.12.003.

[2] Aletti, G., Ghiglietti, A. and Paganoni, A.M. (2013). Randomly reinforced urn designs with prespecified allocations. J. Appl. Probab. 50 486-498. MR3102495

[3] Aletti, G., May, C. and Secchi, P. (2009). A central limit theorem, and related results, for a two-color randomly reinforced urn. Adv. in Appl. Probab. 41 829-844. MR2571318

[4] Aletti, G., May, C. and Secchi, P. (2012). A functional equation whose unknown is $\mathcal{P}([0,1])$ valued. J. Theoret. Probab. 25 1207-1232. MR2993019

[5] Athreya, K.B. and Karlin, S. (1968). Embedding of urn schemes into continuous time Markov branching processes and related limit theorems. Ann. Math. Stat. 39 1801-1817. MR0232455 
[6] Bai, Z.D. and Hu, F. (1999). Asymptotic theorems for urn models with nonhomogeneous generating matrices. Stochastic Process. Appl. 80 87-101. MR1670107

[7] Bai, Z.D. and Hu, F. (2005). Asymptotics in randomized urn models. Ann. Appl. Probab. 15 914-940.

[8] Chen, L.H.Y. (1978). A short note on the conditional Borel-Cantelli lemma. Ann. Probab. 6 699-700. MR0496420

[9] Crimaldi, I. (2009). An almost sure conditional convergence result and an application to a generalized Pólya urn. Int. Math. Forum 4 1139-1156. MR2524635

[10] Crimaldi, I., Letta, G. and Pratelli, L. (2007). A strong form of stable convergence. In Séminaire de Probabilités XL. Lecture Notes in Math. 1899 203-225. Berlin: Springer. MR2409006

[11] Dembo, A. and Zeitouni, O. (1998). Large Deviations Techniques and Applications, 2nd ed. Applications of Mathematics (New York) 38. New York: Springer. MR1619036

[12] Durham, S.D., Flournoy, N. and Li, W. (1998). A sequential design for maximizing the probability of a favourable response. Canad. J. Statist. 26 479-495. MR1646698

[13] Durrett, R. (2010). Probability: Theory and Examples, 4th ed. Cambridge Series in Statistical and Probabilistic Mathematics 31. Cambridge: Cambridge Univ. Press. MR2722836

[14] Ghiglietti, A. and Paganoni, A.M. (2014). Statistical properties of two-color randomly reinforced urn design targeting fixed allocations. Electron. J. Stat. 8 708-737. MR3211029

[15] Ghiglietti, A. and Paganoni, A.M. (2016). An urn model to construct an efficient test procedure for response adaptive designs. Stat. Methods Appl. 25 211-226. MR3506205

[16] Ghiglietti, A., Vidyashankar, A.N. and Rosenberger, W.F. (2017). Central limit theorem for an adaptive randomly reinforced urn model. Ann. Appl. Probab. To appear.

[17] Hall, P. and Heyde, C.C. (1980). Martingale Limit Theory and Its Application. Probability and Mathematical Statistics. New York-London: Academic Press, Inc. [Harcourt Brace Jovanovich, Publishers].

[18] Hu, F. and Rosenberger, W.F. (2006). The Theory of Response-Adaptive Randomization in Clinical Trials. Wiley Series in Probability and Statistics. Hoboken, NJ: Wiley-Interscience [John Wiley \& Sons]. MR2245329

[19] Laruelle, S. and Pagès, G. (2013). Randomized urn models revisited using stochastic approximation. Ann. Appl. Probab. 23 1409-1436.

[20] Mahmoud, H. (2008). Pólya Urn Models. Boca Raton, FL: CRC press.

[21] May, C. and Flournoy, N. (2009). Asymptotics in response-adaptive designs generated by a two-color, randomly reinforced urn. Ann. Statist. 37 1058-1078. MR2502661

[22] Muliere, P., Paganoni, A.M. and Secchi, P. (2006). A randomly reinforced urn. J. Statist. Plann. Inference 136 1853-1874.

[23] Pemantle, R. and Volkov, S. (1999). Vertex-reinforced random walk on $\mathbf{Z}$ has finite range. Ann. Probab. 27 1368-1388. MR1733153

[24] Smythe, R.T. (1996). Central limit theorems for urn models. Stochastic Process. Appl. 65 115-137. MR1422883

[25] Zhang, L.-X., Hu, F. and Cheung, S.H. (2006). Asymptotic theorems of sequential estimation-adjusted urn models. Ann. Appl. Probab. 16 340-369.

Received August 2015 and revised February 2017 